\title{
THE GALACTIC POSITRON ANNIHILATION RADIATION AND THE PROPAGATION OF POSITRONS IN THE INTERSTELLAR MEDIUM
}

\author{
J. C. Higdon ${ }^{1,3}$, R. E. Lingenfelter ${ }^{2}$, And R. E. RothschiLD ${ }^{2}$ \\ ${ }^{1}$ W. M. Keck Science Center, Claremont Colleges, Claremont, CA 91711-5916, USA; jimh@ lobach.JSD.claremont.edu \\ ${ }^{2}$ Center for Astrophysics and Space Sciences, University of California San Diego, La Jolla, CA 92093, USA; rlingenfelter@ucsd.edu, rrothschild@ucsd.edu \\ Received 2007 November 19; accepted 2009 March 25; published 2009 May 20
}

\begin{abstract}
The ratio of the luminosity of diffuse $511 \mathrm{keV}$ positron annihilation radiation, measured by INTEGRAL in its four years, from a Galactic "positron bulge" $(<1.5 \mathrm{kpc})$ compared to that of the disk is $\sim 1.4$. This ratio is roughly 4 times larger than that expected simply from the stellar bulge-to-disk ratio of $\sim 0.33$ of the Galactic supernovae (SNe), which are thought to be the principal source of the annihilating positrons through the decay of radionuclei made by explosive nucleosynthesis in the SNe. This large discrepancy has prompted a search for new sources. Here, however, we show that the measured $511 \mathrm{keV}$ luminosity ratio can be fully understood in the context of a Galactic $\mathrm{SN}$ origin when the differential propagation of these $\sim \mathrm{MeV}$ positrons in the various phases of the interstellar medium is taken into consideration, since these relativistic positrons must first slow down to energies $\leqslant 10 \mathrm{eV}$ before they can annihilate. Moreover, without propagation, none of the proposed positron sources, new or old, can explain the two basic properties on the Galactic annihilation radiation: the fraction of the annihilation that occurs through positronium formation and the ratio of the broad/narrow components of the $511 \mathrm{keV}$ line. In particular, we show that in the neutral phases of the interstellar medium, which fill most of the disk ( $>3.5 \mathrm{kpc})$, the cascade of the magnetic turbulence, which scatters the positrons, is damped by ion-neutral friction, allowing positrons to stream along magnetic flux tubes. We find that nearly $1 / 2$ of the positrons produced in the disk escape from it into the halo. On the other hand, we show that within the extended, or interstellar, bulge $(<3.5 \mathrm{kpc})$, essentially all of the positrons are born in the hot plasmas which fill that volume. We find that the diffusion mean free path is long enough that only a negligible fraction annihilate there and $\sim 80 \%$ of them escape down into the $\mathrm{H}$ II and $\mathrm{H}$ I envelopes of molecular clouds that lie within $1.5 \mathrm{kpc}$ before they slow down and annihilate, while the remaining $\sim 20 \%$ escape out into the halo and the disk beyond. This propagation accounts for the low observed annihilation radiation luminosity of the disk compared to the bulge. In addition, we show that the primary annihilation sites of the propagating positrons in both the bulge and the disk are in the warm ionized phases of the interstellar medium. Such annihilation can also account for those two basic properties of the emission, the fraction $(\sim 93 \% \pm 7 \%)$ of annihilation via positronium and the ratio $(\sim 0.5)$ of broad $(\sim 5.4 \mathrm{keV})$ to narrow $(\sim 1.3 \mathrm{keV})$ components of the bulge $511 \mathrm{keV}$ line emission. Moreover, we expect that the bulk of this broad line emission comes from the tilted disk region $(0.5<R<1.5 \mathrm{kpc})$ with a very large broad/narrow flux ratio of $\sim 6$, while much of the narrow line emission comes from the inner bulge $(R<0.5 \mathrm{kpc})$ with a negligible broad/narrow flux ratio. Separate spectral analyses of the $511 \mathrm{keV}$ line emission from these two regions should be able to test this prediction, and further probe the structure of the interstellar medium. Lastly, we show that the asymmetry in the inner disk annihilation line flux, which has been suggested as added evidence for new sources, can also be fully understood from positron propagation and the asymmetry in the inner spiral arms as viewed from our solar perspective without any additional sources.
\end{abstract}

Key words: cosmic rays - elementary particles - gamma rays: theory - ISM: general - nuclear reactions, nucleosynthesis, abundances - supernovae: general

\section{INTRODUCTION}

The discovery (Johnson et al. 1972; Leventhal et al. 1979) of the diffuse Galactic positron annihilation radiation at $511 \mathrm{keV}$ from the inner Galaxy has led to extensive studies of the possible origin of the annihilating positrons. These studies have shown (e.g., Ramaty \& Lingenfelter 1979; Knödlseder et al. 2005) that, of all the various potential sources, the positrons from the decay of radioactive nuclei produced by explosive nucleosynthesis in supernovae ( $\mathrm{SNe}$ ) are the largest and, hence, most likely source.

Recent measurements, however, have raised new questions about the source. Analyses of the measurements by the gammaray spectrometer (SPI) on ESA's INTEGRAL spacecraft of the distribution of the diffuse Galactic positron annihilation radiation have shown that the luminosity of a Galactic positron

\footnotetext{
3 On Sabbatical 2008-2009 at California Institute of Technology, Pasadena, CA 91125, USA.
}

bulge within $1.5 \mathrm{kpc}$ of the Galactic center is $\sim 1.4$ times that of the Galactic disk (Knödlseder et al. 2005; Weidenspointner et al. 2007, 2008a). Their best-fit models are either concentric shell sources, or Gaussian spheroidal sources, in a bulge extending out to $\sim 1.5 \mathrm{kpc}$ combined with a thick disk with a half thickness of $z \sim 0.4 \mathrm{kpc}$, that extends from the Galactic center to well beyond the solar distance of $8 \mathrm{kpc}$. They also find a large additional contribution from a spherical halo extending beyond the bulge.

In order to compare with these observations, we use these model fitting definitions of the positron bulge, thick disk, and halo, following Knödlseder et al. (2005) and Weidenspointner et al. (2007, 2008a) in all of the discussion that follows, although both have been defined in various other ways in the literature.

\subsection{1 keV Line Bulge/Disk Luminosity Ratio}

The best-fit analysis of the SPI/INTEGRAL first year's data (Knödlseder et al. 2005, Shells+D1) gave a $511 \mathrm{keV}$ line

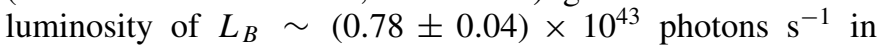


a spherical bulge from 0 to $1.5 \mathrm{kpc}$ and an old, thick disk luminosity of $L_{D} \sim(0.26 \pm 0.07) \times 10^{43}$ photons s ${ }^{-1}$, scaled to a solar distance of $R_{O} \sim 8 \mathrm{kpc}$. This gave a bulge-todisk $511 \mathrm{keV}$ luminosity ratio of $L_{B} / L_{D} \sim 3.0 \pm 0.8$. The subsequent best-fit analyses of the first two years' data, which included more coverage of the disk (Weidenspointner et al. 2007 , for four nested shells and old disk), gave a bulge flux of $\sim(0.79 \pm 0.07) \times 10^{-3}$ photons $\mathrm{cm}^{-2} \mathrm{~s}^{-1}$ in spherical shells out to $1.5 \mathrm{kpc}$, a thick disk flux of $\sim(1.41 \pm 0.35) \times 10^{-3}$ photons $\mathrm{cm}^{-2} \mathrm{~s}^{-1}$, and a marginally detected spherical halo flux of $\sim(0.86 \pm 0.59) \times 10^{-3}$ photons cm $\mathrm{cm}^{-2} \mathrm{~s}^{-1}$ from 1.5 to $8 \mathrm{kpc}$. To infer the corresponding $511 \mathrm{keV}$ line luminosities, we use the flux to luminosity ratios from their earlier modeling (Knödlseder et al. 2005, Table 3) which are essentially just $4 \pi R^{2}$ where $R \sim R_{o}$ for the bulge, about $0.57 R_{o}$ for the thick disk, and about $0.93 R_{o}$ for the halo. From the best-fit fluxes, we infer a $511 \mathrm{keV}$ positron bulge luminosity $L_{B} \sim(0.60 \pm 0.05) \times 10^{43}$ photons $\mathrm{s}^{-1}$ in the spherical shells out to $1.5 \mathrm{kpc}$, and a thick disk luminosity of $L_{D} \sim(0.36 \pm 0.09) \times 10^{43}$ photons s $^{-1}$ for a $L_{B} / L_{D} \sim 1.7 \pm 0.5$.

Most recently from more than four years of observation, Weidenspointner et al. (2008a, model BD) fit the $511 \mathrm{keV}$ fluxes and luminosities to a two-component narrow (an FWHM of $3^{\circ}$, or $0.42 \mathrm{kpc}$ ) and wide (FWHM of $11^{\circ}$, or $1.5 \mathrm{kpc}$ ) spheroidal Gaussian distribution and thick disk from more extensive observations that increased the effective sampling distance to $0.75 R_{o}$ for the disk. They found best-fit fluxes of $\sim(0.75 \pm 0.09) \times 10^{-3}$ photons $\mathrm{cm}^{-2} \mathrm{~s}^{-1}$ from the bulge and $\sim(0.94 \pm 0.16) \times 10^{-3}$ photons $\mathrm{cm}^{-2} \mathrm{~s}^{-1}$ from the disk. Scaled to $R_{o} \sim 8 \mathrm{kpc}$, these fluxes give a best-fit $511 \mathrm{keV}$ luminosity $L_{B} \sim(0.57 \pm 0.08) \times 10^{43}$ photons $\mathrm{s}^{-1}$ in the spherical bulge $<1.5 \mathrm{kpc}$, and a thick disk luminosity of $L_{D} \sim(0.40 \pm 0.06) \times 10^{43}$ photons s ${ }^{-1}$. This gives a bulgeto-disk $511 \mathrm{keV}$ luminosity ratio of $L_{B} / L_{D} \sim 1.4 \pm 0.3$. They also found a best-fit combined halo and bulge luminosity of

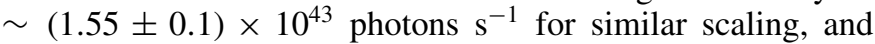
subtracting the bulge luminosity, suggests a spherical halo luminosity of $L_{H} \sim 1 \times 10^{43}$ photons s${ }^{-1}$ beyond the bulge for a total Galactic $511 \mathrm{keV}$ luminosity $L_{G} \sim(2.0 \pm 0.2) \times$ $10^{43}$ photons s $^{-1}$.

Best-fit SPI/INTEGRAL spectral analyses by Weidenspointner et al. (2008b) also show an asymmetry in the positron annihilation line flux from two opposing inner disk components at $0^{\circ}-50^{\circ}$ to either side of the Galactic bulge. These show an $80 \% \pm 40 \%$ excess in the $511 \mathrm{keV}$ line flux at the negative longitudes compared to the positive.

Assuming simply that the positron annihilation and production rates are in local equilibrium, these observations of the luminosities have been taken to imply a similar bulge/disk ratio and asymmetric spatial distribution for Galactic positron production. This suggested production ratio and distribution has challenged SN source models, since it is much larger than that expected from the distribution and mean bulge/disk ratio of Galactic SNe (e.g., Knödlseder et al. 2005; Weidenspointner et al. 2008b). As we show, however, these spatial properties can be fully explained by positron propagation.

\section{2. $511 \mathrm{keV}$ Line Width and Positronium Fraction}

We also consider the other two fundamental spectral properties of the Galactic positron annihilation: the ratio of the broad to narrow $511 \mathrm{keV}$ line emission and the fraction $\left(f_{P S}\right)$ of the annihilation that occurs via positronium (Ps) formation. As shown by Jean et al. (2006) neither of these can be explained by any of the proposed positron sources, either old or new, without extensive positron propagation, since all of these sources are expected to produce most of the positrons in the hot tenuous phases of the interstellar medium, which cannot be their primary annihilation site. For this reason alone a detailed treatment is required of the production, propagation, and annihilation in each phase and region of the Galaxy.

Studies (e.g., Guessoum et al. 1991, 2005) show that positron annihilation in different phases of the interstellar medium occurs in differing ratios of direct annihilation on both free and bound electrons to indirect annihilation via positronium formation, depending on the ionization fraction and temperature. Direct annihilation produces two photons at $511 \mathrm{keV}$, while positronium annihilation produces either two $511 \mathrm{keV}$ photons or a threephoton continuum depending on the spin state. Positronium is formed $25 \%$ of the time in the singlet state, parapositronium, which annihilates with a mean life of $\sim 1.25 \times 10^{-10}$ s into two $511 \mathrm{keV}$ photons, while $75 \%$ of the time it forms in the triplet state, orthopositronium, which annihilates with a mean life of $\sim 1.4 \times 10^{-7} \mathrm{~s}$ into a three-photon continuum between 0 and $511 \mathrm{keV}$ (e.g., Guessoum et al. 1991).

The SPI/INTEGRAL measurements of the ratio of the Galactic $2 \gamma 511$ line flux to that of the $3 \gamma$ continuum in the bulge and disk shows that the bulk of the positrons annihilate via positronium (Ps) with $f_{P s} \sim 94 \% \pm 6 \%$ (Churazov et al. 2005), $\sim 92 \% \pm 9 \%$ (Weidenspointner et al. 2006), and $95 \% \pm 3 \%$ (Jean et al. 2006). The weighted mean positronium fraction of $\sim 94 \% \pm 4 \%$ also allows us to determine the total Galactic positron annihilation rates, $A$, in the bulge and disk, since $A=\left(e^{+} / \gamma_{511}\right) \mathrm{L}$. From the above we see that $\left(e^{+} / \gamma_{511}\right)=\left[2\left(f_{P_{s}} / 4\right)+2\left(1-f_{P s}\right)\right]^{-1}$, which equals $1.69 \pm 0.17$. Thus the inferred best-fit positron bulge and Galactic disk annihilation rates are $A_{B} \sim(0.96 \pm 0.17) \times 10^{43} \mathrm{e}^{+} \mathrm{s}^{-1}$ and $A_{D} \sim$ $(0.68 \pm 0.12) \times 10^{43} \mathrm{e}^{+} \mathrm{s}^{-1}$ using the previously derived $L_{B}$ and $L_{D}$. In the hot tenuous plasma of the halo, however, the positronium fraction depends on the refractory grain abundance, ranging from only about $18 \%$ with narrow (FWHM $\sim 2 \mathrm{keV}$ ) $511 \mathrm{keV}$ line emission, if all the refractories are in grains, to $42 \%$ with broad (FWHM $\sim 11 \mathrm{keV}$ ) if the grains all disintegrated (Jean et al. 2006). Thus we assume a possible range of $\left(e^{+} / \gamma_{511}\right) \sim$ $0.65 \pm 0.07$ there. From the best-fit halo flux, we thus infer a halo annihilation rate of $A_{H} \sim(0.65 \pm 0.11) \times 10^{43} \mathrm{e}^{+} \mathrm{s}^{-1}$, implying a total Galactic positron production rate of $\sim(2.3 \pm 0.2) \times$ $10^{43} \mathrm{e}^{+} \mathrm{s}^{-1}$.

The width of the $511 \mathrm{keV}$ line also depends on the temperature of the medium and its state of ionization which effects the fraction of positronium formed by charge exchange with $\mathrm{H}, \mathrm{H}_{2}$, and $\mathrm{He}$ by superthermal, $\sim 10 \mathrm{eV}$, positrons as they slow down (Guessoum et al. 1991, 2005). The prompt annihilation of this fast moving positronium produces a characteristic broad (FWHM $\sim 6 \mathrm{keV}$ ) component of the $511 \mathrm{keV}$ line, while the subsequent thermal annihilation, either directly on free and bound electrons or via positronium formed by radiative combination or charge exchange give much narrower $(<2 \mathrm{keV})$ lines. Even the annihilation in the hot $\left(T \sim 10^{6} \mathrm{~K}\right)$, tenuous ionized medium which would produce a broader $(\sim 10 \mathrm{keV})$ line from the hot free elections, can instead produce a narrow line if most of the positrons annihilate on refractory dust grains (Guessoum et al. 1991, 2005). The dust, as it does with interstellar molecule formation, provides a two-dimensional regime with much higher interaction rates for surface chemistry.

The width of the Galactic bulge $511 \mathrm{keV}$ line measured by SPI/INTEGRAL from the first years' data has been recently 
fitted (Churazov et al. 2005; Jean et al. 2006) by two components with about $67 \% \pm 10 \%$ of the emission in a narrow line with a width of $1.3 \pm 0.4 \mathrm{keV}$ and the remaining $33 \% \pm 10 \%$ in a broad line with a width of $5.4 \pm 1.2 \mathrm{keV}$ by positronium formation in flight, giving a broad/narrow $511 \mathrm{keV}$ line flux ratio of $\sim 0.5$. The combined positronium fraction and relative fluxes of the broad and narrow $511 \mathrm{keV}$ line emission strongly constrain the bulge annihilation processes, as we discuss in detail below.

\subsection{Positron Production and Propagation}

The large difference between the ratio of the $511 \mathrm{keV}$ annihilation line bulge/disk luminosity and that of the expected positron production by $\mathrm{SNe}$ has led to suggestions that there is some new, unrecognized source of positrons in the Galactic bulge. This seems unlikely, however, since various recent reviews (e.g., Dermer \& Murphy 2001; Knödlseder et al. 2005; Guessoum et al. 2006) of the potential Galactic positron sources all conclude that $\mathrm{SNe}$ are still the most plausible source and that other suggested sources, including cosmic ray interactions, novae, and various exotic processes, all seem to be weaker and much less certain.

However, as we have shown (e.g., Guessoum et al. 1991), the $511 \mathrm{keV}$ annihilation emission only illuminates the annihilation sites, not the sources, of the positrons, since these positrons must first slow down to energies $\leqslant 10 \mathrm{eV}$ before they can annihilate. Thus, we examine in detail the propagation and annihilation of the $\beta^{+}$-decay positrons in the bulge, disk, and halo, and we show here that the expected positron propagation and annihilation are very different in the bulge and disk, and the cloud and intercloud environments. When these differences are taken into consideration, we find that the spatial distribution, the $511 \mathrm{keV}$ line widths and the positronium fraction can all in fact be clearly understood in the context of a Galactic SN origin.

Although positron propagation has previously been discussed, the arguments range from the simple suggestion (Prantzos 2006) that most of the disk positrons might diffuse along dipolar field lines and annihilate in the bulge, to Monte Carlo transport simulations (Gillard et al. 2007) suggesting that they travel less than a few hundred parsecs in all but the hot phase. We therefore also carefully examine both the physics of $\mathrm{MeV}$ electron propagation and the observational evidence of such propagation from extensive measurements of solar flare and Jovian electrons in the heliosphere.

Here, we examine in detail the mechanisms of the relativistic positron propagation in the various phases of the interstellar medium.

Overall, we assume that the Galactic stellar and interstellar distributions are defined by two superimposed systems: a stellar bulge-disk-and-halo system of stars and an interstellar bulgedisk-and-halo system of gas and plasma, as shown in Figure 1. The stellar bulge and disk populations essentially determine the distribution of SNe from the decay of whose radionuclei the positrons are born. Following Ferrière et al. (2007), the interstellar bulge, disk, and halo define the distribution of gas and plasma where the positrons die by annihilation.

As we discuss in more detail later, the stellar bulge is confined within the interstellar bulge, which is blown out by a bulge wind to about $3.5 \mathrm{kpc}$ in the Galactic plane and above the disk it feeds and merges into the halo. The stellar disk extends not only throughout the interstellar disk out to at least about $15 \mathrm{kpc}$, but also all the way into the interstellar bulge. The interstellar disk, however, extends in only to the outer edge of the interstellar bulge, and starts in the so-called "molecular ring" at around $3.5 \mathrm{kpc}$, which is defined primarily by the two opposing innermost spiral arms.

Threading the combined stellar and interstellar systems is the Galactic magnetic field, along whose flux tubes the positrons travel from their birth to their death. We assume that the flux tubes are nearly vertical (e.g., Beck 2001), perhaps dipolar, in the inner part of the interstellar bulge and are blown out by the bulge wind (e.g., Bregman 1980; Blitz et al. 1993) roughly radially in the outer bulge, out to the molecular ring, where they begin to be tightly wound into the spiral arms of the interstellar disk (e.g., Beck 2001). There, in giant star formation regions along these spiral arms, hot, massive OB star associations are formed, whose collective SNe generate superbubbles that blow out plasma as well as flux tubes into the overlying halo (e.g., Parker 1979).

Within this framework we model the various aspects of the production, propagation and annihilation of Galactic positrons.

We show that roughly half of the Galactic positrons are born in the extended interstellar bulge (e.g., Ferrière et al. 2007) in the inner $\sim 3.5 \mathrm{kpc}$ of the Galaxy, and nearly all result from SNIa occurring in the hot, tenuous high-pressure plasma fills nearly all of that volume. We show that $\sim \mathrm{MeV}$ positrons created in these SNIa are expected to escape from the remnants into the surrounding tenuous plasma. There they propagate along magnetic flux tubes by one-dimensional diffusion, resonantly scattered in pitch angle by turbulently generated magnetohydrodynamic (MHD) waves that cascade down to the thermal electron gyroradius. In these plasmas the diffusion mean free path is long enough that only a negligible fraction annihilate there and $\sim 80 \%$ of the positrons escape down into the dense $\mathrm{H}$ II and $\mathrm{H}$ I shells of neighboring molecular clouds within $1.5 \mathrm{kpc}$, where they quickly slow down and annihilate, while the remaining $\sim 20 \%$ escape out into the halo and disk beyond.

In the Galactic disk beyond $3.5 \mathrm{kpc}$, the filling factor of hot $\left(\sim 10^{6} \mathrm{~K}\right)$ plasma is more modest $(\sim 20 \%)$. Here the plasma is mostly in superbubbles, created by the correlated core-collapse SNe of massive stars. Consequently, the bulk of positron production via ${ }^{26} \mathrm{Al}$ decay, synthesized by these stars, occurs preferentially in the superbubbles. The larger bubbles blow out into the halo, sweeping the magnetic flux tubes up with them. Again the diffusion mean free paths of the positrons are such that the positrons escape along the flux tubes, either up into the halo, or down into the warm ionized envelopes, surrounding the base of the superbubble generated chimneys, where they slow down and annihilate.

In the warm $\left(\sim 10^{4} \mathrm{~K}\right)$, but essentially neutral $\mathrm{H}$ I gas, which fills the largest fraction of the disk $>3.5 \mathrm{kpc}$, we show that the cascade of magnetic turbulence, created at parsec scales, is damped by friction between the ions and neutral gas long before the cascading turbulence reaches the positron resonant gyroradii. Thus the positrons suffer much less scattering, and stream along the flux tubes with an isotropic pitch angle distribution. Therefore the positrons also mostly stream out into adjoining phases, either into the halo or into those warm ionized envelopes, separating the ubiquitous warm $\mathrm{H}$ I gas from the hot superbubbles, where they slow down and annihilate.

Therefore we find that roughly $1 / 2$ of the positrons, produced anywhere in the disk $>3.5 \mathrm{kpc}$, slow down and annihilate there, primarily in the warm ionized gas, while the rest escape from the 
disk into the halo. This escape thus accounts for the low observed annihilation radiation luminosity of the disk and explains the observed ratio of annihilation rates in the bulge and disk.

Moreover, the efficient diffusion of positrons out of the hot tenuous plasmas in both the bulge and disk, where nearly $70 \%$ are born, and into the warm ionized envelopes and cold neutral interiors of molecular clouds, where they annihilate, also explains both the observed positronium fraction and broad to narrow $511 \mathrm{keV}$ line flux ratio.

The positrons escaping into the halo from the bulge and disk also lead to the annihilation of roughly $1 / 3$ of all the positrons in the halo, even though there in no significant production there.

\subsection{Outline}

Although the processes of Galactic positron production, propagation, and annihilation are rather straightforward, all the details still make the determination of the expected $511 \mathrm{keV}$ line emission both lengthy and complicated.

We consider positron production from the decay of three separate long-lived radioisotopes, each synthesized by distinct classes of stars: the ${ }^{56} \mathrm{Ni}$ and ${ }^{44} \mathrm{Ti}$, respectively, by SNIa and SNIp from thermonuclear explosions of $\sim$ Gyr old accreting white dwarves in close binary systems, and the ${ }^{26} \mathrm{Al}$ by young (<40 Myr), massive ( $>8 M_{\odot}$ ) stars, most likely through WolfRayet (WR) winds and core-collapse SNII and SNIbc. The SNIa and SNIp from older stellar populations are known to occur in galactic bulges and disks, while WR stars, SNII and SNIbc, formed in extremely young stellar populations, occur in active, or recent, galactic star-formation sites, such as spiral arms, molecular-cloud complexes, and galactic nuclei. Thus in Section 2 we briefly re-examine the production of $\beta^{+}$-decay positrons by Galactic SNe and their spatial and temporal distribution in the light of recent observations and theory.

In Section 3 we then explore in detail the expected energy loss, scattering, and propagation processes of the relativistic positrons from these $\mathrm{SNe}$ in the different phases of the interstellar medium. We consider collisionless scattering of these positrons by small-scale fluctuations generated by MHD turbulence in the ionized interstellar phases, based on extensive studies and observations of relativistic electrons in the analogous turbulence in the interplanetary medium. Within this context we adopt a self-consistent transport model tied to field-aligned turbulence dependent on the properties of the ambient interstellar phases.

In Sections 4, 5, and 6 we determine the expected spatial distribution of positron production, propagation, slowing down and annihilation in the various phases of the positron bulge, disk, and halo. In each of these we review the fundamental properties of the interstellar medium, which constrain positron transport as well as leave signatures in the positron annihilation line emission features. Here we explore the very large differences between the interstellar properties, and the resulting positron propagation and annihilation, in the well studied local interstellar medium compared to those in the inner $\sim 1.5 \mathrm{kpc}$ of our Galaxy, where the diffuse $511 \mathrm{keV}$ emission is most intense.

Finally, in Sections 7 and 8 we give a summary and conclusions, comparing the expected $511 \mathrm{keV}$ line emission with the SPI/INTEGRAL observations. We show that the observed spatial and spectral features of the Galactic positron annihilation radiation can all be fully understood in the context of a SN radionuclide origin, when the differential propagation of these $\sim \mathrm{MeV}$ positrons in the various phases of the interstellar medium is taken into consideration.

\section{GALACTIC NUCLEOSYNTHETIC POSITRON SOURCES}

Here we briefly re-examine the production of positrons by $\beta^{+}$-decay radioactive nuclei produced by different types of Galactic SNe. We also re-examine the rates of such $\mathrm{SNe}$ in the Galaxy and the resulting spatial and temporal distribution of their production of positrons in both the Galactic bulge and disk in the light of recent observations and theory in order to determine the expected positron bulge/disk ratio of the such positron production, $Q_{B} / Q_{D}$.

\subsection{Positrons from Supernovae}

Positrons resulting from the decay chains of the longer-lived radioactive nuclei, ${ }^{56} \mathrm{Ni},{ }^{44} \mathrm{Ti},{ }^{26} \mathrm{Al}$, all produced by explosive nucleosynthesis in SNe, have long been thought (e.g., Colgate 1970; Burger et al. 1970; Clayton 1973; Ramaty \& Lingenfelter 1979; Chan \& Lingenfelter 1993; Milne et al. 1999) to be the major source of Galactic positrons. The total Galactic positron production rates from these radionuclei depend on their SN mass yields $M$, the $\mathrm{SN}$ occurrence rates $v$ and the survival fraction, $f$, of their positrons in the $\mathrm{SN}$ ejecta as it expands into the interstellar medium.

$$
\text { 2.1.1. }{ }^{56} \mathrm{Ni}
$$

The decay chain of ${ }^{56} \mathrm{Ni} \rightarrow{ }^{56} \mathrm{Co} \rightarrow{ }^{56} \mathrm{Fe}$ produces a positron only $19 \%$ of the time, since ${ }^{56} \mathrm{Ni}$ decays solely by electron capture with a mean life of 8.8 days into ${ }^{56} \mathrm{Co}$, which also decays primarily by electron capture, and just $19 \%$ by $\beta^{+}$emission into ${ }^{56} \mathrm{Fe}$ with a mean life of 111.4 days. Thus the production rate of positrons from ${ }^{56} \mathrm{Co}$ decay is $Q_{56}=0.19 f_{56} \dot{N}_{56}$, or $130 \times 10^{43} f_{56} v M_{56} \mathrm{e}^{+} \mathrm{s}^{-1}$, where $f_{56}$ is the time-integrated survival fraction of ${ }^{56} \mathrm{Co}$ decay positrons in the expanding $\mathrm{SN}$ ejecta, $\dot{N}_{56}$ is the production rate of ${ }^{56} \mathrm{Ni}$ atoms per s, $M_{56}$ is the ${ }^{56} \mathrm{Ni}$ yield in $M_{\odot}$ per $\mathrm{SN}$, and $v$ is the Galactic $\mathrm{SN}$ rate in SN per $100 \mathrm{yr}$. This Galactic rate alone would greatly exceed the observed rate of positron annihilation, if all of the positrons escaped from the SN ejecta, but most do not.

The nucleosynthetic yields of ${ }^{56} \mathrm{Ni}$, whose decay energy powers the visible light from the exploding ejecta, have been extensively calculated in all different types of SNe. This is particularly true of the cosmologically important Type Ia thermonuclear $\mathrm{SNe}$ in accreting white dwarves, which are best described by the deflagration model W7 of Nomoto et al. (1984) that gives a ${ }^{56} \mathrm{Ni}$ yield of $0.58 M_{\odot}$. The peculiar subclass of Type Ia(bg), also called Type Ip, SNe in accreting sub-Chandrasakar white dwarves are expected to produce $0.44 M_{\odot}$ of ${ }^{56} \mathrm{Ni}$ from the helium detonation model (Woosley et al. 1986).

Because of the relatively short decay mean life of ${ }^{56} \mathrm{Co}$, most of these positrons lose their energy and annihilate in the ejecta before it becomes either thin enough for them to escape or sufficiently rarefied for them to survive. Indeed, quantitative studies of the survival of positrons in the expanding SN ejecta (Chan \& Lingenfelter 1993; Milne et al. 1999) for various SN models have shown that essentially all of the positrons produced by its decay in the most frequent, but massive, core-collapse SNII slow down and annihilate unobserved in their much denser ejecta and do not escape into the interstellar medium.

In particular, Chan \& Lingenfelter (1993) have calculated $f_{56}$ of positrons from ${ }^{56} \mathrm{Co}$ decay expected for various $\mathrm{SN}$ model distributions of the ejecta density, velocity, and magnetic fields. They found that in the limit where the magnetic field is thoroughly tangled, the positron survival fraction for SNIa 
deflagration model, $f_{56}$, ranged from $0.1 \%$ to $2.5 \%$ for unmixed versus uniformly mixed ejecta. Alternatively in the limit that the magnetic field is fully combed out (e.g., Colgate et al. 1980) the SNIa $f_{56}$ was much greater, ranging from $5 \%$ to $13 \%$ for unmixed and uniformly mixed ejecta. Following this same procedure Milne et al. (1999) have further shown that a mean survival fraction $f_{56} \sim 3.5 \% \pm 2 \%$ for the deflagration model gave the best fits to SNIa light curves at late times $(>1 \mathrm{yr})$, when the positrons from ${ }^{56} \mathrm{Co}$ are the dominant source of energy. Using the deflagration model (Nomoto et al. 1984) ${ }^{56} \mathrm{Ni}$ yield of $0.58 M_{\odot}$, we calculate the expected positron production rate $Q_{56}=75 \times 10^{43} f_{56} v_{I a} \mathrm{e}^{+} \mathrm{s}^{-1}$.

$$
\text { 2.1.2. }{ }^{44} \mathrm{Ti}
$$

Chan \& Lingenfelter (1993) have shown that the positrons from ${ }^{44} \mathrm{Ti} \rightarrow{ }^{44} \mathrm{Sc} \rightarrow{ }^{44} \mathrm{Ca}$ decay, which produces a positron $95 \%$ of the time from $\beta^{+}$emission of ${ }^{44} \mathrm{Sc}$ to ${ }^{44} \mathrm{Ca}$, is a significant source of the annihilating positrons. The solar system abundance ratio of ${ }^{44} \mathrm{Ca}$ to ${ }^{56} \mathrm{Fe}$ of $1.23 \times 10^{-3}$ (e.g., Lodders 2003) requires a similar relative nucleosynthetic yield ratio of ${ }^{44} \mathrm{Ti}$ to ${ }^{56} \mathrm{Ni}$, since these radionuclei are the primary sources of ${ }^{44} \mathrm{Ca}$ and ${ }^{56} \mathrm{Fe}$ (Woosley \& Pinto 1988). The much longer ${ }^{44} \mathrm{Ti}$ decay mean life of $89 \mathrm{yr}$ would allow essentially all (>97\%) of its positrons to survive in the expanding ejecta of even the most massive SNe (Chan \& Lingenfelter 1993). Therefore the total Galactic positron production rate from ${ }^{44} \mathrm{Ti}$ decay can be scaled by the solar system abundance ratio of ${ }^{44} \mathrm{Ca}$ to ${ }^{56} \mathrm{Fe}$, assuming that half of the Galactic ${ }^{56} \mathrm{Fe}$ is produced by SNIa (e.g., Timmes et al. 1995 ) and $f_{44} \sim 1$, so that $Q_{44}=0.95 f_{44} \dot{N}_{44}$, or $1.2 \times 10^{43} v_{I a}$ $\mathrm{e}^{+} \mathrm{s}^{-1}$.

The most likely sources of these ${ }^{44} \mathrm{Ti}$ decay positrons are the peculiar SNIp SNe, typified by SN 1991bg (Filippenko et al. 1992; Turatto et al. 1996), so that we assume the same spatial distribution as SNIa. In particular, the primary source of Galactic ${ }^{44} \mathrm{Ca}$ appears to be He detonations in accreting subChandrasakar mass white dwarves, whose calculated (Woosley et al. 1986; Woosley \& Weaver 1994) ${ }^{44} \mathrm{Ti}$ yields of $M_{44} \sim$ $0.02 M_{56}$ are $\sim 16$ times the solar system $M_{44} / M_{56}$ ratio. And such models also give the best fits to the light curves of these peculiar SNe (e.g., Milne et al. 1999; Blinnikov \& Sorokina 2004; The et al. 2006). The $M_{44} / M_{56}$ ratios calculated for other types of SNe are much less than the solar system ratio. Thus we assume that the spatial distribution of the SNIp is the same as that of the SNIa and we also scale the ${ }^{44} \mathrm{Ti}$ production to that of ${ }^{56} \mathrm{Ni}$ in SNIa.

$$
\text { 2.1.3. }{ }^{26} \mathrm{Al}
$$

The additional contribution of positrons, produced $82 \%$ of the time from long-lived $\left(\tau_{26} \sim 1.04 \times 10^{6} \mathrm{yr}\right)$ decays of ${ }^{26} \mathrm{Al}$ to ${ }^{26} \mathrm{Mg}$, can be determined much more directly from the measured Galactic luminosity of the $1.809 \mathrm{MeV}$ line, which accompanies that decay. This luminosity implies a steady-state Galactic mass $M_{26} \sim 2.8 \pm 0.8 M_{\odot}$ of ${ }^{26} \mathrm{Al}$ (Diehl et al. 2006), or a positron production $Q_{26} \sim 0.82 M_{26} / 26 m_{p} \tau_{26} \sim(0.34 \pm 0.10) \times 10^{43} \mathrm{e}^{+}$ $\mathrm{s}^{-1}$. Thus they can contribute $\sim 15 \% \pm 4 \%$ of the total Galactic positron annihilation rate of $\sim(2.3 \pm 0.2) \times 10^{43} \mathrm{e}^{+} \mathrm{s}^{-1}$ inferred from the best-fit bulge, disk, and halo annihilation luminosity discussed above.

Knödlseder (1999) has shown that the ${ }^{26} \mathrm{Al} 1.809 \mathrm{MeV}$ line luminosity is strongly correlated with the distribution of massive stars in the disk, which confirms that it is produced in WR winds and/or in core-collapse SNe, SNII and SNIb/c.
With these calculated yields of ${ }^{56} \mathrm{Ni},{ }^{44} \mathrm{Ti}$ and ${ }^{26} \mathrm{Al}$ in various types of $\mathrm{SNe}$, we now estimate the total Galactic positron production rate from the decay of radionuclei synthesized in $\mathrm{SNe}$ in the bulge and disk, assuming no significant production in the halo,

$$
\begin{aligned}
Q_{B+D} & =Q_{56}+Q_{44}+Q_{26} \\
& =\left(75 f_{56} v_{I a}+1.2 v_{I a}+0.3\right) \times 10^{43} \mathrm{e}^{+} \mathrm{s}^{-1}
\end{aligned}
$$

scaled to the mean Galactic occurrence rate $v_{I a}$ of SNIa per $100 \mathrm{yr}$.

\subsection{Mean Supernova Rates in Our Galaxy}

Galactic SN occurrence rates depend on the Hubble class of the galaxies and are commonly defined (e.g., van den Bergh \& Tammann 1991; Cappellaro et al. 1999) in units of $\mathrm{SNu}$, equal to $1 \mathrm{SN}$ per $100 \mathrm{yr}$, times a factor of $h_{o}^{2} L_{\mathrm{Gal}}^{B L}$, where $h_{o}$ is the Hubble constant in units of $75 \mathrm{~km} \mathrm{~s}^{-1} \mathrm{Mpc}^{-1}$ and $L_{\mathrm{Gal}}^{B L}$ is the blue luminosity of the galaxy in units of $\left(10^{10} L_{\odot}^{B L}\right)^{-1}$. The Hubble classification of the Milky Way is Sbc (Binney \& Merrifield 1998, pages 605, 185, and 619; Kennicutt 2001). From a study of the extra-galactic observations Cappellaro et al. (1999) find that the SNIa rate is $0.21 \pm 0.08 \mathrm{SNu}$ in Sbc galaxies. Thus from the Galactic blue luminosity, $L_{\mathrm{Gal}}^{B L}$ of $(1.9 \pm 0.3) \times 10^{10} L_{\odot}$ (Freeman 1985), and this extragalactic rate for Sbc galaxies, we estimate the mean SN rate in our Galaxy for SNIa to be $0.40 \pm$ 0.16 per 100 years.

However, recent studies of the secular evolution of the Galactic bulge suggest that episodic ( $\sim \mathrm{Gyr})$ bursts of star formation could cause modest variations in the SN bulge/ disk ratio. From observations of oxygen-rich, cool giants, Sjouwerman et al. (1998) conclude that the most recent burst of star formation in the inner bulge occurred roughly $1 \mathrm{Gyr}$ ago and produced $\sim 10^{6} \mathrm{SNII}$ and $\mathrm{SNIb} / \mathrm{c}$. Such a burst would then be followed by a much more extended period of enhanced SNIa and SNIp, occurring with a range of delay times between 0.1 and $1 \mathrm{Gyr}$, calculated from evolutionary models by Greggio (2005). Thus, although the SNII and SNIbc all occurred during the burst of star formation a Gyr ago, we would expect the SNIa and SNIp to still be occurring at the present time. Using the average ratio of SNIa to SNII for our Galaxy of 0.25 (e.g., van den Bergh \& Tammann 1991; Cappellaro et al. 1999), we would expect $2.5 \times 10^{5}$ SNIa from this star burst spread over $\sim 1 \mathrm{Gyr}$, or an added contribution of 0.025 SNIa per 100 years. Thus we estimate the present SN rate in our Galaxy for SNIa to be 0.43 \pm 0.16 per 100 years.

Using this SNIa rate $v_{I a}$ we then estimate from Equation (1) the expected mean Galactic positron production from the decay of SN produced ${ }^{56} \mathrm{Co},{ }^{44} \mathrm{Ti}$, and ${ }^{26} \mathrm{Al}$,

$$
\begin{aligned}
Q_{B+D}= & {\left[(32 \pm 13) f_{56}+(0.5 \pm 0.2)\right.} \\
& +(0.3 \pm 0.1)] \times 10^{43} \mathrm{e}^{+} \mathrm{s}^{-1} .
\end{aligned}
$$

Thus, of the Galactic positron production of $\sim(2.3 \pm 0.2) \times$ $10^{43} \mathrm{e}^{+} \mathrm{s}^{-1}$, inferred from the observed bulge, disk, and halo annihilation radiation as discussed above, ${ }^{26} \mathrm{Al}$ decay accounts for $\sim 0.3 \times 10^{43} \mathrm{e}^{+} \mathrm{s}^{-1}$, and ${ }^{44} \mathrm{Ti}$ decay produces $\sim 0.5 \times$ $10^{43} \mathrm{e}^{+} \mathrm{s}^{-1}$, nearly all from SNIp, leaving $\sim(1.5 \pm 0.3) \times$ $10^{43} \mathrm{e}^{+} \mathrm{s}^{-1}$, which can come from ${ }^{56} \mathrm{Ni}$ decay positrons, if they have a survival fraction in SNIa ejecta of $f_{56} \sim 5 \% \pm 2 \%$, including the uncertainty in the Galactic SNIa rate. A survival fraction of $\sim 5 \%$, was in fact just what was calculated (Chan \& Lingenfelter 1993, Figure 3) for the standard deflagration model 
of SNIa (Nomoto et al. 1984 W7) with a combed out magnetic field in the ejecta. It is also quite consistent with the mean value of $\sim 3.5 \% \pm 2 \%$ inferred (Milne et al. 1999) from SNIa light curves at late times, when the positrons become a major source of ejecta heating.

\subsection{Spatial Distribution of Galactic Supernovae}

Here we investigate both the stellar bulge/disk ratio $\left(B^{*} / D^{*} \sim\left(v_{B} / v_{D}\right)_{I a+I p}\right)$ of Galactic SNe and their radial and transverse distributions within the stellar bulge and disk. Of primary concern are the SNIa and SNIp, which appear to be the source of $\sim 85 \%$ of the annihilating positrons.

We first determine the relative contributions of SNIa occurring in the Galactic stellar bulge and disk. It is well known that the SNIa occurrence rate is essentially the same across the Hubble sequence of galaxy types from ellipticals (E) through late-type spirals $(\mathrm{Sd})$, when such rates are defined in $\mathrm{SNu}$, the number of SNe per year per unit of blue luminosity of the parent galaxy (e.g., Cappellaro et al. 1999). Across this broad Hubble sequence, the fraction of galactic blue-band luminosities contributed by Galactic disks range from zero in ellipticals to $95 \%$ in late-type Scd galaxies (Simien \& de Vaucouleurs 1986), yet the SNIa birth rate per unit blue luminosity of parent galaxies remains the same.

Since the SNIa occurrence rates per blue luminosity is independent of the relative contributions of galactic bulges and disks, we scale the ratio of SNIa birthrates in the stellar bulge and disk of our Galaxy by the corresponding ratio of stellar bulge-to-disk blue luminosities, $L_{B}^{B L} / L_{D}^{B L}$. In their classic study Simien \& de Vaucouleurs (1986) investigated the systematics of bulge-to-disk ratios in the blue-band. The mean value of their tabulations of $L_{B}^{B L} / L_{D}^{B L}$ for our Galaxy is 0.25 . Thus, we expect that the Galactic stellar luminosity bulge/disk ratio implies a similar time-averaged, mean stellar bulge/disk ratio $\left(v_{B} / v_{D}\right)_{I a} \sim 0.25$ for Galactic SNIa and SNIp. Adding the star burst contribution of 0.025 SNIa per 100 years in the bulge, which is a $30 \%$ enhancement over the mean bulge rate of 0.08 SNIa per 100 years, gives a current stellar Galactic bulge/disk ratio $\left(v_{B} / v_{D}\right)_{I a+I p} \sim 0.25 \times 1.3 \sim 0.33$ for SNIa and SNIp.

To model the spatial distribution of SNIa in the Galaxy, we use the representations of Dehnen \& Binney (1998) who parameterized the large-scale stellar distributions within the Galaxy by comparing models to the Galactic rotational and other observational constraints. Thus the number density of SNIa in the Galactic disk, $n_{D *}(R, z)_{I a}$, and the number density of SNIa in the Galactic bulge, $n_{B *}(R, z)_{I a}$, expressed in cylindrically symmetric Galactocentric coordinates, are

$$
\begin{aligned}
& n_{D *}(R, z)_{I a}=n_{o_{D}} e^{-R / R_{d}-|z| / z_{d}}, \\
& n_{B *}(R, z)_{I a}=\frac{n_{o_{B}}}{\eta^{1.8}} e^{-\frac{\eta^{2}}{r_{t}^{2}}},
\end{aligned}
$$

where $\eta=\sqrt{R^{2}+(z / 0.6)^{2}}, R$ represents the planar distance from the Galactic center in kpc, $z$ represents the distance normal to the Galactic plane in $\mathrm{kpc}$, and the constant $r_{t}=1.9 \mathrm{kpc}$ (Dehnen \& Binney 1998). Here the normalization constants, $n_{O_{D}}$ and $n_{o_{B}}$ are found from the total Galactic SNIa birthrate and the $\left(v_{B} / v_{D}\right)_{I a}$ ratio. Dehnen \& Binney (1998) ascertained that the more than $90 \%$ of the stellar disk population could be represented by $z_{d}$ of $0.180 \mathrm{kpc}$. They concluded that the most important model parameter was the ratio of the disk scale length, $R_{d}$, to $R_{\odot}$, the distance of the Sun from the Galactic center. They found that the observational constraints were best satisfied by any of four models with $R_{\odot}=8 \mathrm{kpc}$ and $R_{d} / R_{\odot}$ of $0.25,0.3$, $0.35, \& 0.4$. Here we will use their second model with $R_{d}$ of $2.4 \mathrm{kpc}$. We also include a star burst contribution to the bulge located in the central molecular zone (CMZ; Sjouwerman et al. 1998).

\subsection{Spatial Distribution of Galactic Positron Production}

In Figure 2 we show the relative spatial distributions, $F(<r)$, as functions of distance, $r$, from the Galactic center for SNIa residing in the stellar bulge and disk employing the relative number densities, Equation (3) for $n_{B *}(R, z)_{I a}$ and $n_{D *}(R, z)_{I a}$, expressed a functions of $R \& z$ in a Galactocentric cylindrical coordinate system,

$$
\begin{gathered}
F_{B *}(<r)=2 \pi \int_{0}^{r} n_{B *}(R(r), z(r))_{I a} r^{2} d r, \\
F_{D *}(<r)=2 \pi \int_{0}^{r} n_{D *}(R(r), z(r))_{I a} r^{2} d r, \\
F_{O B *}(<r)=2 \pi \int_{0}^{r} n_{O B *}(R(r), z(r))_{I I \& I b c} r^{2} d r .
\end{gathered}
$$

$F_{O B *}(<r)$ is the relative spatial distribution of massive stars, the expected WR and SNII \& SNIbc progenitor sources of ${ }^{26} \mathrm{Al}$. Although the great majority of massive stars, which synthesize ${ }^{26} \mathrm{Al}$, are located in the outer $(>3.5 \mathrm{kpc})$ Galactic disk (McKee $\&$ Williams 1997), some massive stars found in the inner $(<0.2 \mathrm{kpc})$ stellar bulge. Since Galactic Lyman continuum radiation is also produced by a massive star population (e.g., McKee \& Williams 1997), we use the ratio of Lyman continuum radiation emitted by the inner stellar bulge to that from the outer disk to estimate the corresponding ${ }^{26} \mathrm{Al}$ ratio. Güsten (1989) estimated a luminosity of ionizing photons from massive stars in the inner bulge of $\sim(1.5 \pm 0.5) \times 10^{52}$ photons $\mathrm{s}^{-1}$. Comparing this to the estimated (McKee \& Williams 1997) total Galactic value of $\sim 1.9 \times 10^{53}$ photons s$^{-1}$, we expect that roughly $10 \%$ of the massive stars and their generated ${ }^{26} \mathrm{Al}$ occur in the inner bulge, amounting to $\sim 0.03 \times 10^{43} \mathrm{e}^{+} \mathrm{s}^{-1}$.

For the remaining disk portion we use the spatial distribution of OB associations, $n_{O B}(R, z)_{I I \& I b c}$, of (McKee \& Williams (1997, Equation (35)) with $z_{d}=0.15 \mathrm{kpc}$ to represent that of the ${ }^{26} \mathrm{Al}$ made by massive stars. These spatial distributions have been normalized at large $r$ to their expected relative contributions to the total Galactic positron production rate fractions: $F_{B *}=$ $0.21, F_{D *}=0.64$, and $F_{O B}=0.15$, based on the SNIa and SNIp bulge/disk ratio of 0.33 in the $85 \%$ of positrons produced by them.

As we see from Figure 2, of the positrons produced from SNIa and SNIp in the disk, $13 \%$ are born within $1.5 \mathrm{kpc}$. Although the best-fit disk model used in the SPI/INTEGRAL analyses (Weidenspointner et al. 2007, 2008a) of the annihilation radiation also includes $12 \%$ occurring within that radius, in order to treat the propagation of the positrons before they annihilate, we obviously need to include them in the total production within $1.5 \mathrm{kpc}$. Once we have determined the distribution of the subsequent annihilation of all of the positrons, we then adjust the estimated bulge $(<1.5 \mathrm{kpc})$ and disk $(>1.5 \mathrm{kpc})$ annihilation luminosities to compare with the SPI/INTEGRAL bulge and disk components, as we discuss in Section 7.

Therefore, the positron bulge production $(<1.5 \mathrm{kpc}), Q_{B} \sim$ $\left[F_{B *}+F_{D *}(<1.5)+F_{O B}(<1.5)\right] Q_{B+D} \sim[0.21+0.64(0.13)+$ $0.1(0.15)] Q_{B+D} \sim 0.31 Q_{B+D}$, and the disk production, including the $\mathrm{OB}$ contribution, $Q_{D} \sim\left[F_{D *}\left(1-F_{D *}(<1.5)\right)+\right.$ 
$\left.0.9 F_{O B}\right] Q_{B+D} \sim[0.64(0.87)+0.9(0.15)] Q_{B+D} \sim 0.69 Q_{B+D}$. Thus the effective positron production ratio in the bulge and disk within and beyond $1.5 \mathrm{kpc}$ is $\left(Q_{B} / Q_{D}\right) \sim 0.31 / 0.69 \sim 0.45$.

From the ratio of positron production rates in the bulge $Q_{B}$ and disk $Q_{D}$ we now determine the differential propagation of the positrons within and between those regions and the halo before they annihilate. In simplest terms we define the resulting positron bulge/disk annihilation ratio as, $A_{B} / A_{D} \sim$ $\left(P_{B: B} Q_{B}+P_{D: B} Q_{D}\right) /\left(P_{B: D} Q_{B}+P_{D: D} Q_{D}\right)$, where $P_{B: B}$ and $P_{D: B}$ are respectively the fractions of the positrons, born in the bulge $B$ and disk $D$ beyond $1.5 \mathrm{kpc}$, which annihilate in the positron bulge $B<1.5 \mathrm{kpc}$, while $P_{B: D}$, and $P_{D: D}$ are the corresponding fractions of those positrons which annihilate in the disk $D$ beyond the positron bulge $>1.5 \mathrm{kpc}$. As we discuss below, however, we need to investigate the positron propagation and annihilation in each of the interstellar gas phases within each of the regions.

\section{NATURE OF GALACTIC POSITRON PROPAGATION}

We now look in detail at the nature of these positrons, and their propagation, slowing down and annihilation in the various phases of the interstellar medium in the bulge, disk, and halo. In particular, we investigate the plasma processes that determine the rate of positron propagation, drawing upon the extensive studies and observations of relativistic electron propagation in the interplanetary medium.

\subsection{Relativistic $\beta^{+}$-Decay Positrons from Supernovae}

Assuming that the dominant source of Galactic positrons is the $\beta^{+}$decay of ${ }^{56} \mathrm{Co},{ }^{44} \mathrm{Sc}$ and ${ }^{26} \mathrm{Al}$ from $\mathrm{SNe}$, the detailed studies of their survival in and escape from the ejecta show that the great bulk of the surviving and escaping positrons are relativistic.

Positrons emitted in the ${ }^{56} \mathrm{Co} \rightarrow{ }^{56} \mathrm{Fe}$ decay are distributed in kinetic energy with a maximum of $1.459 \mathrm{MeV}$ and a mean of $0.630 \mathrm{MeV}$, and those from ${ }^{44} \mathrm{Sc} \rightarrow{ }^{44} \mathrm{Ca}$ decay have a very similar spectrum. The expected (Chan \& Lingenfelter 1993; Milne et al. 1999) mean energy of the escaping positrons from the shorter-lived (111.4 day mean life) decay of ${ }^{56} \mathrm{Co}$ is close to $0.5 \mathrm{MeV}$, reduced from their initial energy by ionization losses in the still dense ejecta, while the mean energy of those from the longer lived ( $89 \mathrm{yr}{ }^{44} \mathrm{Ti}$ dominated meanlife) decay of ${ }^{44} \mathrm{Sc}$ is essentially unchanged at about $0.6 \mathrm{MeV}$ in the much less dense ejecta. The mean energy of positrons from the decay of the very long-lived $\left(1.04 \times 10^{6} \mathrm{yr}\right)$ decay of ${ }^{26} \mathrm{Al}$ is likewise about $0.5 \mathrm{MeV}$.

In order for these surviving relativistic positrons to annihilate in the interstellar medium they must first be decelerated to energies, $\leqslant 10 \mathrm{eV}$. In order to slow down to thermal energies, these $\sim 0.5 \mathrm{MeV}$ positrons must run through $\sim 0.12 \mathrm{~g} \mathrm{~cm}^{-2}$ (Berger \& Seltzer 1964), or $n d_{\mathrm{sd}} \sim 6 \times 10^{22} \mathrm{H} \mathrm{cm}^{-2}$, in unionized interstellar gas, and this range is reduced by a factor of $1 /\left(1+x_{e}\right)$ if the gas is ionized with an ionization fraction, $x_{e}$, relying solely on collisional ionization losses. In the process of traveling this slowing down distance, $d_{\mathrm{sd}} \sim 20 / n\left(1+x_{e}\right)$ $\mathrm{kpc}$, the average velocity of these positrons, $\bar{\beta} c$ is $\sim 0.7$ of the velocity of light, so their slowing down time,

$$
t_{\mathrm{sd}} \sim d_{\mathrm{sd}} / \bar{\beta} c \sim 9 \times 10^{4} / n\left(1+x_{e}\right) \mathrm{yr},
$$

where $n$ is the mean $\mathrm{H}$ density in $\mathrm{cm}^{-3}$.

Thus we see that since the slowing down distance of the radioactive decay positrons in the nominal phases of the interstel- lar medium is $>0.5 \mathrm{kpc}$ in H I clouds with typical $n \sim 40 \mathrm{~cm}^{-3}$, $>50 \mathrm{kpc}$ in warm media with typical $n \sim 0.4 \mathrm{~cm}^{-3}$, and $>3 \mathrm{Mpc}$ in hot phases with typical $n \sim 3 \times 10^{-3} \mathrm{~cm}^{-3}$. Since all of these distances are far greater than the scales of their corresponding interstellar components, these fast positrons, streaming along the large-scale interstellar magnetic field, would all escape the Galactic disk before they even slow down, unless there is sufficient plasma turbulence to efficiently isotropize their trajectories. We thus investigate in detail under what conditions the interstellar turbulence can isotropize the trajectories of these relativistic positrons.

\subsection{Nature of the Turbulence which Scatters Interstellar Positrons}

In ionized interstellar phases we expect that the interstellar $\mathrm{MeV}$ positrons are scattered by resonant interactions with ambient turbulent magnetic fluctuations. Consequently, the efficiency of these scatterings in turn depends on the fundamental properties of the MHD turbulence at very small spatial scales of the electron gyroradius, $r_{e}$. However, the basic properties of MHD turbulence is poorly understood. In a recent review of turbulence and magnetic fields in astrophysical plasmas, Schekochihin \& Cowley (2007) state "despite over fifty years of research and many major advances a satisfactory theory of MHD turbulence remains elusive. Indeed, even the simplest (most idealized) cases are not fully understood."

An important advancement was the realization that in many astrophysical sites MHD turbulence is anisotropic. Such anisotropy is a well observed phenomenon in solar wind plasmas (e.g., Belcher \& Davis 1971; Matthaeus et al. 1990; Osman \& Horbury 2007) as well as in increasingly more accurate, numerical simulations (Shebalin et al. 1983; Maron \& Goldreich 2001; Cho et al. 2002; Mason et al. 2006; Perez \& Boldyrev 2008). In a pioneering study Goldreich \& Sridhar (1995) developed a sophisticated theory of anisotropic incompressible MHD turbulence. They presented the concept of "critical balance" which predicted in inertial regime of turbulent MHD flows, first, a filamentary shape for turbulent fluctuations, via a relationship between the wave numbers parallel $(\|)$ and transverse $(\perp)$ to the direction of the local mean magnetic field, $\mathrm{k}_{\|} \propto \mathrm{k}_{\perp}^{2 / 3}$ and, second, the modeled energy spectra of transverse turbulent components possesses Kolmogorov (1941) scaling, $k_{\perp}^{-5 / 3}$, observed in low-speed, terrestrial flows.

At present, a puzzling dichotomy exists between these model predictions and a plethora of solar wind observations and numerical simulations. Power spectra of small-scale electromagnetic fluctuations in solar wind plasmas have been found to have the expected $-5 / 3$ spectral index (e.g., Horbury et al. 1996; Leamon et al. 1998; Bale et al. 2005), but corresponding spectra in many precise numerical simulations have spectral indices much closer to $-3 / 2$ (Maron \& Goldreich 2001; Beresnyak \& Lazarian 2006; Mininni \& Pouquet 2007; Perez \& Boldyrev 2008). Yet the critical balance prediction about the wavenumber relationship of turbulent eddies seems to be consistent with many numerical simulations (Cho \& Vishniac 2000; Maron \& Goldreich 2001; Cho et al. 2002; Beresnyak \& Lazarian 2006). Unfortunately, analyses of single spacecraft measurements are unable to determine the full modal three-dimensional wavevector spectra of turbulent fluctuations (e.g., Horbury et al. 2005) and thus cannot confirm or refute the critical balance prediction about filamentary-shaped fluctuations (Beresnyak \& Lazarian 2008). 
Although much progress has been made in the understanding and modeling of anisotropic MHD turbulence, such models, which assume that the frequency of the turbulent fluctuations is low compared to the proton gyrofrequency $\Omega_{p}$, still have difficulty in representing high-frequency collisionless turbulence evolving on frequencies, $\omega \approx \Omega_{e}$, which seem to be most efficient at resonantly scattering $\sim \mathrm{MeV}$ positrons, where $\Omega_{e}$ represents electron gyrofrequency. The application of such models of anisotropic turbulence seems to imply that collisionless scattering of $\mathrm{MeV}$ electrons would be modest and $\mathrm{MeV}$ electron transport should be dominated by streaming at $\sim \bar{\beta} c$. Yet a great variety of independent investigations of $\mathrm{MeV}$ electron transport in the interplanetary medium irrefutably demonstrate that such particles propagate diffusively in the collisionless solar wind plasma, although the nature of the plasma fluctuations which actually scatter such electrons remains yet unknown. Thus, we employ a simple phenomenological model of collisionless $\mathrm{MeV}$ electron scarttering by turbulent interstellar fluctuations tied heavily to the well determined properties of electron scattering in turbulent solar wind plasmas.

The propagation of $\mathrm{MeV}$ electrons has been well studied in the interplanetary medium employing measurements of electrons accelerated by solar particle events as well as by the Jovian magnetosphere. The propagation of Jovian $\mathrm{MeV}$ electrons is, for the most part, dominated by diffusion (Ferreira et al. 2001a, 2001b, 2003). Fits to intensity and anisotropy time profiles of solar flare electrons also illustrate the dominance of diffusive transport at these electron energies, and, consequently, lead to the straightforward determination of scattering mean free paths via fits to intensity and anisotropy time profiles (Kallenrode 1993).

Bieber et al. (1996) related such phenomenologically derived scattering mean free paths from solar flare particle measurements to the determination of simultaneous power spectra of magnetic fluctuations at MHD spatial scales. Since it is impossible to determine the full three-dimensional wavevector spectra of turbulent fluctuation from single spacecraft measurements (e.g., Fredricks \& Coroniti 1976), Bieber, Wanner, \& Matthaeus employed a simple two-component representation of the anisotropic interplanetary MHD turbulence (Bieber et al. 1996). They found that their magnetic-field data consisted of two anisotropic populations, fluctuations with large correlation lengths transverse to the direction of the mean magnetic field (slab turbulence) and fluctuations with large correlation lengths parallel to the direction of the mean magnetic field (quasi-twodimensional turbulence). In such slab models the wave vectors of the fluctuations are aligned parallel to the direction of the mean magnetic field, and a simple interpretation is that in the inertial range slab turbulence consists of Alfvén wave propagating along the mean magnetic field. Later investigations (Oughton et al. 2004, 2006; Oughton \& Matthaeus 2005) have demonstrated that this simple two-component model of anisotropic turbulence seems to model the basic properties of solar wind MHD turbulence.

Bieber et al. (1996) found that interplanetary turbulence is dominated ${ }^{4}$ by quasi-two-dimensional turbulence, and the mean ratio of the power in slab turbulence to the power in

\footnotetext{
4 Recently, Horbury et al. (2005) have investigated in more detail the properties of anisotropic solar wind turbulence, implementing a wavelet-based method to track the direction of the local mean magnetic field; such an approach reduces greatly the noise in the magnetic field measurements. Employing the two-component model, they ascertained that their results agreed "remarkably well" with the results of Bieber et al.
}

two-dimensional turbulence is $\sim 0.15$. Further, observing that the modeled two-dimensional fluctuations do not contribute to particle scatterings, they calculated scattering mean free paths expected by resonant interactions solely with turbulent slab fluctuations via quasi-linear theory (Jokipii 1969). They found that such scattering mean free paths agreed well with the values determined independently (of the analyses of the turbulent magnetic variations) from data fits to intensity and anisotropy time profiles.

Based on these discussions, we model interstellar MHD turbulence as composite slab/two-dimensional turbulence. We assume here that the ratio of the power of interstellar slab turbulence along the mean magnetic field to the power of twodimensional turbulence normal to the direction of the mean field is 0.15 following Bieber et al. (1996). Similarly, we assume that the spectrum of interstellar slab turbulence steepens from a spectral index of $-5 / 3$ to -3 at a wavenumber $k_{d}>1 / r_{p}$, where $r_{p}$ is the proton gyroradius, since Leamon et al. (1998) have found that at higher wave numbers the spectral index of solar wind variations varied from -2 to -4.4 with an average index of -3 .

To quantify such scattering mean free paths for interstellar $\mathrm{MeV}$ positrons we use an analytic approximation (Teufel \& Schlickeiser 2002) calculated for slab MHD turbulence employing quasi-linear theory. Following Teufel \& Schlickeiser, we assume that energy is injected into the interstellar medium at an outer spatial scale, $l_{o}$. For $k l_{o} \geqslant 1$ and $k \leqslant k_{d}$, the modeled turbulence possesses a spectral index, $s$. From the discussion above, we assume $s=5 / 3$ and at $k_{d}=1 / r_{p}$, we assume the spectral shape steepens to $s=3$. Teufel \& Schlickeiser (2002, their Equation (58)) find that for such slab turbulence the electron scattering mean free path along the magnetic field is

$$
\begin{array}{r}
\lambda_{\|}=\frac{9}{2}\left(B_{o} / \delta B_{\|}\right)^{2} \frac{J^{2}}{k_{\min } a} K(a, h, s, I, J), \\
k_{\min }=\frac{2 \pi}{l_{o}}, k_{d}=\frac{1}{r_{p}}, J=\frac{\bar{\beta} c k_{\min }}{\Omega_{e}}, I=\frac{\bar{\beta} c k_{d}}{\Omega_{e}}, a=\frac{\bar{\beta} c}{V_{a}}, \\
f_{1}(s, h)=\frac{2}{h-2}+\frac{2}{2-s}, \\
J \ll I \ll 1 \ll a \\
K=\frac{a^{2}}{f_{1} J^{s} I^{2-s}}\left\{-\frac{1}{3}{ }_{2} F_{1}\left(1, \frac{3}{h-1}, \frac{h+2}{h-1},-\frac{\pi a}{f_{1}} I^{h-2}\right)\right. \\
J \ll 1 \ll I \ll a \\
K=\frac{1}{\pi}\left[\frac{1}{2-s}-\frac{1}{4-s}\right]+\frac{a^{2}}{f_{1} J^{s} I^{3-s}}{ }_{2} F_{1}\left(1, \frac{1}{h-1}, \frac{p}{h-1},-\frac{\pi a}{f_{1} I}\right)
\end{array}
$$

where $\delta B_{\|}^{2} / 8 \pi$ is the energy density of the turbulent magnetic fluctuation in the direction of the mean magnetic field, $c$ is the speed of light, $\Omega_{e}$ is the gyro-frequency of the positron, the mean magnetic field is $B_{o}, V_{a}$ is the Alfvén speed, $B_{o} / \sqrt{4 \pi \rho}, \rho$ is the ion mass density, $r_{e}$ is the electron gyroradius; the average positron speed is $\bar{\beta} c$, and $K$ is a dimensionless quantity involving a Gauss hypergeometric function ${ }_{2} F_{1}$ (Table 3 of Teufel \& Schlickeiser 2002).

Thus, by such one-dimensional diffusion the positrons in their slowing down time $t_{\text {sd }}$ would be distributed along a flux tube a mean length $l_{\mathrm{sd}} \sim\left(2 t_{\mathrm{sd}} \lambda_{\|} \bar{\beta} c / 3\right)^{1 / 2}$ in either direction from their point of origin.

\subsection{Ion-Neutral Damping}

The bulk of the Galactic interstellar mass resides in primarily neutral phases $\left(\mathrm{H}_{\mathrm{I}} \& \mathrm{H}_{2}\right)$, concentrated in a cloud population 


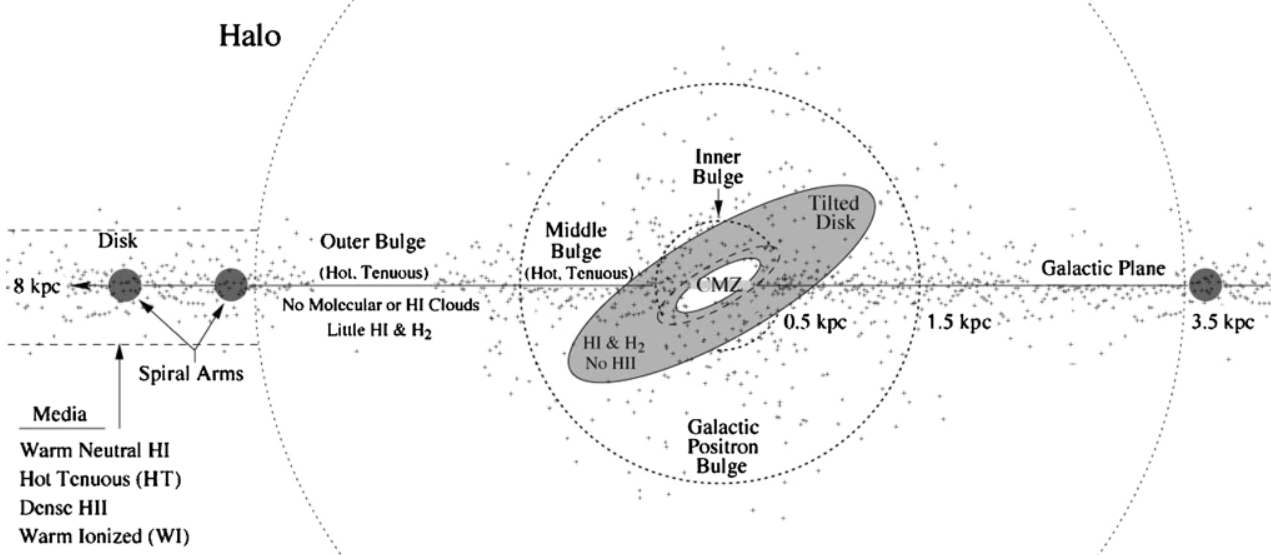

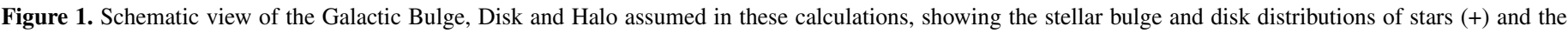
assumed boundaries of the interstellar gas and plasma subdivisions, chosen for comparison with the SPI/INTEGRAL observational analyses.

(e.g., Tielens 2005). Moreover, a major fraction of the disk $(>3.5 \mathrm{kpc}$ ) is filled with warm neutral H I (Kulkarni \& Heiles 1987). Consequently, the nature of MHD turbulence in predominantly neutral interstellar phases needs to be addressed.

In partially neutral plasmas, where magnetic forces act directly on charges, and indirectly on neutral atoms via ionneutral collisions, the turbulent NHD fluctuations are dissipated into heat when ion and neutral velocities differ significantly (Higdon 1984; Goldreich \& Sridhar 1995; Lithwick \& Goldreich 2001). Turbulent MHD cascades are quenched at scales of roughly the collision mean free path of protons with neutral atoms, if the proton collision rate with neutral atoms exceeds the eddy cascade rate. The mean free path for proton- $\mathrm{H}$ I collisions is $l_{p H}=5 \times 10^{13} / n \mathrm{~cm}$ at $8000 \mathrm{~K}$, or if hydrogen is fully ionized, and $\mathrm{He}$ is the dominant neutral species, the mean free path for proton-He collisions is $l_{p H e} \sim 1.5 \times 10^{15} / n \mathrm{~cm}$ where $n$ is the $\mathrm{H}$ number density, including both ions and neutrals (Lithwick \& Goldreich 2001).

Consequently, they showed that MHD turbulence can cascade to small spatial scales, much less than these damping collision mean free paths, only if the $\mathrm{HI}_{\mathrm{I}}$ fraction, $n_{\mathrm{HI}} / n$, is less than a critical value,

$$
n_{\mathrm{HI}} / n<f_{\text {crit }} \approx 5\left(l_{p H} / l_{o}\right)^{1 / 3} .
$$

Therefore in predominantly neutral interstellar phases turbulent MHD cascades are halted by ion-neutral collisions at $\sim l_{p H}$, spatial scales far greater than the gyroradii of $\mathrm{MeV}$ positrons. Hence we expect collisionless scattering of positrons by such large-scale turbulent MHD fluctuations to be very inefficient. Similarly simple elastic collisions with ambient electrons, whose mean pitch angle scattering is $90^{\circ}$ and mean energy loss is 50\%, have a mean free path much longer than slowing down distance due to ionization losses (e.g., Berger \& Seltzer 1964), so they too offer no significant scattering.

In the absence of such scatterings, $\sim \mathrm{MeV}$ positrons might be expected to generate plasma waves by a resonant streaming instability, similar to the creation (Kulsrud 2005) of ion Alfvén waves by streaming relativistic cosmic ray nuclei. But these do not appear to be effective either. These $\sim \mathrm{MeV}$ positrons generate resonant whistler waves (Schlickeiser 2002, page 339) at wavelengths significantly less than the scale of the thermal proton gyroradius, $r_{p}=u_{p} / \Omega_{p}$, at frequencies approaching those of thermal electrons, $\Omega_{e}$, where $u_{p}$ is the thermal proton speed. The phase speed of whistler waves in the direction of the mean field is the electron Alfvén speed, $V_{a e}=\left(m_{p} / m_{e}\right)^{1 / 2} V_{a}$.
If the positron streaming velocity, $V_{s}>V_{a e}$, and if the whistler waves were not damped so they grew to saturation, then the resulting magnetic field fluctuations in these waves would interact with the streaming positrons via quasi-linear waveparticle interactions that change their pitch angles, reducing $V_{s}$ to a level just less than $V_{a e}$. However, in predominantly neutral interstellar phases such whistler waves are also subject to severe ion-neutral damping (C. F. Kennel 2008, private communication).

Thus, we expect that positron streaming velocities along the magnetic flux tubes are comparable to their particle velocities, $\bar{\beta} c$, similar to the very high drift speeds found (Felice \& Kulsrud 2001) for cosmic ray nuclei in warm H I regions. Nonetheless, in turbulent media the flux tubes themselves may essentially random walk, so that the propagation is described by so-called "compound diffusion" (Lingenfelter et al. 1971).

\section{POSITRON PROPAGATION AND ANNIHILATION IN THE POSITRON BULGE}

Using these models of relativistic electron transport, we now investigate the propagation, slowing down, and annihilation of positrons produced in each of the different phases of the interstellar medium in the positron bulge, Galactic disk, and halo. The schematic model of the bulge, disk and halo is shown in Figure 1. Because the positrons, produced by SNIa and SNIp, are widely distributed in the bulge and disk (Equation (3)), we assume that the fraction produced in the various phases of the interstellar medium are proportional to their appropriate filling factors. Although the SNe themselves disturb the local medium, the $\sim \mathrm{MeV}$ positrons from the decay of radionuclei escape from the remnant into the undisturbed surroundings.

We estimate the relative probabilities, $P$, of positron annihilation within each phase and positron escape into neighboring phases by the following procedure. From the properties of the medium in each phase, we determine the propagation mode, diffusion or streaming, and calculate the diffusion mean free path $\lambda_{\|}$(Equation (6)) in the MHD scattering mode, or the mean velocity, $\bar{\beta} c$, in the unscattered streaming mode.

Although we have also made Monte Carlo simulations, we estimate the propagation of positrons during their slowing down in each phase of the interstellar medium, using a simple approximation. Since even the average properties and structure of the magnetic field especially are very poorly known in the various phases, no more sophisticated treatment seems justified. For the one-dimensional diffusion in a uniform medium with 
Table 1

Assumed Properties of the Galactic Interstellar Bulge $(<3.5 \mathrm{kpc})$

\begin{tabular}{|c|c|c|c|c|c|c|c|c|}
\hline \multirow{2}{*}{$\begin{array}{l}\text { Galactic } R \\
\text { Phase }\end{array}$} & \multicolumn{4}{|c|}{$\begin{array}{c}\text { Inner Bulge } \\
<0.5 \mathrm{kpc}\end{array}$} & \multicolumn{3}{|c|}{$\begin{array}{l}\text { Middle Bulge } \\
0.5-1.5 \mathrm{kpc}\end{array}$} & \multirow{2}{*}{$\begin{array}{c}\text { Outer Bulge } \\
1.5-3.5 \mathrm{kpc} \\
\text { Bo }\end{array}$} \\
\hline & $\mathrm{VH}$ & H II & H I & $\mathrm{H}_{2}$ & $\mathrm{HM}$ & H I & $\mathrm{H}_{2}$ & \\
\hline Filling Factor $f$ & 1 & 0.0002 & 0.0001 & 0.0004 & 1 & 0.00004 & 0.00005 & 1 \\
\hline Temperature $T(\mathrm{~K})$ & $(90-6) \times 10^{6}$ & 5000 & 150 & 30 & $5 \times 10^{6}$ & 20 & 20 & $1 \times 10^{6}$ \\
\hline Density $n \mathrm{H} \mathrm{cm}^{-3}$ & 0.04-0.007 & 100 & 1000 & $1900 \mathrm{H}_{2}$ & 0.005 & 1000 & $1000 \mathrm{H}_{2}$ & 0.002 \\
\hline Ionization Fraction $x$ & 1 & 1 & $3 \times 10^{-4}$ & $10^{-5}$ & 1 & $3 \times 10^{-4}$ & $10^{-5}$ & 1 \\
\hline Slowing Down $t_{\mathrm{sd}}(\mathrm{yr})$ & $(1-6) \times 10^{6}$ & 450 & 90 & 20 & $9 \times 10^{6}$ & 90 & 150 & $2 \times 10^{7}$ \\
\hline Magnetic Field $B \mu \mathrm{G}$ & $\sim 100-17$ & $\sim 100$ & $\sim 100$ & $\sim 100$ & $\sim 12$ & $\sim 12$ & $\sim 12$ & 4 \\
\hline Fluctuation $\delta B / B$ & $\sim 0.12-0.06$ & 1.3 & $\ldots$ & $\ldots$ & $\sim 0.03$ & $\ldots$ & $\ldots$ & $\sim 0.5$ \\
\hline Flux Tube $l_{B}(\mathrm{pc})$ & $10-300$ & $\sim 7$ & $\sim 5$ & $>7$ & $\sim 1000$ & $\sim 12$ & $\sim 5$ & $1000 \& 2000$ \\
\hline Max MHD Scale $l_{o}(\mathrm{pc})$ & $10-50$ & $\cdots$ & $\cdots$ & $\cdots$ & 50 & $\cdots$ & $\cdots$ & 75 \\
\hline$\lambda_{\|}(\mathrm{pc})$ & $25-30$ & 0.0017 & $\cdots$ & $\cdots$ & 45 & $\cdots$ & $\cdots$ & 14 \\
\hline Slowing Down $l_{\mathrm{sd}} \mathrm{pc}$ & $2000-5000$ & 0.3 & 12 & 3 & 7000 & 12 & 6 & 7000 \\
\hline$l_{B} /\left(l_{B}+l_{\mathrm{sd}}\right)$ & $0.01-0.03$ & 0.96 & 0.30 & $>0.70$ & 0.07 & 0.50 & 0.95 & $0.13 \& 0.22$ \\
\hline$Q_{X} 10^{43} e^{+} / \mathrm{s}$ & $0.31 \pm 0.07$ & $\sim 0$ & $\sim 0$ & $\sim 0$ & $0.37 \pm 0.09$ & $\sim 0$ & $\sim 0$ & $0.56 \pm 0.14$ \\
\hline
\end{tabular}

a mean free path $\lambda_{\|}$along a magnetic flux tube, $N$ positrons produced at a point, $l=0$ and $t=0$, will be distributed in both directions along the flux tube with a density, $n(l)=$ $\left(N / l_{\mathrm{sd}}\right) e^{-\left(l / l_{\mathrm{sd}}\right)^{2}}$, where $l_{\mathrm{sd}}=\left(2 \lambda_{\|} \beta c t_{\mathrm{sd}} / 3\right)^{1 / 2}$, by the time $t_{\mathrm{sd}}$ that they slow down and annihilate. This corresponds to a mean positron density $\bar{n}=N / 2 l_{\text {sd }}$ over the total mean length $2 l_{\mathrm{sd}}$. Similarly for positrons streaming at their velocity $\bar{\beta} c$ with an isotropic pitch angle distribution they will also have a mean density $N / 2 l_{\text {sd }}$ over a total mean length $2 l_{\mathrm{sd}}$, where $l_{\mathrm{sd}} \sim \bar{\beta} c t_{\mathrm{sd}} / 2$, when they slow down and annihilate.

Thus, if the positrons are produced uniformly along some mean length ${ }_{x} l_{B}$ of the magnetic flux tubes threading through some phase $x$, from the above we expect that the probability, $P_{x: x}$, that such positrons will slow down and annihilate in that phase before they escape is crudely,

$$
\begin{aligned}
& P_{x: x} \sim{ }_{x} l_{B} / 2_{x} l_{\mathrm{sd}} \text { for }{ }_{x} l_{B}<{ }_{x} l_{\mathrm{sd}} \text { and } \\
& P_{x: x} \sim{ }_{x} l_{B} /\left({ }_{x} l_{B}+{ }_{x} l_{\mathrm{sd}}\right) \text { for }{ }_{x} l_{B}>{ }_{x} l_{\mathrm{sd}},
\end{aligned}
$$

where ${ }_{x} l_{\mathrm{sd}}$ is the slowing down length in that phase. The remaining positrons have a probability, $1-P_{x: x}$, of escaping from phase $x$ into a neighboring phase, and we estimate the relative fractions of the escaping positrons that go into each of the adjacent phases from simple geometric arguments, allowing large uncertainties of $\pm 50 \%$.

The probability that positrons which diffuse into a phase $x$ will slow down and annihilate there is only slightly different from those born uniformly within it. These positrons are effectively born at their first scatter within the phase, roughly within a scattering mean free path of the boundary. So if ${ }_{x} l_{B}<_{x} l_{\mathrm{sd}}$, the probability of their slowing down and annihilating in the phase, $P_{x: x}$ is the same as for those born uniformly within, $\sim_{x} l_{B} / 2_{x} l_{\mathrm{sd}}$. But if ${ }_{x} l_{B}>_{x} l_{\text {sd }}$, then $P_{x: x}$ remains at $\sim 1 / 2$, since after the first scatter near the boundary half would be expected to be spread over ${ }_{x} l_{\mathrm{sd}}$ in both directions along the flux tube by the time they slow down and annihilate. Thus only about half of them will slow down and annihilate in phase $x$, while the other half will escape, or be effectively reflected. As we show, however, in the dense labyrinth of clouds in the CMZ and tilted disk the half of the positrons that diffuse into clouds from the VH and $\mathrm{HM}$ and are reflected back out rather than annihilating within, quickly diffuse into another cloud where half are again reflected, and by the time they have encountered a half dozen other clouds only a small fraction, $\sim(1 / 2)^{7}$, or $<1 \%$ remain. As we also show, however, in the diffuse disk beyond $1.5 \mathrm{kpc}$ that is not the case.
From these simple approximations, we estimate the different propagation fractions $P$ that relate the positron production rates $Q$ and annihilation rates $A$, as defined in Equation (4) for the expected bulge/disk annihilation ratio, expanding that equation to explicitly define these fractions in the different phases of the interstellar medium within the bulge, disk, and halo.

We consider here the Galactic positron bulge as it is defined by the spherical component $(<1.5 \mathrm{kpc})$ of the $511 \mathrm{keV}$ luminosity in the SPI/INTEGRAL analyses (Knödlseder et al. 2005; Weidenspointner et al. 2007, 2008a). Also based on their model fitting we discuss it in two parts: the inner bulge region $(R<0.5 \mathrm{kpc})$ and the surrounding outer, or tilted-disk, bulge region $(0.5 \mathrm{kpc}<R<1.5 \mathrm{kpc})$.

In order to compare with the SPI/INTEGRAL measurements, we need to separate the positron production and annihilation in the bulge and disk into five regions, shown in Figure 1 , defined primarily by their galactocentic radius, although they also have very different mixes of interstellar phases, as recently reviewed by Ferrière et al. (2007). These are (1) the very hot, dense inner or nuclear bulge, $B i, \quad(<0.5$ $\mathrm{kpc})$, (2) the hot, middle bulge and tilted disk, $B m,(0.5-$ $1.5 \mathrm{kpc}$ ), (3) the hot, tenuous outer bulge, $B o$ between $\sim 1.5$ $\mathrm{kpc}$ and the Galactic molecular ring at around $\sim 3.5 \mathrm{kpc}$, dominated by the bulge wind (e.g., Bregman 1980; Blitz et al. 1993), (4) the predominantly warm neutral outer disk beyond $3.5 \mathrm{kpc}, D o$, and (5) the hot, tenuous halo, $H$, beyond $3.5 \mathrm{kpc}$ and above the disk. The properties of each of these regions, which we discuss below, are summarized in Tables 1 and 2 .

Based on the distributions of the surface density of the three positron source components, given in Equation (3): the bulge and disk distributions of SNIa and SNIp occurrences, and the disk distribution of massive (OB) stars (McKee \& Williams 1997), we estimated the fraction of positron production in each of these regions. The cumulative production as a function of Galactic radius is shown in Figure 2 for each component. We find that $45 \%, 38 \%$, and $17 \%$ of the positrons from the stellar bulge $B^{*}$ component of SNIa and SNIp are produced in the regions $<0.5 \mathrm{kpc}, 0.5$ to $1.5 \mathrm{kpc}$, and 1.5 to $3.5 \mathrm{kpc}$, respectively, plus $10 \%$ of the massive $s t a r{ }^{26} \mathrm{Al}$ component within $0.5 \mathrm{kpc}$. Of the positrons from the stellar disk $D *$ component of SNIa and SNIp, 2\%, 11\%, and 30\% are produced in those same regions, and the remaining 57\% are produced beyond $3.5 \mathrm{kpc}$, together with $90 \%$ of the massive $\operatorname{star}{ }^{26} \mathrm{Al}$ component. 
Table 2

Positron Propagation and Annihilation in the Galactic Interstellar Bulge $(<3.5 \mathrm{kpc})$

\begin{tabular}{lccccc}
\hline \hline Galactic $R$ & \multicolumn{2}{c}{$\begin{array}{c}\text { Inner Bulge } \\
<0.5 \mathrm{kpc}\end{array}$} & \multicolumn{2}{c}{$\begin{array}{c}\text { Middle Bulge } \\
0.5-1.5 \mathrm{kpc}\end{array}$} & $\begin{array}{c}\text { Outer Bulge } \\
1.5-3.5 \mathrm{kpc}\end{array}$ \\
\hline Phase $X$ & $\mathrm{VH}$ & H II & HM & H I & Bo \\
Filling Factor $f$ & 1 & $\sim 0$ & 1 & $\sim 0$ & 1 \\
$Q_{X} 10^{43} e^{+} / \mathrm{s}$ & $0.31 \pm 0.07$ & $\sim 0$ & $0.37 \pm 0.09$ & $\sim 0$ & $0.56 \pm 0.14$ \\
\hline
\end{tabular}

Propagation

Inner Bulge

$P_{\mathrm{VH}: \mathrm{X}}$
Middle Bulg

$P_{\mathrm{HM}: \mathrm{X}}$

Outer Bulge

$P_{B o: X}$

$\sim 0.96$

.

$\cdots$

$[\sim 0.02]$

.02

$\sim 0.22 \quad \sim \mathbf{0 . 0 7}$

$\sim 0.69$

$[\sim 0.02]$

Annihilation

Inner Bulge

$Q P_{\mathrm{VH}: \mathrm{X}} 10^{43} e^{+}$

Middle Bulge

$Q P_{\text {HM:X }}$

Outer Bulge

$Q P_{B e: X}$

$\sim 0.40$

$[\sim 0.14]$

$A_{X} 10^{43} e^{+} / \mathrm{s}$

$\sim 0.006$

$0.30 \pm 0.06$

$\cdots$

$[\sim 0.006]$

$0.08 \pm 0.04$

$\mathbf{0 . 0 3} \pm \mathbf{0 . 0 1}$

$0.26 \pm 0.13$

$[\sim 0.007]$

Thus the total positron production in the inner bulge $B i$ within $0.5 \mathrm{kpc}$, the middle bulge $B m$ between 0.5 and $1.5 \mathrm{kpc}$ and the outer bulge $B o$ between 1.5 and $3.5 \mathrm{kpc}$, including both stellar bulge and disk components, and the production in the outer disk Do beyond $3.5 \mathrm{kpc}$, are

$$
\begin{aligned}
Q_{B i} & =\left[(4.1 \pm 1.6) f_{56}+(0.10 \pm 0.05)\right] \times 10^{43} \mathrm{e}^{+} \mathrm{s}^{-1} \\
Q_{B m} & =\left[(5.7 \pm 2.3) f_{56}+(0.09 \pm 0.04)\right] \times 10^{43} \mathrm{e}^{+} \mathrm{s}^{-1}, \\
Q_{B o} & =\left[(8.6 \pm 3.5) f_{56}+(0.13 \pm 0.05)\right] \times 10^{43} \mathrm{e}^{+} \mathrm{s}^{-1}, \\
Q_{D o} & =\left[(13.7 \pm 5.6) f_{56}+(0.48 \pm 0.24)\right] \times 10^{43} \mathrm{e}^{+} \mathrm{s}^{-1}
\end{aligned}
$$

And using the best-fit positron survival fraction, $f_{56} \sim 5 \% \pm$ $2 \%$ from Section 2.2 above, the total positron production rates are

$$
\begin{aligned}
Q_{B i} & =(0.31 \pm 0.07)] \times 10^{43} \mathrm{e}^{+} \mathrm{s}^{-1}, \\
Q_{B m} & =(0.37 \pm 0.09)] \times 10^{43} \mathrm{e}^{+} \mathrm{s}^{-1}, \\
Q_{B o} & =(0.56 \pm 0.14)] \times 10^{43} \mathrm{e}^{+} \mathrm{s}^{-1}, \\
Q_{D o} & =(1.16 \pm 0.20)] \times 10^{43} \mathrm{e}^{+} \mathrm{s}^{-1} .
\end{aligned}
$$

Within each of these regions, $y$, we determine the expected positron production rates in each separate interstellar gas and plasma phase, $x$, by the relative filling factors of those phases, $f_{y x}$, times the production rate in that region, $Q_{y}$, such that $Q_{y x} \sim f_{y x} Q_{y}$. We then estimate the final positron annihilation rates, $A_{y^{\prime} x^{\prime}}$, in each phase $x^{\prime}$ within each region $y^{\prime}$, as the sums over $x, y, x^{\prime}$ and $y^{\prime}$ of products of the production rates, $Q_{y x}$, times the propagation fractions, $P_{y x: y^{\prime} x^{\prime}}$, where each is the fraction of the positrons born in $y x$ that propagate to and annihilate in $y^{\prime} x^{\prime}$, as discussed above and given for $x: x$ in Equation (8).

In the next three sections, we estimate these propagation fractions from modeling the propagation, slowing down and annihilation of positrons in each of these regions and phases starting with the Galactic bulge and moving outward in the disk and ultimately into the halo, which is an important region of propagation and annihilation, despite its lack of local production.

$$
\text { 4.1. Inner Bulge }(R<0.5 \mathrm{kpc})
$$

We model the complex interstellar phenomena of the inner region of the Galaxy $(\mathrm{R}<0.5 \mathrm{kpc})$, assuming that a very hot

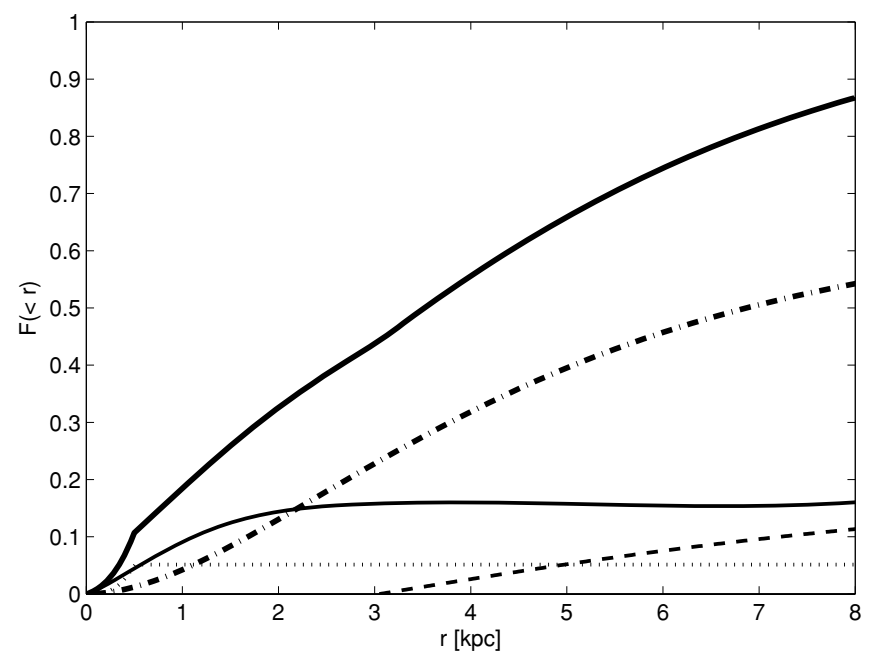

Figure 2. Cumulative fraction of total (thick solid curve) Galactic positron production $F(<r)$ in the bulge and disk as a function of Galactic radius $r$ from Equations (3) and (4). The assumed distribution of positrons from decay of ${ }^{56} \mathrm{Ni}$ and ${ }^{44} \mathrm{Ti}$, produced by SNIa and SNIp, are shown for the stellar disk (dot-dashed curve), bulge (thin solid curve) and a star burst near the Galactic center (dotted curve), while those from decay of ${ }^{26} \mathrm{Al}$, produced by massive WR stars and core-collapse $\mathrm{SNe}$ are shown in the short dashed curve.

$\left(\sim 10^{8} \mathrm{~K}\right)$, high-pressure plasma permeates the region, pressure equilibrium exists among the interstellar phases (Spergel \& Blitz 1992; Carral et al. 1994), and the properties of the HII, H I, and $\mathrm{H}_{2}$ regions are related to each other via the scenario of photodissociation regions (PDRs; Tielens \& Hollenbach 1985).

$\mathrm{O}$-star radiation is the primary photoionization source in the interstellar medium (McKee \& Williams 1997). Outside of galactic centers, ultraviolet radiation flux at an arbitrary location in galactic nuclear regions is generated by nearby $\mathrm{OB}$ associations, or by single $\mathrm{O}$ stars distributed randomly throughout the regions (Wolfire et al. 1990). However, in the immediate vicinity of a galactic center ionizing radiation is dominated by the radiation contribution of active galactic nuclei. In the inner Galactic bulge the bulk of the ionizing radiation seems to be generated by randomly distributed 


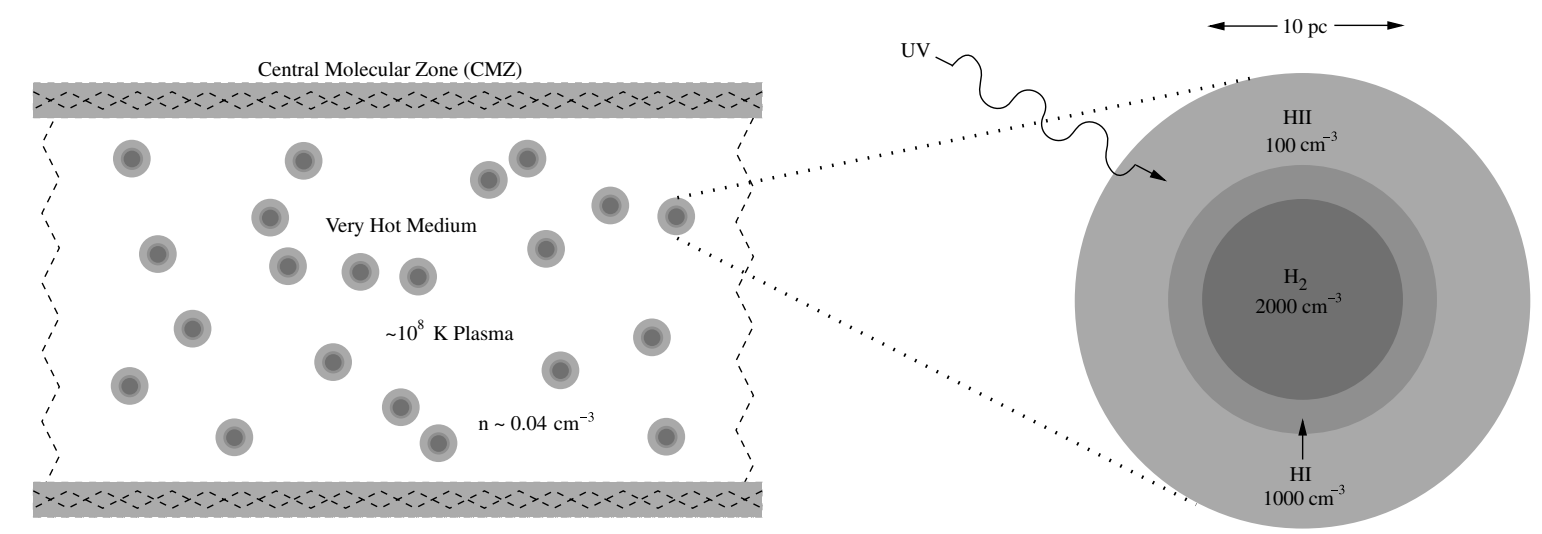

Figure 3. Schematic view of molecular clouds in the $\mathrm{CMZ}$ of the inner bulge within $0.5 \mathrm{kpc}$ of the Galactic center, together with a cross section of the $\mathrm{H}$ II and $\mathrm{H}$ I envelopes surrounding the $\mathrm{H}^{2}$ core of a model cloud.

O-stars which photoionize the outer layers of nearby interstellar clouds (Wolfire et al. 1990; Carral et al. 1994). However, in the outer $(>3.5 \mathrm{kpc})$ Galaxy the great majority of $\mathrm{O}$ stars are clumped in OB associations, which in turn create dense, compact $\mathrm{H}$ II regions, which surround such OB associations (McKee \& Williams 1997). In the outer galaxy, only, small fraction $(<15 \%)$ of ionizing radiation generated in these $\mathrm{OB}$ associations escapes to maintain the diffuse $\mathrm{H}$ II.

Thus the photoionized, as well as the neutral, phases in the centers of galaxies have been modeled as ensembles of PDRs (Wolfire et al. 1990; Carral et al. 1994). Each cloud is viewed as having a spherical molecular core of radius, $r_{\mathrm{H}_{2}}$, which contains the bulk of the cloud mass at a molecular hydrogen density $n_{\mathrm{H}_{2}}$. Surrounding the molecular cores are cold atomic H I envelopes, $\Delta r_{\mathrm{H}_{\mathrm{I}}}$ thick, which in turn generate infrared continua and fine-structure line emissions (Wolfire et al. 1990). Finally, envelopes of photoionized $\mathrm{H}$ II plasma form outer shells, $\Delta r_{\mathrm{H} \text { II }}$ thick, surrounding these H I shells (Carral et al. 1994), ionized by Lyman continuum emission from randomly distributed hot, massive stars within the central $0.5 \mathrm{kpc}$. Following Caral et al., we assume that these $\mathrm{H}$ II envelopes constitute the primary photoionized gas component and a schematic model of the clouds is shown in Figure 3. Although the hot, massive $\mathrm{O}$ stars that generate the $\mathrm{H}$ II ionizing emission are thought to occur only in the CMZ, we expect that clouds in the inner portion of the surrounding tilted disk are also likely to be irradiated with sufficient flux to maintain H II envelopes. The extent of such irradiation is not known, however, and here we arbitrarily assume that it extends only to $0.5 \mathrm{kpc}$.

We also assume that the magnetic flux tubes nominally pass through the cold clouds in a parallel array, but with large turbulent perturbations superimposed, as suggested by magnetic field in giant molecular clouds. Li et al. (2006), using the SPARO $450 \mu \mathrm{m}$ polarimetry observations, have found that within some such clouds in the disk, the direction of the magnetic field is roughly correlated with that of the local Galactic field. However, extensive Zeeman measurements of molecular clouds also indicate strong field perturbations from supersonic motions driven by MHD turbulence with energies comparable to that of the magnetic fields within the clouds (see reviews by Crutcher 1999; Falceta-Goncalves et al. 2008).

Thus, we assume a nominal field to calculate the base mean length of flux tubes through the clouds, and then from an estimate of the scale length of turbulent motions, we estimate the increase of that mean length resulting from the effective random walk or meandering of the flux tubes through the turbulent perturbations. The nominal mean flux tube length through cold cloud cores is $l_{B}^{\prime} \sim 4 r / 3$, where $r$ is the core radius, while the nominal mean length through an overlying shell of thickness $\Delta r$, is $l_{B}^{\prime} \sim(4 / 3)\left[(r+\Delta r)^{3}-r^{3}\right] /(r+\Delta r)^{2}$. Conservatively taking the outer scale of turbulent motion $l_{o}$ as the step size, which minimizes the random walk, we expect a mean meandering flux tube length, $l_{B} \sim\left(l_{B}^{\prime} / l_{o}\right) l_{B}^{\prime}$.

In the inner bulge the molecular clouds concentrate into two nested disks, the CMZ and the surrounding tilted disk, which extends well beyond $0.5 \mathrm{kpc}$ out to edge of the middle bulge at $1.5 \mathrm{kpc}$. The CMZ is a highly asymmetric region, which is an ellipsoidal disk, $\sim 40 \mathrm{pc}$ thick vertically, with lateral axes of $\sim 500$ pc by $\sim 250$ pc and an interstellar $\mathrm{H}_{2}$ mass of $1.9 \times 10^{7} M_{\odot}($ Ferrière et al. 2007). In this region we estimate a mean molecular cloud core mass, $M_{\mathrm{H}_{2} \text { core }} \sim 5 \times 10^{4} M_{\odot}$ and a mean core radius, $r_{\mathrm{H}_{2}} \sim 5 \mathrm{pc}$, from integrations of the mass spectrum $\left(2 \times 10^{3} \mathrm{M}_{\odot} \leqslant \mathrm{M}_{\mathrm{H}_{2} \text { core }} \leqslant 2 \times 10^{6} M_{\odot}\right)$ and the size spectrum (3.3 pc (resolution limit) $\leqslant r_{\mathrm{H}_{2}} \leqslant 10^{2} \mathrm{pc}$ ) of the CMZ molecular cloud population determined by Miyazaki \& Tsuboi (2000). This implies a total of $\sim 400$ such cloud cores with a $\mathrm{H}_{2}$ density $n_{\mathrm{H}_{2}} \sim 1900 \mathrm{~cm}^{-3}$. It also gives a total volume filling factor of these $\mathrm{H}_{2}$ clouds in the $\mathrm{CMZ}$ is $f_{\mathrm{H}_{2}} \sim 0.08$, but in the full inner bulge $<0.5 \mathrm{kpc}$ volume, the filling factor is only $f_{\mathrm{H}_{2}} \sim 4 \times 10^{-4}$.

The much larger tilted disk, which is described in more detail in the next subsection (4.2), surrounds the CMZ, extending out to $\sim 1.5 \mathrm{kpc}$ with an estimated interstellar $\mathrm{H}_{2}$ mass of $3.4 \times$ $10^{7} M_{\odot}$ (Ferrière et al. 2007). About $20 \%$ of the tilted disk lies within the bulge source region $<0.5 \mathrm{kpc}$. This inner portion of the Titled Disk contains $\sim 7 \times 10^{6} M_{\odot}$ of interstellar $\mathrm{H}_{2}$. We assume that this $\mathrm{H}_{2}$ gas is also concentrated in similar molecular cloud cores, which would number $\sim 140$, if they have the same mean density and mass as those in the CMZ, and they would have an even smaller filling factor in the bulge source volume $<0.5 \mathrm{kpc}$.

Within and surrounding the CMZ, containing the molecular clouds, is a very hot tenuous plasma (VH). Observations of 6.7 $\mathrm{keV}$ line emission from the $\mathrm{K} \alpha \mathrm{He}$-like transition of ionized $\mathrm{Fe}$ indicate that this $\mathrm{VH}$ plasma has a temperature of $\sim 10^{8} \mathrm{~K}$, and fills most of this central region (Koyama 1989; Yamauchi et al. 1990; Spergel \& Blitz 1992; Koyama et al. 1996; Muno et al. 2004). Thus we assume that a hot $\left(\sim 9 \times 10^{7} \mathrm{~K}\right)$, plasma $(n \sim$ $0.04 \mathrm{~cm}^{-3}$ ) fills the inner $\sim 500 \mathrm{pc}$ of the bulge (e.g., Yamauchi et al. 1990). The thermal pressure, $p_{t h} / k=n_{\mathrm{VH}} T_{\mathrm{VH}} / \mu \sim 10^{7}$ $\mathrm{K} \mathrm{cm}^{-3}$, where $\mu$ represents the mean particle mass for fully ionized $\mathrm{H}$, and $k$ is Boltzmann's constant. This value agrees well with previous estimates of the CMZ thermal pressure of $\sim 5 \times 10^{6} \mathrm{~K} \mathrm{~cm}^{-3}$ by Spergel \& Blitz (1992). 
Analyzing the diffuse nonthermal radio emission of the Galactic center region, Spergel \& Blitz (1992) found that the magnetic field pressure is in approximate equilibrium with the thermal gas pressure. Consequently, we assume that both the intercloud and cloud field strengths are $\sim 100 \mu \mathrm{G}$, the value expected from such pressure arguments, $5 \times 10^{-10} \mathrm{erg} \mathrm{cm}^{-3}$.

Between the VH plasma and the $\mathrm{H}_{2}$ core of each cloud are the two surrounding envelopes, the upper warm, ionized $\mathrm{H}$ II gas and the lower, cold neutral $\mathrm{H}$ I gas. The H II density is estimated from pressure balance between $\mathrm{HII}$ gas and the VH (Carral et al. 1994). Although the thermal pressure of the overlying $\mathrm{VH}$ is approximately matched by the total pressure of the cold medium $\left(\mathrm{H}_{2}\right)$, the latter pressure is dominated by turbulence (Tielens \& Hollenbach 1985; Spergel \& Blitz 1992; Oka et al. 1998). Similarly, the large line widths observed in the extended H II gases in the Galactic center region (e.g., RodriguezFernandez \& Martin-Pintado 2005) imply that turbulence also dominates the $\mathrm{H}$ II pressure. We assume that the magnetic field strengths are the same in the VH plasma and the $\mathrm{H}$ II gas. Thus we find that a turbulent $\mathrm{H}_{\text {II }}$ pressure, $p_{t u r b}=\frac{1}{2} \rho_{\mathrm{H} \text { II }} \overline{\left(u_{\mathrm{HII}}^{2}\right)}$ (Tielens \& Hollenbach 1985), constituting $\sim 85 \%$ of the total H II pressure equal to the total $\mathrm{VH}$ pressure, possesses an rms turbulent velocity, $\left({\overline{\left(\mathrm{H}_{\mathrm{HI}}^{2}\right.}}^{1 / 2}\right.$, of $\geqslant 25 \mathrm{~km} \mathrm{~s}^{-1}$, for a mass density, $\rho_{\mathrm{H} \text { II }}$, corresponding to $n_{\mathrm{H} \text { II }} \sim 100 \mathrm{~cm}^{-3}$. Note that the $\mathrm{H}$ II thermal pressure, $2 n_{\mathrm{H} \text { II }} T_{\mathrm{H} \text { II }}, \sim 10^{6} \mathrm{~K} \mathrm{~cm}^{-3}$, is small compared to that of the $\mathrm{VH}$, since $T_{\mathrm{H} \text { II }}$ is $\sim 5000 \mathrm{~K}$ (Mezger \& Pauls 1979).

Based on Wolfire et al. (2003), we expect that at the high thermal pressures found in the Galactic nuclear region, warm $\mathrm{H} \mathrm{I}$ is unstable and only cold $\mathrm{H}_{\mathrm{I}}$ is thermally stable. From analyses of fine structure emissions from PDRs of CMZ molecular clouds located far from thermal radio sources Rodríguez-Fernández et al. (2004) found typical H I densities, $n_{\mathrm{HI}}$, of $\sim 1000 \mathrm{~cm}^{-3}$ with $T_{\mathrm{HI}} \sim 150 \mathrm{~K}$. For $R<0.5 \mathrm{kpc}$, Ferrière et al. estimated an interstellar $\mathrm{H}$ I mass of $1.7 \times 10^{6} M_{\odot}$, with a space averaged density, $\left\langle n_{\mathrm{HI}_{\mathrm{I}}}\right\rangle \sim 25 \mathrm{~cm}^{-3}$ in the $\mathrm{CMZ}$ and a filling factor there of $f_{\mathrm{HI}} \sim 0.025$. At such a mean density, the typical thickness of cold atomic shells is expected to be $\Delta r_{\mathrm{HI}} \sim 0.5 \mathrm{pc}$. The typical thickness of $\mathrm{H}$ II shells, surrounding such $\mathrm{H}$ I shells with a filling factor $f_{\mathrm{H} \text { II }} \sim 0.045$, is expected to be $\Delta r_{\mathrm{H}} \sim 0.7 \mathrm{pc}$. Thus, the outer radius of a typical, double-shelled CMZ cloud, $r_{c} \sim 6.2 \mathrm{pc}$. The total cloud filling factor in the CMZ, $f_{c}=f_{\mathrm{H}_{2}}+f_{\mathrm{H} \text { II }}+f_{\mathrm{HI}}$ is $\sim 0.15$ and it further implies a mean distance between cloud centers $d_{c c} \sim 2 r_{c} f_{c}^{-1 / 3}$ of only $\sim 23 \mathrm{pc}$ and that between cloud surfaces, $d_{c c}-2 r_{c}$ of just $\sim 11 \mathrm{pc}$.

As we will show, the annihilation of the positrons takes place almost entirely in the cloud shells. However, only a negligible fraction $(<1 \%)$ of them are produced there, since the filling factors of the cloud phases are all negligible compared to the inner bulge volume. Thus we assume that all of the inner bulge positrons are born in the pervasive, very hot medium, $Q_{B i V H} \sim Q_{B i} \sim(0.31 \pm 0.07) \times 10^{43} \mathrm{e}^{+} \mathrm{s}^{-1}, Q_{B i H I I} \sim$ $Q_{B i H I} \sim Q_{B_{B i}} \sim 0$.

The subsequent positron annihilation in these phases of the bulge is then defined by the propagation fractions, which we estimate below using this general model of the CMZ. Because, as we show, the positron slowing down and annihilation in the $\mathrm{H}$ II phase of CMZ is so efficient, the probability of positrons, formed in the $\mathrm{VH}$, penetrating through the $\mathrm{H}$ II into the underlying $\mathrm{HI}_{\mathrm{I}}$ and $\mathrm{H}_{2}$ is negligible, so we only show here the terms for diffusion between the other adjacent phases with significant filling factors. We also find that the probability of positrons successfully escaping out of the inner disk is quite small.

Thus the significant terms in each phase are

$$
\begin{array}{r}
A_{B i V H} \sim P_{\mathrm{VH}: \mathrm{VH}}\left(Q_{B i V H}+Q_{B m} P_{B m: B i V H}\right. \\
\left.+Q_{B o} P_{B o: B i V H}\right), \\
A_{B i H I I} \sim P_{\mathrm{H} \text { II:H II }}\left(Q_{B i H I I}+Q_{B i V H} P_{\mathrm{VH}: \mathrm{H} \mathrm{II}}\right. \\
\left.+Q_{B m} P_{B m: B i H I I}+Q_{B o} P_{B o: B i H I I}\right), \\
A_{B i H I} \sim A_{B i H_{2}} \sim 0,
\end{array}
$$

where, like those discussed above, the propagation fractions $P$, subscripted $\mathrm{VH}: \mathrm{VH}$ is the fraction of the positrons produced in the $\mathrm{VH}$ phase that slow down and annihilate in that phase, $\mathrm{VH}: \mathrm{H}$ II is the fraction that escaped from it into the adjacent $\mathrm{H}$ II, and other fractions are similar. The propagation fractions and the resultant annihilation rates are listed in Table 2.

\subsubsection{Very Hot Medium (VH)}

The bulk of the positrons from the inner bulge SNIa and SNIp are expected to be born in the $\mathrm{VH}$, which includes both the high density and temperature plasma in the CMZ and all of the lower density and temperature hot plasma that fills nearly $\sim 100 \%$ of the inner bulge $<0.5 \mathrm{kpc}$. To determine the propagation fractions within the $\mathrm{VH}$, and between it and the $\mathrm{H}$ II regions, we estimate the median distance from the positron production site to the $\mathrm{H}$ II envelopes around clouds in the CMZ and inner tilted disk. We consider separately the two distributed source components: the compact $57 \%$ of the inner bulge SNIa and SNIp, resulting from the star burst and inner disk population, and the diffuse $43 \%$, resulting from the $\mathrm{SNe}$ in the general bulge population that are expected to occur throughout the inner bulge.

For the star burst and disk component with positron production uniformly distributed sources in the CMZ with a mean distance between $\mathrm{H}$ II shells would be $d \sim 10 \mathrm{pc}$, suggests that in a fairly regular magnetic field the mean flux tube length $l_{B}$ would also be $\sim 10 \mathrm{pc}$ in the CMZ. On the other hand, for the bulge component with the production roughly uniform throughout the spherical volume, the positrons would be distributed along a mean distance $d \sim(4 / 3) \pi r^{3} / 2 \pi r^{2} \sim(2 / 3) r \sim 300 \mathrm{pc}$ both above and below the CMZ with similar mean flux tube lengths, $l_{B}$, along the roughly vertical magnetic field in that region (e.g., Beck 2001).

In order to determine what fraction of the positrons, born in the VH, can slow down and annihilate there and what fraction escape into the adjacent $\mathrm{H}$ II envelopes, we also need to estimate the diffusion mean free path of these positrons in the VH plasma.

It is difficult to quantify the properties of hypothetical turbulent flows in the $\mathrm{VH}$, since the $\mathrm{VH}$ origin and age are unknown. However, a plausible constraint on such turbulence is that the dissipation rate of the turbulent energy must be less than the cooling rate of the VH plasma. The rate, at which turbulent energy is dissipated is $\sim \rho_{\mathrm{VH}}\left(\overline{u_{\mathrm{VH}}^{2}}\right)^{3 / 2} / l_{o}$, in units of $\mathrm{erg} \mathrm{cm}^{-3} \mathrm{~s}^{-1}$, where $\rho_{\mathrm{VH}}$ is the mass density of the $\mathrm{VH},\left(\overline{u_{\mathrm{VH}}^{2}}\right)^{1 / 2}$ is the rms turbulent velocity, and $l_{o}$ is the outer scale of turbulence (Townsend 1976).

From the X-ray observations Muno et al. (2004) estimated that the VH cooling time, $t_{\text {cool }} \sim 10^{8}$ yr. Since the VH thermal energy density, $\epsilon_{t h} \approx \frac{3}{2} p_{t h}$, the cooling constraint becomes $\left(\overline{u_{\mathrm{VH}}^{2}}\right)^{1 / 2} \sim\left(l_{o} \epsilon_{t h} /\left(t_{\text {cool }} \rho_{\mathrm{VH}}\right)\right)^{1 / 3}$. Taking the maximum estimate of $l_{o} \sim 10 \mathrm{pc}$, the mean separation between cloud surfaces, this 
relation limits $\left(\overline{u_{\mathrm{VH}}^{2}}\right)^{1 / 2}$ to less than $50 \mathrm{~km} \mathrm{~s}^{-1}$, which is much less than the VH Alfvén speed, $V_{a} \sim 920 \mathrm{~km} \mathrm{~s}^{-1}$. This cooling relation places a severe constraint on $\mathrm{VH}$ turbulence, if the $\mathrm{VH}$ is long lived on the timescale of $t_{\text {cool. }}$. However, if the VH is a transient phenomenon, this constraint could be weakened and the turbulence would be stronger.

Thus, if the mechanism generating the VH turbulence operated over a duration, $t_{m}$, less than $t_{\text {cool }}$, the dissipation rate of the turbulent energy can be greater, $\sim E_{t h} / t_{m}$. Thus $\left(\overline{u_{\mathrm{VH}}^{2}}\right)^{1 / 2} \sim$ $\left(l_{o} \epsilon_{t h} /\left(t_{m} \rho_{\mathrm{VH}}\right)\right)^{1 / 3}$. If, very conservatively, $t_{m} \sim 25 \mathrm{Myr}$, then $\left(\overline{u_{\mathrm{VH}}^{2}}\right)^{1 / 2}$ is less than $90 \mathrm{~km} \mathrm{~s}^{-1}$. Since in strong turbulent MHD flows, where energy is equipartitioned between magnetic and velocity fields $\left\langle\delta B_{\mathrm{VH}}^{2}\right\rangle \sim 4 \pi \rho_{\mathrm{VH}}\left\langle u_{\mathrm{VH}}^{2}\right\rangle$ s (e.g., Iroshnikov 1964; Kraichnan 1965; Biskamp 2003), constraining $\left\langle u_{\mathrm{VH}}^{2}\right\rangle$ limits $\left\langle\delta B_{\mathrm{VH}}^{2}\right\rangle$, and then $\left\langle\delta B_{\|}^{2}\right\rangle^{1 / 2}$, since $\left\langle\delta B_{\|}^{2}\right\rangle^{1 / 2} \sim 0.15\left\langle\delta B_{\mathrm{VH}}^{2}\right\rangle^{1 / 2}$. Thus $\left(B_{o} / \delta B_{\|}\right)^{2} \sim 800$, and, consequently a rather large $\lambda_{\|}$ $\sim 25 \mathrm{pc}$ in the CMZ from Equation (6).

In the VH the positron slowing down time is $t_{\mathrm{sd}} \sim 9 \times$ $10^{4} / n\left(1+x_{e}\right) \sim 1.1 \times 10^{6} \mathrm{yr}$ from Equation (5) at $n \sim 0.04$ $\mathrm{cm}^{-3}$. In the slowing down time, $t_{\mathrm{sd}}$, the positrons diffusing along a flux tube in the $\mathrm{VH}$ will be distributed over a mean length $l_{\mathrm{sd}} \sim\left(2 \lambda_{\|} \bar{\beta} c t_{\mathrm{sd}} / 3\right)^{1 / 2} \sim 2000 \mathrm{pc}$, since the average positron velocity during deceleration is $\sim 0.7 c$.

Roughly $61 \%$ of the inner bulge positrons are expected to occur in the CMZ, predominantly from the SNIa and SNIp in the star burst and the inner disk, along with $10 \%$ of the massive OB stars producing ${ }^{26} \mathrm{Al}$. The remaining $39 \%$, all from SNIa and SNIp, are expected to occur throughout the nuclear bulge $<0.5 \mathrm{kpc}$. This mean diffusion length is much larger than the mean flux tube length, $l_{B}$, for positrons escaping into the H II. For those produced by the star burst and disk component of SNIa and SNIp in the CMZ and inner disk, where the mean distance between the HII envelopes of neighboring clouds, $l_{B}$ is only $\sim 10 \mathrm{pc}$, we would expect from Equation (8) that $P_{\mathrm{VH}: \mathrm{VH}} \sim l_{B} / 2 l_{\mathrm{sd}}<0.01$, so only a negligible fraction would slow down and annihilate in the $\mathrm{VH}$, while nearly all would diffuse into the H II.

The much more extended positron production by the bulge $\mathrm{SNe}$ fills the inner bulge more uniformly within $0.5 \mathrm{kpc}$. Within the full volume the mean density of the $\mathrm{VH}$ is closer to $\sim 0.007 \mathrm{~cm}^{-3}$ and the temperature is much lower, $\sim 6 \times 10^{6} \mathrm{~K}$ (Almy et al. 2000). Assuming a mean $B_{o} \sim 17 \mu \mathrm{G}$ in equilibrium with the thermal pressure, and taking the same $t_{m}$ of $25 \mathrm{Myr}$, and a longer $l_{o} \sim 50 \mathrm{pc}$, we expect a mean free path $\lambda_{\|} \sim 30 \mathrm{pc}$ from Equation (6). Thus, with a slowing down time from Equation (5) of $t_{\mathrm{sd}} \sim 6 \times 10^{6} \mathrm{yr}$, the slowing down length $l_{\mathrm{sd}} \sim 5000 \mathrm{pc}$, while the median flux tube length of $l_{B} \sim 300 \mathrm{pc}$ between the CMZ and the middle bulge, so $P_{\mathrm{VH}: \mathrm{VH}} \sim 0.03$.

Thus, weighting these two probabilities with their relative (0.61:0.39 including $\left.{ }^{26} \mathrm{Al}\right)$ production fractions, we would expect the overall $P_{\mathrm{VH}: \mathrm{VH}} \sim 0.02$. Monte Carlo simulations give the same result. They also suggest that less than $2 \%$ of the positrons escape beyond the bulge $(>1.5 \mathrm{kpc})$, since they tend to be reflected, because the scattering mean free path there is much shorter and the slowing down time is much longer, so there is little likelihood of their slowing down and annihilating there before they diffuse back out and eventually diffuse into the HII below. Although, as we discussed above, about half of the positrons entering the turbulent $\mathrm{HII}$ envelopes are also reflected, because of the closeness of clouds, positrons escaping from one cloud envelope quickly enter another, so after just half a dozen cloud encounters nearly all have slowed down and annihilated in the $\mathrm{H}$ II. Therefore, we conclude that the effective propagation factors are $P_{\mathrm{VH}: \mathrm{VH}} \sim 0.02$ and $P_{\mathrm{VH}: \mathrm{H} \mathrm{II}} \sim 0.96$, while $P_{V H: B e} \sim 0.02$.

Thus, only $2 \%$ of the positrons born in the $\mathrm{VH}$ and diffusing along meandering magnetic flux tubes, would be expected to slow down and annihilate there before they moved into the $\mathrm{H}$ II shells surrounding the molecular clouds.

\subsubsection{Photoionized Medium (H II)}

From the source-weighted filling factors we expect that only $\sim 0.03$ of the SNIa and SNIp positrons are born in the warm ionized $\mathrm{H}$ II phase, but over an order of magnitude more positrons are expected to diffuse into it from the surrounding VH.

The highly turbulent nature of the H II medium follows from observations of the CMZ that reveal extreme radio-wave scattering by small-scale, turbulent density fluctuations (Lazio \& Cordes 1998; Goldreich \& Sridhar 2006). Although the origin of such intense small-scale turbulence is still uncertain, such small-scale fluctuations are expected to occur in the ionized interfaces between the dense, molecular cloud cores and the $\mathrm{VH}$ (Lazio \& Cordes 1998; Goldreich \& Sridhar 2006).

As discussed previously, the $\mathrm{H}$ II shells around molecular cloud cores have a density $n_{\mathrm{H} \text { II }} \sim 100 \mathrm{~cm}^{-3}$ and a thickness, $\Delta r_{\mathrm{H} \text { II }} \sim 0.7$ pc. The electron fraction high, $x_{e} \sim 1$, in view of the expected presence of the small-scale, ionized density fluctuations. Thus, a positron slowing down time, $t_{\mathrm{sd}}$, is $\sim 450 \mathrm{yr}$ from Equation (5). We model the spatial scale of the $\mathrm{H}$ II turbulence, $l_{o} \sim 0.25 \Delta r_{\mathrm{H} \text { II }} \sim 0.2 \mathrm{pc}$, assuming that the properties of such turbulence can be approximated by those generated by shear flows in channels $\Delta r_{\mathrm{H} \text { II }}$ wide (Pope 2000). Further, we find that $\left(\overline{u_{\mathrm{HII}}^{2}}\right)^{1 / 2} \sim 25 \mathrm{~km} \mathrm{~s}^{-1}$ from our assumption that turbulent pressure is about 6 times that of the thermal pressure. From Equation (6) we expect that $\lambda_{\|}$is very small, $\sim 1.7 \times 10^{-3} \mathrm{pc}$. In the slowing down time, $t_{\mathrm{sd}}$, these positrons diffusing along a flux tube in the $\mathrm{H}$ II plasma will be distributed over a mean length $l_{\mathrm{sd}} \sim\left(2 \lambda_{\|} \bar{\beta} c t_{\mathrm{sd}} / 3\right)^{1 / 2} \sim 0.3 \mathrm{pc}$.

This positron slowing down distance is less than the expected nominal mean length of a flux tube through the shells, $l_{B}^{\prime} \sim$ $(4 / 3)\left[(r+\Delta r)^{3}-r^{3}\right] /(r+\Delta r)^{2} \sim 1.2 \mathrm{pc}$, and much less than that expected for the meandering flux tubes in the turbulent ionized gas, which effectively random walk on the scale of the turbulence, $l_{o}$. For a turbulent scale equal to a quarter of the $\mathrm{H}$ II shell thickness or $\sim 0.2 \mathrm{pc}$, as discussed above, the mean meandering flux tube length would be $\left.l_{B} \sim\left(l_{B}^{\prime} / l_{o}\right) l_{B}^{\prime}\right) \sim 7.2 \mathrm{pc}$, or more than 20 times the slowing down length.

From the filling factor we expect only a negligible fraction of the positrons to be born in the H II, while, as we saw, essentially all $(\sim 96 \%)$ of the positrons born in the inner bulge, are expected to diffuse into the $\mathrm{H}$ II envelopes from the $\mathrm{VH}$. There in each encounter, as we discussed above, roughly $50 \%$ are expected to quickly slow down and annihilate within the outer $l_{\mathrm{sd}}$, which is much less than the mean flux tube length $l_{B}$ through the HII. Since these positrons are effectively born close to the boundary, the other half are expected to escape back into the VH. But there, because of the close proximity of the clouds, the escaping positrons quickly diffuse into another $\mathrm{H}$ II envelope, where the process is repeated, and soon virtually all are slowed down and annihilated in the $\mathrm{H}$ II. Thus the net propagation fractions are effectively $P_{\mathrm{H}_{\text {II:H II }}} \sim 1$ and $P_{\mathrm{H} \text { п:VH }} \sim P_{\mathrm{H} \text { п:Н }} \sim 0$. 
Thus we expect essentially all of the positrons that are either born in the inner bulge or diffuse into it from beyond, slow down and annihilate in the HII envelopes, making them the annihilation trap of the region.

\subsection{Middle Bulge $(0.5<R<1.5 \mathrm{kpc})$}

As Ferrière et al. (2007) have pointed out, "reliable observational information" in this region of the Galaxy is very sketchy and the gas distributions are very uncertain. The middle bulge from 0.5 to $1.5 \mathrm{kpc}$ is dominated by a tilted elliptical interstellar gas disk, observed primarily in $\mathrm{CO}$ and $\mathrm{H}_{\mathrm{I}}$ lines, with a hole in the middle of sufficient size to envelop the CMZ (Ferrière et al. 2007). They suggest that the most plausible disk model is that of Liszt \& Burton (1980) with a semimajor axis of $1600 \mathrm{pc}$ and a semiminor axis of $600 \mathrm{pc}$, and a central hole are $800 \mathrm{pc}$ and $260 \mathrm{pc}$, all tilted by $29^{\circ}$ relative to the Galactic plane. Thus, as can be seen in our Figure 1, this disk appears in a more face-on projection on the plane of the sky and extends $\sim 800 \mathrm{pc}$ above and below that plane. This is twice the nominal $400 \mathrm{pc}$ scale height of the overall Galactic thick disk modeled in the SPI/INTEGRAL analyses of the $511 \mathrm{keV}$ line emission (Weidenspointner et al. 2007, 2008a). Thus, we might expect that much of emission from positron annihilation in the tilted disk would not have been counted as part of the modeled thick planar disk fitted from the SPI/INTEGRAL data and would instead have been counted as part of their modeled extended spherical bulge from which it is essentially indistinguishable.

Here we model the interstellar phenomena of this outer portion of the tilted interstellar gas disk, again assuming that a hot tenuous plasma permeates the region, pressure equilibrium exists among all the interstellar phases, magnetic pressure is in equilibrium with gas thermal pressure, and the properties of the $\mathrm{HI}_{\mathrm{I}}$ and $\mathrm{H}_{2}$ regions are related to each other via the PDR scenario.

This disk contains an estimated $\sim 3.4 \times 10^{7} M_{\odot}$ of $\mathrm{H}_{2}$ and the corresponding disk-averaged gas density is $\left\langle n_{\mathrm{H}_{2}}\right\rangle \sim 4.8 \mathrm{~cm}^{-3}$ (Ferrière et al. 2007). In the $80 \%$ of the disk beyond $0.5 \mathrm{kpc}$, we assume that the $\mathrm{H}_{2}$ also resides in compact molecular clouds which are roughly similar to those in the CMZ with a nominal molecular core radius of $5 \mathrm{pc}$, but because of the lower external pressure, these clouds have a lower mean $\mathrm{H}_{2}$ density $n_{\mathrm{H}_{2}} \sim$ $1000 \mathrm{~cm}^{-3}$. Thus, the typical core mass, $M_{\mathrm{H}_{2} \text { core }}$ is $\sim 2.6 \times 10^{4}$ $M_{\odot}$, and the number of such cloud cores, $N_{\mathrm{H}_{2} \text { core }}$, is $\sim 1300$. For this $\mathrm{H}_{2}$ component we expect a cloud $\mathrm{H}_{2}$ filling factor in the outer part of the tilted disk, $f_{\mathrm{H}_{2}} \sim 4.8 / 1000 \sim 0.005$.

PDR shells surrounding molecular cloud cores must be sufficiently thick $\left(\sim 1.5 A_{v}\right)$ to shield the underlying $\mathrm{H}_{2}$ gas from dissociation via FUV absorption (Wolfire et al. 1993). A visual extinction, $A_{v} \sim 1.5$, corresponds to an $\mathrm{H}_{\mathrm{I}}$ column density $N(\mathrm{HI}) \sim 3 \times 10^{21} \mathrm{~cm}^{-2}$ (Tielens 2005). Thus to shield the tilted disk molecular cloud cores from dissociation, $n_{\mathrm{H}_{\mathrm{I}}} \Delta_{\mathrm{H}}$ of the overlying $\mathrm{H}_{\mathrm{I}}$ shells, $\sim 3 \times 10^{21} \mathrm{~cm}^{-2}$. If we assume that the nominal value of the HI density $n_{\mathrm{H}_{\mathrm{I}}} \sim 1000 \mathrm{~cm}^{-3}$, half the density of the $\mathrm{H}_{2}$ cores, then $\Delta r_{\mathrm{H}_{\mathrm{I}}} \sim 1$ pc. Thus, for the $\mathrm{HI}_{\mathrm{I}}$ interstellar component we expect a filling factor, $f_{\mathrm{H}_{\mathrm{I}}} \sim\left[\left(1+\Delta r_{\mathrm{H}_{\mathrm{I}}} / r_{\mathrm{H}_{2}}\right)^{3}-1\right] \times(4.8 / 1000) \sim 0.004$ in the disk.

These clouds, like those in the CMZ, are likely to be highly turbulent with the turbulent pressure balanced by the thermal pressure of the external hot medium (HM). Also as with the CMZ, we rely on analyses of X-ray observations to supply us with the expected HM pressure. In their analysis of the ROSAT $0.75 \mathrm{keV}$ all-sky survey, Almy et al. (2000) found that this region of the Galactic bulge is filled with a hot $\left(\leqslant 10^{7} \mathrm{~K}\right)$, tenuous $\left(\leqslant 10^{-2} \mathrm{~cm}^{-3}\right.$ ) plasma. However, this thermal HM pressure is significantly greater than the thermal pressure at which both cold H I and warm H I can coexist based on Wolfire et al. (2003). Thus, we expect that the cold $\mathrm{HI}$ is the only stable H I phase in the tilted disk region

Unlike the CMZ and inner tilted disk region, the outer tilted disk beyond around $0.5 \mathrm{kpc}$ does not appear to have any significant $\mathrm{H}$ II component. Although a population of molecular clouds exists in the tilted disk, no giant $\mathrm{H}$ II regions, standard tracers of massive star formation, have been resolved in this region (Smith et al. 1978). Moreover, no stars younger than 200 Myr have been found here by van Loon et al. (2003) in the mid-IR survey data from DENIS and ISOGAL. So we conclude that the stellar Lyman continuum flux and its resulting $\mathrm{H}$ II component are negligible in this region.

Therefore the filling factors in the tilted disk are $f_{\mathrm{H}_{2}} \sim$ $0.5 \%, f_{\mathrm{H}_{\mathrm{I}}} \sim 0.4 \%$, and $f_{\mathrm{HM}} \sim 99 \%$. The net cloud filling factor $\left(f_{c}=f_{\mathrm{H}_{\mathrm{I}}}+f_{\mathrm{H}_{2}}\right)$ implies a mean distance between neutral cloud centers $d_{c c} \sim 2 r_{c} / f_{c}^{1 / 3}$ of $\sim 60 \mathrm{pc}$, and a mean distance from a random point in the disk intercloud medium to a cloud surface of $\left(d_{c c} / 2\right)-\left(r_{c}+\Delta r_{\mathrm{H}}\right) \sim 25 \mathrm{pc}$.

We do not expect the SNIa and SNIp to be concentrated in this highly tilted disk which extends up to $\sim 800 \mathrm{pc}$ above the Galactic plane. For as we saw from Equation (3) and Figure 2, $55 \%$ of these $\mathrm{SNe}$ are in the stellar bulge population, distributed throughout the spherical shell from 0.5 to $1.5 \mathrm{kpc}$, and the other $45 \%$ are in the disk population, concentrated in the Galactic plane with a scale height of $\sim 0.18 \mathrm{kpc}$. Thus, the median distance from positron sources in the HM to the labyrinth of H I cloud envelopes in the disk is $\sim 0.5 \mathrm{kpc}$ for both populations. With the roughly vertical magnetic field in that region (e.g., Beck 2001), the positrons are born along flux tubes above and below the disk with a mean length $l_{B} \sim 1 \mathrm{kpc}$, twice the median value. We also expected that essentially all of the positrons are produced in the HM with the filling factor in the entire middle bulge, $f_{\mathrm{HM}} \sim 1$, and the other filling factors in the full middle bulge volume are $f_{\mathrm{H}_{2}} \sim 5 \times 10^{-5}$ and $f_{\mathrm{H}_{\mathrm{I}}} \sim 4 \times 10^{-5}$. Thus $Q_{\mathrm{HM}} \sim Q_{B m} \sim(0.37 \pm 0.09) \times 10^{43} \mathrm{e}^{+} \mathrm{s}^{-1}$, and $Q_{\mathrm{H}_{2}} \sim Q_{\mathrm{H}_{\mathrm{I}}} \sim 0$.

Since the HM filling factor is essentially unity, the subsequent positron propagation and annihilation in each of the phases of the outer titled disk is determined predominantly by the propagation fractions, which we estimate below, for the diffusion of positrons from the $\mathrm{HM}$ into the $\mathrm{H}$ i shells and on into $\mathrm{H}_{2}$ cloud cores, where they slow down and annihilate:

$$
\begin{aligned}
A_{\mathrm{HM}} & \sim Q_{\mathrm{HM}} P_{\mathrm{HM}: \mathrm{HM}}, \\
A_{\mathrm{HI}} & \sim Q_{\mathrm{HM}} P_{\mathrm{HM}: \mathrm{H}} P_{\mathrm{H} \mathrm{I}: \mathrm{HI}}, \\
A_{\mathrm{H}_{2}} & \sim Q_{\mathrm{HM}} P_{\mathrm{HM}: \mathrm{H}} P_{\mathrm{H} \mathrm{I}: \mathrm{H}_{2}} P_{\mathrm{H}_{2}: \mathrm{H}_{2}},
\end{aligned}
$$

where, like those discussed above, the propagation fractions $P$, subscripted HM:HM, $\mathrm{H}$ г:H I and $\mathrm{H}_{2}: \mathrm{H}_{2}$, are the fractions of the positron that slow down and annihilate in that phase, independent of whether they are produced in that phase or escaped into it from an adjacent phase, as given by the other $P$ combinations of escape from one phase to another adjacent phase. These fractions and annihilation rates are also listed in Table 2.

\subsubsection{Hot Medium (HM)}

Since the hot medium fills effectively all of the middle bulge, we expect that all of the positrons produced between 0.5 and 
$1.5 \mathrm{kpc}$ are born in the HM. Based on the analysis of Almy et al. (2000) at a Galactic planar radius of $\sim 1 \mathrm{kpc}$, which is the midpoint of this region, hot gas temperature $T_{\mathrm{HM}} \sim 5 \times 10^{6} \mathrm{~K}$, the density $n_{\mathrm{HM}} \sim 5 \times 10^{-3} \mathrm{~cm}^{-3}$. Thus the positron slowing down time $t_{\mathrm{sd}} \sim 9 \times 10^{6} \mathrm{yr}$ from Equation (5).

As with the $\mathrm{VH}$, we estimate the diffusion mean free path of the positrons in the HM as follows. We assume, as did Spergel \& Blitz (1992) for the CMZ, that the magnetic field pressure is in equilibrium with the gas pressure. Thus we expect $B_{o} \sim \sqrt{8 \pi p_{t h}} \sim 12 \mu \mathrm{G}$. As with the $\mathrm{CMZ}$ a plausible constraint on the nature of such turbulent flows is that the rate at which turbulent energy is dissipated is less than the rate at which the hot plasma cools. Almy et al. (2000) estimate the cooling time of this hot plasma, $t_{c} \sim 3 \times 10^{8} \mathrm{yr}$, but we again consider a conservative value of $\sim 5 \times 10^{7} \mathrm{yr}$. Approximating an outer scale, $l_{o} \sim 50 \mathrm{pc}$ for a hypothetical turbulent HM flow, this cooling time limits $\left(\overline{u^{2}}\right)^{1 / 2}$ to be less than $45 \mathrm{~km} \mathrm{~s}^{-1}$ for a thermal energy density, $\epsilon_{t h} \approx 1.5 \mathrm{p}_{t h} \approx 10^{-11} \mathrm{erg} \mathrm{cm}^{-3}$. Since the estimated Alfvén speed, $V_{a} \sim 310 \mathrm{~km} \mathrm{~s}^{-1}$, this limit on $\left(\overline{u^{2}}\right)^{1 / 2}$ corresponds to $\lambda_{\|} \sim 45$ pc by Equation (6). In the slowing down time, $t_{\mathrm{sd}}$ of $9 \times 10^{6} \mathrm{yr}$, HM positrons can diffuse a mean distance $l_{\text {sd }} \sim\left(2 \lambda_{\|} \bar{\beta} c t_{\text {sd }} / 3\right)^{1 / 2} \sim 7 \mathrm{kpc}$.

This distance is much larger than the mean flux tube length in the HM, $l_{B} \sim 1 \mathrm{kpc}$, along which the positrons are born above the labyrinth of $\mathrm{H}$ I covered clouds in the tilted disk. Thus as we saw with the $\mathrm{VH}$, we expect from Equation (8) that the fraction of positrons born in the $\mathrm{HM}$ and slowed down and annihilated there, $P_{\mathrm{HM}: \mathrm{HM}} \sim l_{B} / 2 l_{\mathrm{sd}} \sim 0.07$. As Monte Carlo simulations also suggested with the $\mathrm{VH}$, only a few percent of the positrons are likely to escape into the tenuous region above $1.5 \mathrm{kpc}$, while the bulk $(\sim 91 \%)$ diffuse into the cloudy labyrinths of both the tilted disk and the CMZ below. There due to the closeness of nearby clouds within these disks, essentially all of the positrons from the HM that enter these two labyrinths rapidly diffuse on into the turbulent outer envelopes of clouds where they slow down and annihilate.

We further estimate that the relative fractions of positrons born in the middle bulge that enter into the labyrinth of clouds in the outer tilted disk and annihilate in their H I envelopes, compared to those that escape down into the inner bulge and enter into the labyrinth of clouds in the $\mathrm{CMZ}$ and inner tilted disk and annihilate in their $\mathrm{H}$ II envelopes, are proportional to the relative surface areas of the two disks, or about $75 \%$ to $25 \%$, respectively. Thus, of the $91 \%$ of the positrons from the HM that enter these regions, we expect $P_{\mathrm{HM}: \mathrm{H}_{\text {II }}} \sim 0.22$, diffusing into the inner bulge, and the $\sim 0.69 \%$ remaining in the middle bulge are all expected to diffuse into the H I shells of clouds in the titled disk, and as we discuss below, essentially all slow down and annihilate in those shells, giving an effective $P_{\mathrm{HM}: \mathrm{H}_{\mathrm{I}}} \sim 0.69$ and $P_{\mathrm{HM}: \mathrm{H}_{2}} \sim 0$.

\subsubsection{Cold Medium (H I)}

As with the molecular clouds in the CMZ, we assume that only a fraction of the pressure is thermal in the tilted disk molecular cloud cores, so the mean temperature $T_{\mathrm{H}_{2}} \sim 20 \mathrm{~K}$ and density $n_{\mathrm{H}_{2}} \sim 1000 \mathrm{H}_{2} \mathrm{~cm}^{-3}$, although we assume they also have nominal radii of $\sim 5 \mathrm{pc}$. But because of the negligible filling factors of these cloud cores, no significant positron production should occur within them, and we show that essentially all of the positrons born elsewhere are expected to slow down and annihilate in the overlying $\mathrm{H}_{\mathrm{I}}$ envelopes before they can get into the $\mathrm{H}_{2}$ cores.
In the overlying $\mathrm{H}$ I envelopes between the cold $\mathrm{H}_{2}$ cloud cores and the hot HM plasma, we assume a mean density of $n_{\mathrm{H} \text { I }} \sim 1000 \mathrm{H} \mathrm{cm}^{-3}$ at a temperature $T_{\mathrm{H}_{\mathrm{I}}} \sim 100 \mathrm{~K}$, giving it thickness $\Delta r_{\mathrm{H}_{\mathrm{I}}} \sim 1 \mathrm{pc}$, as discussed above. Therefore the positron slowing down time in the $\mathrm{H} \mathrm{I}, t_{\mathrm{sd}}$ is $\sim 90 \mathrm{yr}$ from Equation (5), and since the MHD cascade is also damped by ion-neutral friction, the isotropic streaming positrons are distributed along a flux tube over a mean length $l_{\mathrm{sd}} \sim \bar{\beta} c t_{\mathrm{sd}} / 2 \sim 9 \mathrm{pc}$ in both directions in their slowing down time.

This is longer than the assumed nominal mean length of $l_{B}^{\prime} \sim(2 / 3)\left[(r+\Delta r)^{3}-r^{3}\right] /(r+\Delta r)^{2} \sim 1.7$ pc of unperturbed parallel flux tubes passing through a $\mathrm{H}$ i shell. But it is less than the mean meandering flux tube length $\left.l_{B} \sim\left(l_{B}^{\prime} / l_{o}\right) l_{B}^{\prime}\right) \sim 12 \mathrm{pc}$, expected for an turbulent scale $l_{o} \sim 0.25 \Delta r_{\mathrm{HI}} \sim 0.25 \mathrm{pc}$, if we assume the same shear flow scaling (Pope 2000), as discussed above.

Only a negligible fraction $(\sim 0.4 \%)$ of middle bulge positrons are expected to be born in the H I envelopes, but as we saw, $\sim 69 \%$ of all these positrons are expected to diffuse into the $\mathrm{H}$ I from the HM. As with the positrons that diffuse into the $\mathrm{H}$ II envelopes of CMZ clouds, these positrons are all effectively born within a scattering mean free path of the surface. Thus, since $l_{\text {sd }}<l_{B}$, we expect also that $\sim 0.5$ slow down an annihilate in the $\mathrm{HI}$, before they can penetrate into the $\mathrm{H}_{2}$ cores below, and the remainder escape back into the HM above. There, again because of the close proximity of the clouds, the escaping positrons quickly diffuse into another $\mathrm{H}$ I envelope, where the process is repeated, and soon virtually all are slowed down and annihilated in the $\mathrm{HI}$. Thus the net propagation fractions are

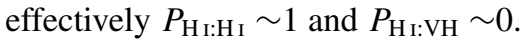

Although the density of the $\mathrm{H}$ I envelopes are quite uncertain, the expectation that they are thick enough to slow down and annihilate the positrons is rather robust. That results from the fact that the thickness of the $\mathrm{H}$ I and the slowing down length are both inversely proportional to the density, because the column depth of the $\mathrm{HI}$, which shields the $\mathrm{H}_{2}$ from dissociation, is fixed and so is the required column depth for the slowing down. Therefore, $l_{B}^{\prime}, l_{\mathrm{sd}}, l_{o}, l_{B}$, and $l_{B} / l_{\mathrm{sd}}$ all nominally scale proportionally.

Thus, we expect most ( $\sim 70 \%)$ of the positrons that are either born in the moddle bulge or diffuse into it from the outer bulge, slow down and annihilate in the $\mathrm{H}$ I envelopes of the clouds in the tilted disk, making them the annihilation trap of the region, as the H II envelopes are in the inner bulge.

As we discuss in the summary (Section 7), nearly all of the positrons that slow down in the $\mathrm{H}$ I envelopes in the middle bulge annihilate via fast positronium formation in charge exchange, producing broad $511 \mathrm{keV}$ line emission, while those that slow down in the Hit envelopes in the inner bulge annihilate predominantly via thermal radiative positronium formation, producing narrow $511 \mathrm{keV}$ line emission.

$$
\text { 4.3. Outer Bulge }(1.5<R<3.5 \mathrm{kpc})
$$

Based on the stellar disk distribution of SNIa and SNIp, as discussed above, we see from Figure 2 that $\sim 30 \%$ of all the stellar disk positrons and $\sim 22 \%$ of all the stellar bulge positrons produced by them should be made in the outer bulge between $1.5 \mathrm{kpc}$ and $3.5 \mathrm{kpc}$, and, as we now show, they become a major contributor to the modeled positron bulge component of the observed annihilation radiation within $1.5 \mathrm{kpc}$.

This region has been known for some time (Faber \& Gallagher 1976; Güsten 1989; Dehnen \& Binney 1998) to contain little H I, as traced by $21 \mathrm{~cm}$ radio line emission (Lockman 1984), and 
little $\mathrm{H}_{2}$, as traced by $2.6 \mathrm{~mm}$ line of CO (Sanders et al. 1984; Robinson et al. 1988). The lack of neutral gas results from a "high pressure galactic wind" (e.g., Blitz et al. 1993, Bregman 1980 ), driven primarily by stellar bulge $\mathrm{SNe}$ within $\sim 0.5 \mathrm{kpc}$, that blows out to at least $3 \mathrm{kpc}$, before it can recombine to form new neutral gas.

In this region, therefore, we consider only the hot plasma, with a mean density $n \sim 2 \times 10^{-3} \mathrm{~cm}^{-3}$ averaged over the scale height of $\sim 2 \mathrm{kpc}$, a conservative mean magnetic field strength, $B_{o} \sim 4 \mu \mathrm{G}$, an outer scale for MHD turbulence, $l_{o}$ $\sim 75 \mathrm{pc}$, based on simulations of SN-driven MHD turbulence (de Avillez \& Breitschwerdt 2004), and, finally, we assume that the rms strength of the turbulent magnetic field $\delta B / B_{o}$ $\sim 0.5$. From Equation (6), we then find that the scattering mean free path parallel to the mean magnetic field, $\lambda_{\|} \sim 14$ pc. Assuming that the bulge wind blows the magnetic field out radially, the mean length of a flux tube through the region is $l_{B} \sim 2 \mathrm{kpc}$.

In this ionized plasma the slowing down time $t_{\mathrm{sd}} \sim 23 \mathrm{Myr}$ from Equation (5). In that time the positrons diffuse both directions along a flux tube a mean length $l_{\mathrm{sd}} \sim\left(2 \lambda_{\|} \bar{\beta} c t_{\mathrm{sd}} / 3\right)^{1 / 2} \sim$ $6 \mathrm{kpc}$. Comparing this length with that of the flux tube, we expect from Equation (8) that the probability that positrons, born uniformly in the Bo, slow down and annihilate there is $\sim l_{B} / 2 l_{\text {sd }}$ $\sim 0.17$. But since the contribution from this region was also included in the model halo of Weidenspointner et al. (2008a), we include Bo there instead and take $1.5 \mathrm{kpc}$ as the inner boundary of the halo.

The remainder escape both into the bulge below and the disk and halo above. As we see from the distribution of the $\mathrm{SNe}$ in Figure 2, the $70 \%$ of the Bo SNe in the disk are rather uniformly distributed between the two boundaries and we assume that equal fractions escape up and down. However, since the remaining $30 \%$ from the bulge component are concentrated along an $l_{B}<1 \mathrm{kpc}$ close the inner boundary at $1.5 \mathrm{kpc}$, we expect that they escape primarily into the bulge and have a smaller chance of annihilating in the Bo. Thus, we estimate that the net fraction of Bo positrons annihilating there is $P_{B o: B o}$ $\sim 0.14$ and that escaping down into the middle bulge $P_{B o: B m}$ $\sim 0.57$.

As with the positrons produced in the middle bulge, we further expect that the $\sim 57 \%$ of the outer bulge positrons going down into the Bm from the Bo will also meet the same fates in the same proportions, mostly diffusing into the labyrinths of the tilted disk and the $\mathrm{CMZ}$, annihilating in the $\mathrm{HI}$ and $\mathrm{H}$ II envelopes of the molecular clouds. Thus, of all the positrons produced in the $\mathrm{Be}$ we expect $P_{B o: \mathrm{HI}} \sim 0.40$ and $P_{B o: H M} \sim 0.04$ in the middle bulge and $P_{B o: \mathrm{H}_{\text {II }}} \sim 0.13$ in the inner bulge.

Those positrons going beyond $3.5 \mathrm{kpc}$ are also expected to annihilate and not return, and within large uncertainties we assume that they have roughly equal likelihood of going into the halo or the disk. Thus $P_{B o: D o} \sim 0.14$, while, since the $\mathrm{Be}$ annihilation was also included in the halo, the net $P_{\text {Bo:H }} \sim 0.29$.

\subsection{Positron Bulge $(<1.5 \mathrm{kpc})$ Annihilation}

We now estimate the total positron annihilation rate within the $1.5 \mathrm{kpc}$ bulge using the contributions of the various phases and the propagation fractions within and between them, summarized in Table 2.

Using these fractions and combining Equations (9)-(13), the total annihilation rate in the positron bulge $<1.5 \mathrm{kpc}$ and its components are thus expected to be

$$
\begin{aligned}
A_{\mathrm{H} \text { II }} & \sim(0.46 \pm 0.08) \times 10^{43} \mathrm{e}^{+} \mathrm{s}^{-1}, \\
A_{\mathrm{HI}} & \sim(0.48 \pm 0.17) \times 10^{43} \mathrm{e}^{+} \mathrm{s}^{-1}, \\
A_{V H+H M} & \sim 0.06 \times 10^{43} \mathrm{e}^{+} \mathrm{s}^{-1}, \\
A_{B i} & \sim(0.47 \pm 0.08) \times 10^{43} \mathrm{e}^{+} \mathrm{s}^{-1}, \\
A_{B m} & \sim(0.53 \pm 0.13) \times 10^{43} \mathrm{e}^{+} \mathrm{s}^{-1}, \\
A_{B} & \sim(1.00 \pm 0.15) \times 10^{43} \mathrm{e}^{+} \mathrm{s}^{-1} .
\end{aligned}
$$

As we discuss in detail in the summary (Section 7), this is quite consistent with the best-fit bulge component annihilation rate from the SPI/INTEGRAL analyses (Weidenspointner et al. 2008a). Although we assumed large uncertainties of $\pm 50 \%$ in the estimated propagation fractions between interstellar gas phases, which produce comparably large uncertainties in the individual annihilation rates in each, their sums are more tightly constrained.

We also see (Table 2) that although the hot phase of the interstellar medium fills most of the bulge, because of propagation it is not the site of most of the annihilation. We find (Table 2 and Equation (13)) from the expected propagation and slowing down that $\sim 46 \% \pm 8 \%$ of the bulge positrons annihilate in the $\mathrm{H}$ II shells surrounding the molecular clouds in the highly ionizing radiation environment of the inner bulge $<0.5 \mathrm{kpc}$, via thermal positronium formation resulting in the narrow $(\sim 1.2 \mathrm{keV})$, $511 \mathrm{keV}$ line emission (Guessoum et al. 2005) from the bulge. We also see that $\sim 48 \% \pm 12 \%$ of the annihilation is expected to occur in the middle bulge, 0.5 to $1.5 \mathrm{kpc}$, in the neutral $\mathrm{H} \mathrm{I}$ shells surrounding the molecular clouds which lack significant $\mathrm{H}$ II shells, via in-flight positronium formation resulting in the broad ( $\sim 5.8 \mathrm{keV}), 511 \mathrm{keV}$ line emission from the bulge. As we discuss in detail below (Section 7), these two annihilation sites can account for the observed broad/narrow $511 \mathrm{keV}$ line ratio of $\sim 0.5$ (Churazov et al. 2005; Jean et al. 2006) in the positron bulge. We further point out that since these two components are expected to be essentially formed in separate, resolvable regions, spectral analyses of the two regions can test this prediction and further probe the structure of the bulge interstellar medium.

Only a fraction $P_{B o: B o} \sim 14 \%$ of the positrons are expected to slow down in the outer bulge, producing an annihilation rate $A_{B o} \sim 0.09 \times 10^{43} \mathrm{e}^{+} \mathrm{s}^{-1}$ in an essentially spherical shell contribution between 1.5 and $3.5 \mathrm{kpc}$. This is consistent with the effective limit of $<(0.2 \pm 0.2) \times 10^{43} \mathrm{e}^{+} \mathrm{s}^{-1}$ from that particular region, inferred from the best-fit modeling of the SPI/INTEGRAL data (Knödlseder et al. 2005). But since the contribution from that region has subsequently been included as part of the model halo in the analyses of Weidenspointner et al. (2008a), as discussed above, we include $A_{B o}$ in the halo annihilation instead. Even though the annihilation fraction in the outer bulge is small, its contribution to the observed $511 \mathrm{keV}$ line flux is still significant because of the high $\gamma_{511} / e^{+}$yield of $\sim 1.7$ expected (Jean et al. 2006) from positron annihilation in dusty hot plasma, as discussed above (Section 1.2).

In the next section we follow the remaining $\sim(0.16 \pm 0.10) \times$ $10^{43} \mathrm{e}^{+} \mathrm{s}^{-1}$ of the outer bulge positrons that escape beyond $3.5 \mathrm{kpc}$ into both the halo and the inner spiral arms that define the Galactic molecular ring of the interstellar disk. As we also show below (Section 5.4), the inner disk annihilation dominated by the these arms, which are viewed asymmetrically from our solar perspective, can fully account for the disk flux asymmetries (Weidenspointner et al. 2008b). 
Table 3

Assumed Properties of the Outer Bulge, Disk, and Halo

\begin{tabular}{|c|c|c|c|c|c|c|}
\hline \multirow{2}{*}{$\begin{array}{l}\text { Galactic } R \\
\text { Phase }\end{array}$} & \multirow{2}{*}{$\begin{array}{c}\text { Outer Bulge } \\
1.5-3.5 \mathrm{kpc} \\
\text { Bo }\end{array}$} & \multicolumn{4}{|c|}{$\begin{array}{c}\text { Disk } \\
>3.5 \mathrm{kpc}\end{array}$} & \multirow{2}{*}{$\begin{array}{c}\begin{array}{c}\text { Halo } \\
> \\
1.5 \mathrm{kpc}\end{array} \\
\mathrm{H}\end{array}$} \\
\hline & & HT & $\mathrm{HI}$ & WI & H II & \\
\hline Temperature $T \mathrm{~K}$ & $1 \times 10^{6}$ & $1 \times 10^{6}$ & 8000 & 8000 & 8000 & $1.6 \times 10^{6}$ \\
\hline Density $n \mathrm{H} \mathrm{cm}^{-3}$ & 0.002 & 0.002 & 0.13 & 0.30 & 2.6 & 0.002 \\
\hline Magnetic Field $B \mu \mathrm{G}$ & 4 & 4 & 4 & 4 & 4 & 4 \\
\hline Fluctuation $\delta B / B$ & $\sim 0.5$ & $\sim 0.5$ & $\ldots$ & $\sim 0.5$ & $\sim 1$ & $\sim 0.5$ \\
\hline Flux Tube $l_{B} \mathrm{pc}$ & $1000 \& 2000$ & $<1000$ & $\sim 3000$ & 160 & 85 & $>10000$ \\
\hline Max MHD Scale $l_{o} \mathrm{pc}$ & 75 & 75 & $\ldots$ & 10 & 2 & 75 \\
\hline$\lambda_{\|} \mathrm{pc}$ & 14 & 14 & $\ldots$ & 0.2 & 0.1 & 14 \\
\hline
\end{tabular}

\section{POSITRON PROPAGATION AND ANNIHILATION IN THE GALACTIC DISK ( $>3.5 \mathrm{KPC}$ )}

Having estimated the expected production, propagation and annihilation of positrons in the positron bulge $(<1.5 \mathrm{kpc})$ and in the outer bulge from 1.5 to $3.5 \mathrm{kpc}$, we now turn to the various components of the positron production, propagation, and annihilation in the outer disk $(D o)$ beyond $3.5 \mathrm{kpc}$.

As we have seen, the total expected positron production rate in this region consists of those born there, $Q_{D o}$, and those that diffuse into it from the outer bulge between 1.5 and $3.5 \mathrm{kpc}$, $Q_{B o} P_{B o: D o}$, we estimate

$$
\begin{aligned}
Q_{D o} & \sim(1.16 \pm 0.20) \times 10^{43} \mathrm{e}^{+} \mathrm{s}^{-1}, \\
Q_{B o} P_{B o: D o} & \sim(0.08 \pm 0.04) \times 10^{43} \mathrm{e}^{+} \mathrm{s}^{-1} .
\end{aligned}
$$

We again focus on the major phases of the interstellar medium, which fill essentially all of the outer disk volume in which essentially all of the $\mathrm{SNe}$ in this region occur, namely: the warm predominantly neutral $\mathrm{HI}$ medium, $(\mathrm{HI})$, the hot tenuous medium (HT), and the photoionized medium produced by $\mathrm{O}$ stars: the dense $\mathrm{H}_{\text {II }}$ regions ( $\mathrm{H}_{\mathrm{II}}$ ) and the diffuse warm ionized medium (WI). The dense ionized phases together with the molecular clouds, OB star associations and superbubbles are all concentrated along the spiral arms (e.g., Drimmel \& Spergel 2001; Stark \& Lee 2006), which dominate the structure of the outer disk. Their properties, discussed below, are summarized in Table 3.

We assume nominal average values of filling factors of these phases beyond $3.5 \mathrm{kpc}$ of $f_{\mathrm{HI}} \sim 0.50, f_{\mathrm{HT}} \sim 0.20, f_{\mathrm{H} \mathrm{II}} \sim 0.15$ and $f_{\mathrm{WI}} \sim 0.15$, based roughly on recent models (Ferrière 1998, 2001) of a thick disk of $\sim 400 \mathrm{pc}$ half thickness. There are large uncertainties in these numbers, but since they provide only an estimate of where the positrons are born, the uncertainties do not have a significant impact on where they eventually slow down and annihilate, for as we have seen elsewhere, that is dominated by their propagation. Although we do not consider independently the other interstellar phases of the disk, namely the molecular $\mathrm{H}_{2}$ and $\mathrm{H}_{\mathrm{I}}$ clouds, because of their very small filling factors $(<1 \%$ Ferrière 1998), we do consider their effects in the diffuse neutral medium $(\mathrm{HI})$.

As we saw from the integration of Equation (3) and Figure 2, $57 \%$ of the SNIa and SNIp in the disk occur $>3.5 \mathrm{kpc}$, producing $\sim(0.86 \pm 0.30) \times 10^{43} \mathrm{e}^{+} \mathrm{s}^{-1}$. We assume that they occur randomly in the various phases of the disk proportional to their filling fractions and that, as discussed above, $\sim 90 \%$ of the $\sim(0.3 \pm 0.1) \times 10^{43} \mathrm{e}^{+} \mathrm{s}^{-1}$, produced by ${ }^{26} \mathrm{Al}$ decay from massive stars in the disk, are born in the HT phase of hot superbubbles. Thus we expect that the positron production rates in each of the major phases of the disk beyond $3.5 \mathrm{kpc}$ are

$$
\begin{aligned}
& Q_{\mathrm{HT}}\sim(0.45 \pm 0.12)] \times 10^{43} \mathrm{e}^{+} \mathrm{s}^{-1}, \\
& Q_{\mathrm{HI}}\sim(0.45 \pm 0.15)] \times 10^{43} \mathrm{e}^{+} \mathrm{s}^{-1}, \\
&\left.Q_{\mathrm{H} \text { II }} \sim Q_{\mathrm{WI}} \sim(0.13 \pm 0.04)\right] \times 10^{43} \mathrm{e}^{+} \mathrm{s}^{-1} .
\end{aligned}
$$

From the positron production in these phases, we estimate the propagation fractions, listed in Table 4, and the resulting positron annihilation in the disk beyond $3.5 \mathrm{kpc}$ as follows:

$$
\begin{aligned}
& A_{\mathrm{HT}} \sim P_{\mathrm{HT}: \mathrm{HT}}\left(Q_{\mathrm{HT}}+Q_{\mathrm{H} \text { II }} P_{\mathrm{H} \text { п:НT }}+Q_{\mathrm{WI}} P_{\mathrm{WI}: \mathrm{HT}}\right), \\
& A_{\mathrm{HI}} \sim P_{\mathrm{HI}: \mathrm{HI}}\left(Q_{\mathrm{HI}}+Q_{\mathrm{HII}} P_{\mathrm{HII}} \mathrm{HI}_{1}+Q_{\mathrm{WI}} P_{\mathrm{WI}: \mathrm{HI}}\right), \\
& A_{\mathrm{H} \text { II }} \sim P_{\mathrm{H} \text { II:H II }}\left(Q_{\mathrm{H} \mathrm{II}}+Q_{\mathrm{HI}} P_{\mathrm{HI} \text { I:H II }}+Q_{\mathrm{WI}} P_{\mathrm{WI}: \mathrm{H} \mathrm{II}}\right. \\
& \left.+Q_{\mathrm{HT}} P_{\mathrm{HT}: \mathrm{H} \mathrm{II}}+Q_{B o} P_{B o: \mathrm{H} \mathrm{II}},\right) \\
& A_{\mathrm{WI}} \sim P_{\mathrm{WI}: \mathrm{WI}}\left(Q_{\mathrm{WI}}+Q_{\mathrm{HI}} P_{\mathrm{HI}: \mathrm{WI}}+Q_{\mathrm{HII}} P_{\mathrm{HII}}\right. \\
& \left.+Q_{\mathrm{HT}} P_{\mathrm{HT}: \mathrm{WI}}\right) \text {, }
\end{aligned}
$$

where, like those discussed above, the propagation fractions $P$, subscripted HT:HT, Hı:Hı, His:HII, and WI:WI, are the fractions of the positrons that are either produced in that phase or escape into it from an adjacent phase, that then slow down in and annihilate in that phase, while the other $P$ s are all combinations of escape from one phase to another adjacent phase. That excludes only direct escape from the HT into the $\mathrm{HI}$, or vise versa, because they are separated from one another by the H II and WI, and any return from the halo, which, we assume, is negligible.

Because of the large vertical scale height of the hot tenuous plasma in the large blown out superbubbles, annihilation there is also effectively part of the halo, not a resolvable component of the $\pm 400 \mathrm{pc}$ disk, so we add $A_{\mathrm{HT}}$ to $A_{H}$.

\subsection{Hot Tenuous Medium (HT)}

Ferrière (2001) found that the mean HT filling factor in the outer disk was a modest, $f_{\mathrm{HT}} \sim 0.2$. She also found that the HT was dominated by the contribution of superbubbles (e.g., Tomisaka \& Ikeuchi 1986; Heiles 1987, 1990; Mac Low \& McCray 1988) created by core-collapse $\mathrm{SNe}$, which in turn 
Table 4

Positron Propagation and Annihilation in the Outer Bulge, Disk, and Halo

\begin{tabular}{|c|c|c|c|c|c|c|}
\hline \multirow{2}{*}{$\begin{array}{l}\text { Galactic } R \\
\text { Phase } X\end{array}$} & \multirow{2}{*}{$\begin{array}{l}\text { Outer Bulge } \\
1.5-3.5 \mathrm{kpc} \\
\text { Bo }\end{array}$} & \multicolumn{4}{|c|}{$\begin{aligned} & \text { Disk } \\
> & 3.5 \mathrm{kpc}\end{aligned}$} & \multirow{2}{*}{$\begin{array}{c}\begin{array}{c}\text { Halo } \\
>1.5 \mathrm{kpc}\end{array} \\
\mathrm{H}\end{array}$} \\
\hline & & HT & $\mathrm{HI}$ & WI & H II & \\
\hline Filling Factor $f_{X}$ & 1 & $0.2 \pm 0.05$ & $0.5 \pm 0.1$ & $0.15 \pm 0.05$ & $0.15 \pm 0.05$ & 1 \\
\hline$Q_{X} 10^{43} e^{+} / \mathrm{s}$ & $0.56 \pm 0.14$ & $0.45 \pm 0.15$ & $0.45 \pm 0.15$ & $0.13 \pm 0.04$ & $0.13 \pm 0.04$ & 0 \\
\hline \multicolumn{7}{|l|}{ Propagation } \\
\hline$P_{B i / B m: X}$ & {$[\sim 0.04]$} & $\cdots$ & $\cdots$ & $\cdots$ & $\ldots$ & $\sim 0.04$ \\
\hline$P_{B o: X}$ & {$[\sim 0.14]$} & $\ldots$ & $\cdots$ & $\ldots$ & $0.14 \pm 0.07$ & $0.29 \pm 0.10$ \\
\hline$P_{\mathrm{HT}: \mathrm{X}}$ & $\ldots$ & {$[<\mathbf{0 . 0 8}]$} & $\ldots$ & $0.15 \pm 0.07$ & $0.15 \pm 0.07$ & $0.70 \pm 0.35$ \\
\hline$P_{\mathrm{HI}: X}$ & $\cdots$ & $\ldots$ & $\sim 0.02$ & $0.16 \pm 0.08$ & $0.16 \pm 0.08$ & $0.66 \pm 0.33$ \\
\hline$P_{\mathrm{WI}: \mathrm{X}}$ & $\cdots$ & $\sim 0$ & $\sim 0$ & $0.76 \pm 0.38$ & $0.05 \pm 0.02$ & $0.19 \pm 0.09$ \\
\hline$P_{\mathrm{H} \text { II: } X}$ & $\sim 0$ & $\sim 0$ & $\sim 0$ & $0.03 \pm 0.01$ & $0.85 \pm 0.42$ & $0.10 \pm 0.05$ \\
\hline$\underline{P_{H: X}}$ & $\sim 0$ & $\sim 0$ & $\sim 0$ & $\sim 0$ & $\sim 0$ & $\sim 1$ \\
\hline \multicolumn{7}{|l|}{ Annihilation } \\
\hline$Q P_{B i / B m: X} 10^{43} e^{+/ s}$ & {$[\sim 0.01]$} & $\cdots$ & $\cdots$ & $\cdots$ & $\ldots$ & $\sim 0.01$ \\
\hline$Q P_{B o: X}$ & {$[\sim 0.08]$} & $\ldots$ & $\cdots$ & $\ldots$ & $0.08 \pm 0.04$ & $0.16 \pm 0.08$ \\
\hline$Q P_{\mathrm{HT}: \mathrm{X}}$ & $\ldots$ & {$[<\mathbf{0 . 0 4}]$} & $\ldots$ & $0.07 \pm 0.03$ & $0.07 \pm 0.03$ & $0.31 \pm 0.15$ \\
\hline$Q P_{\mathrm{HI}: X}$ & $\cdots$ & $\ldots$ & $\sim 0.01$ & $0.07 \pm 0.03$ & $0.07 \pm 0.03$ & $0.30 \pm 0.15$ \\
\hline$Q P_{\mathrm{WI}: \mathrm{X}}$ & $\cdots$ & $\sim 0$ & $\sim 0$ & $\mathbf{0 . 1 0} \pm \mathbf{0 . 0 5}$ & $0.01 \pm 0.01$ & $0.02 \pm 0.01$ \\
\hline$Q P_{\mathrm{H}} \mathrm{II}: X$ & $\sim 0$ & $\sim 0$ & $\sim 0$ & $\sim 0$ & $0.11 \pm 0.05$ & $0.02 \pm 0.01$ \\
\hline$Q P_{H: X}$ & $\sim 0$ & $\sim 0$ & $\sim 0$ & $\sim 0$ & $\sim 0$ & $\sim 0$ \\
\hline$A 10^{43} e^{+} / \mathrm{s}$ & {$[\sim 0.09]$} & {$[\sim 0.04]$} & $\sim 0.01$ & $0.24 \pm 0.07$ & $0.34 \pm 0.08$ & $0.82 \pm 0.23$ \\
\hline
\end{tabular}

are correlated in space and time. We further found (Higdon \& Lingenfelter 2005$)$ that the majority $(\sim 85 \%)$ of core-collapse $\mathrm{SNe}$ (SNII \& SNIb/c) occur in superbubbles and an additional fraction $(\sim 20 \%)$ of the SNIa and SNIp occur there randomly with a probability equal to the superbubble filling factor.

Positron production in the superbubbles therefore comes both from the decay of ${ }^{26} \mathrm{Al}$ from the massive star WR winds and SNII, which create them, and from the decay of ${ }^{56} \mathrm{Co}$ and ${ }^{44} \mathrm{Ti}$ from the random SNIa and SNIp. Thus, as discussed above, the production in the HT is expected to be $Q_{\mathrm{HT}} \sim(0.45 \pm 0.12) \times$ $10^{43} \mathrm{e}^{+} \mathrm{s}^{-1}$, with $Q_{26} \sim(0.27 \pm 0.09) \times 10^{43} \mathrm{e}^{+} \mathrm{s}^{-1}$ from clustered massive stars and $Q_{\text {Ia+Ip }} \sim(0.18 \pm 0.04) \times 10^{43} \mathrm{e}^{+} \mathrm{s}^{-1}$ from the random SNIa and SNIp.

The typical properties of the hot medium (e.g., Ferrière 1998; Yan \& Lazarian 2004) are: a nominal temperature $T_{\mathrm{HM}} \sim$ $1 \times 10^{6} \mathrm{~K}$, a scale height averaged density, $n_{\mathrm{HM}} \sim 2 \times 10^{-3} \mathrm{~cm}^{-3}$, and a mean magnetic field, $B_{o} \sim 4 \mu \mathrm{G}$. As discussed in Section 4.1.1, the rate at which turbulence is dissipated must be less than the radiative rate at which the plasma cools. However, the cooling time of HM in outer Galaxy is very uncertain. Therefore we used $t_{\text {cool }} \sim 3 \times 10^{7} \mathrm{yr}$, the log average cooling time for a hot medium, whose evolution is dominated by time-dependent cloud evaporation following the prescription of McKee \& Ostriker (1977), and a cooling time from Tielens (2005) for the above values of $T_{\mathrm{HM}}$ and $n_{\mathrm{HM}}$. Using an outer scale, $l_{o} \sim 75 \mathrm{pc}$ (de Avillez \& Breitschwerdt 2004), the above cooling time limits $\left(\overline{u^{2}}\right)^{1 / 2}$ to be less than $40 \mathrm{~km} \mathrm{~s}^{-1}$. With $V_{a} \sim 80 \mathrm{~km} \mathrm{~s}^{-1}$ and the root-mean-square $\delta B / B_{o} \leqslant 0.5$, Equation (6) implies $\lambda_{\|} \geqslant$ $10 \mathrm{pc}$.

In this very tenuous plasma the positron slowing down time is $t_{\mathrm{sd}} \sim 23 \mathrm{Myr}$ from Equation (5) and the slowing down length $l_{\mathrm{sd}} \sim\left(2 \lambda_{\|} \bar{\beta} c t_{\mathrm{sd}} / 3\right)^{1 / 2}>6 \mathrm{kpc}$. Assuming a typical flux tube length of $l_{B}<1 \mathrm{kpc}$ in the superbubbles blowing out into the halo, the effective propagation fraction, $P_{\mathrm{HT}: \mathrm{HT}} \sim l_{B} / 2 l_{\mathrm{sd}}<0.08$. Since, as noted above, these scales are much larger than the disk SN distributions, there is no resolvable disk component of annihilation in the HT. Thus the $A_{\mathrm{HT}} \sim$ $P_{\mathrm{HT}: \mathrm{HT}} Q_{\mathrm{HT}}<0.04 \times 10^{43} \mathrm{e}^{+} \mathrm{s}^{-1}$ is included in the halo $A_{H}$.
As with the bulge wind dominated hot medium between 1.5 and $3.5 \mathrm{kpc}$, we again assume that the escaping positrons go into both the overlying halo and the photoionized, WI and $\mathrm{H}$ iI phases in the disk. For although the upper ends of these flux tubes are blown out into the overlying halo, so that the positrons could all escape, their roots feed into the surrounding H II and WI envelopes that separate the HT from the H I. Thus within large uncertainties, we also assume the nominal case where escape up into the halo or down into the surrounding photoionized gas is equally probable ( $\sim 46 \%$ each), and that of those entering the photoionized phases the WI and H II are also equally likely ( $\sim 23 \%$ each). As we show below, however, we expect that only $66 \%$ of the positrons that enter either the WI or the H II, slow down and annihilate before there, while the remainder escape into the halo, where they annihilate. Thus, we expect that the effective net propagation factors are $P_{\mathrm{HT}: \mathrm{WI}} \sim 0.15 \pm 0.07$, $P_{\mathrm{HT}: \mathrm{H}_{\text {II }}} \sim 0.15 \pm 0.07$, and $P_{\mathrm{HT}: \mathrm{H}} \sim 0.70 \pm 0.35$, including $P_{\mathrm{HT}: \mathrm{HT}}$.

\subsection{Warm Neutral Medium (H I)}

Studies by Wolfire et al. (2003) find that over most of the Galactic disk $>3.5 \mathrm{kpc}$ neutral $\mathrm{H}$ I gas resides in two stable, co-existing phases: a cold $(\sim 100 \mathrm{~K})$ neutral medium and a warm $\left(\leqslant 10^{4} \mathrm{~K}\right)$ neutral medium, but the filling factor of cold H I is negligible (Ferrière 1998). Further, Wolfire et al. found relative electron densities in the predominantly neutral $\mathrm{HI}, n_{\mathrm{HI}} / n_{i} \sim 1 / x_{e} \sim 50$ which greatly exceeds $f_{\text {crit }}$ of Equation (7), so turbulent Alfvénic cascades are damped by ion-neutral friction (e.g., Kulsrud \& Pearce 1969). Thus we expect the positrons to stream isotropically along magnetic flux tubes at $\bar{\beta} c / 2$.

The mean H I gas density seen by the positrons escaping into the interstellar medium from SNIa is $0.13 \mathrm{~cm}^{-3}$, using the H I gas density distribution from Ferrière (1998) applicable at $>3.5 \mathrm{kpc}$ and for our modeled SNIa and SNIp spatial distribution, Equation (3). Thus the slowing down time, $t_{\mathrm{sd}} \sim$ $7 \times 10^{5} \mathrm{yr}$ from Equation (5), and in that time they are spread 
both directions along a flux tube over a slowing down length $l_{\text {sd }} \sim \bar{\beta} c / 2 \sim 90 \mathrm{kpc}$.

We estimate the flux tube lengths in the $\mathrm{H}_{\mathrm{I}}$ in the Galactic disk from the mean separation between superbubbles, since are the primary agents for disrupting the interstellar magnetic field, which otherwise tends to lie in the plane of the disk. From an analysis of the Galactic distribution of OB associations by McKee \& Williams (1997), we estimate that the mean distance between superbubbles in the Galactic disk, $s \sim 0.42 e^{-R / H_{R}} \mathrm{kpc}$ as a function of galactocentric distance with $H_{R} \sim 3.5 \mathrm{kpc}$. Thus, the flux tube length $l_{B} \sim s$ may range from only $1.4 \mathrm{kpc}$ in the inner part of the warm disk at $R$ of $3.5 \mathrm{kpc}$ to about $4 \mathrm{kpc}$ at the solar radius, giving a mean $l_{B}$ of about $3 \mathrm{kpc}$. Since these distances are far less that the slowing down length, we would expect that only a very small fraction $\left(\sim l_{B} / 2 l_{\text {sd }} \sim 3 / 180 \sim 2 \%\right)$ of the positrons, born in the $\mathrm{H}$ I, slow down and annihilate there.

Thus, we expect that nearly all $(\sim 0.98)$ of the SNIa positrons, streaming along the magnetic flux tubes, escape from the $\mathrm{H}$ I into the overlying halo or the neighboring warm ionized $\mathrm{H}$ II and WI, going into the latter phases with equal likelihood. As we noted above, we expect that about $66 \%$ of the positrons entering either the WI or the $\mathrm{HI}$, slow down and annihilate there, while the remainder escape into the halo and annihilate. Therefore with large uncertainties, we take for the net factors, $P_{\mathrm{HI}: \mathrm{HI}} \sim 0.02$, and $P_{\mathrm{H} \text { I:WI }} \sim 0.16 \pm 0.08, P_{\mathrm{H} \text { I:H II }} \sim 0.16 \pm 0.08$, and $P_{\mathrm{H} \text { I:H }} \sim 0.66 \pm 0.33$.

\subsection{Photoionized Medium in the Galactic Disk}

OB associations ionize the interstellar medium, and create localized, high-density (H II) regions as well as an extended, diffuse warm ionized medium (WIM; e.g., McKee \& Williams 1997). Ionizing radiation from such clustered association stars, which are embedded initially in their parent molecular cloud, heat as well as ionize the surrounding dense molecular gas. Ultimately such ionized gas expands, streams away from the molecular cloud, evacuating large cavities, finally destroying the molecular cloud (Tenorio-Tagle 1979; Whitworth 1979). Thus OB associations create dense $\left(\geqslant 1000 \mathrm{~cm}^{-3}\right)$, compact $(\leqslant 1 \mathrm{pc})$ cores of photoionized gas, observable as luminous thermal radio sources and visible nebulae, but $\sim 2 / 3$ of the ionizing radiation emitted by massive association stars escapes these dense cores and create extended $(\geqslant 100 \mathrm{pc})$, more tenuous $\left(\leqslant 10 \mathrm{~cm}^{-3}\right.$ ) envelopes of photoionized gas (Anantharamaiah 1985; Heiles et al. 1996; McKee \& Williams 1997). Following McKee \& Williams, we model Galactic-disk H II regions as two components: the dense central cores surrounding the parent OB associations and the extended H II envelopes. As noted by McKee \& Williams, the tenuous H II envelopes completely dominate the $\mathrm{H}$ iI filling factor, and also about half the volume of an $\mathrm{H}$ II region is filled with a hot $\left(\geqslant 10^{6} \mathrm{~K}\right)$ tenuous, superbubble plasma.

Analyses of pulsar dispersion measures and diffuse, optical line emissions require the existence of a widespread (volume filling factor $\sim 20 \%)$, warm $(\sim 8000 \mathrm{~K})$, ionized medium (WI) of a modest density $\left(\sim 0.1 \mathrm{~cm}^{-3}\right)$ distributed throughout the Galactic disk and lower halo (Reynolds 1984, 1991; Tielens 2005). The extended WIM has a large scale height, $\sim 1 \mathrm{kpc}$, transverse the Galactic plane (Reynolds 1991; Taylor \& Cordes 1993), compared to $\sim 0.05 \mathrm{kpc}$, the scale height of O stars (Mihalas \& Binney 1981). However, a dilute O-star radiation field seems to explain many of WI line features (Mathis 1986 ; 2000). Thus it seems likely that a modest fraction, $\sim 0.15$, of Lyman-continuum radiation emitted by the planar
OB associations escapes from cloud-debris $\mathrm{H}$ II regions to ionize the high-latitude WI (e.g., Bregman \& Harrington 1986; Mathis 1986; Dove \& Shull 1994; Dove et al. 2000; Mathis 2000; Tielens 2005). Additionally, Slavin et al. (2000) found that the cooling of shock-heated gas, created by old supernova remnants located at high Galactic latitudes, creates a significant fraction of WI at these large kpc heights above the Galactic plane.

\subsubsection{Warm Ionized Medium (WI)}

Based on thermal pressure balance and the assumption that the WI is highly ionized, the mean electron density in the WI, $n_{e} \sim 0.26 \mathrm{~cm}^{-3}$ (Kulkarni \& Heiles 1987). The neutral fraction of the WI is not well constrained observationally by opticalline diagnostics, but neutral fractions $\sim 0.1$ seem likely (Tielens 2005). However, analyses of pulsar radio signals provide a stronger constraint on the WI ionization fraction. Radio-wave scatterings increase roughly linearly with dispersion measures $\sim 80 \mathrm{pc} \mathrm{cm}^{-3}$, a primary WIM tracer (Cordes et al. 1991). Thus Cordes et al. ascertained that the thermal electrons, which for create the pulsar dispersion measures, also generate corresponding radio scatterings. Since such radio scatterings are created by electron density fluctuations on spatial scales, $\sim 10^{9}-10^{11} \mathrm{~cm}$ (Cordes et al. 1991), significantly smaller than the mean free path for proton-H I collisions, $\sim 5 \times 10^{13} \mathrm{~cm}$ in unit-density media, means that efficient turbulent radio scattering exists in WI and, thus, $\mathrm{H}$ I fraction must be less than the critical value, Equation (7). Consequently, we assume that in WI $x_{e} \sim 0.9$.

A lower limit to the typical thickness of such WI regions is the mean free path of $13.6 \mathrm{eV}$ photons, $\left(n_{\mathrm{HI}} \bar{\alpha}_{\mathrm{HI}}\right)^{-1}$, where $\bar{\alpha}_{\mathrm{HI}} \sim 3 \times 10^{-19} \mathrm{~cm}^{2}$ (Tielens 2005) and the neutral H I density, $n_{\mathrm{HI}} \sim\left(1-x_{e}\right) n_{e} / x_{e}$. Taking a nominal value of $x_{e} \sim 0.9$ and $n \sim$ $n_{e} / x_{e} \sim 0.3 \mathrm{~cm}^{-3}$ gives a typical WI thickness, $\Delta l_{\mathrm{WI}} \sim 40 \mathrm{pc}$, and a positron slowing down time $t_{\mathrm{sd}} \sim 1.6 \times 10^{5} \mathrm{yr}$ from Equation (5).

Recent simulations (Esquivel et al. 2006) of the turbulent mixing generated by shear flow instabilities in the WI layers between the HT and HI suggest an outer turbulent scale $l_{o} \sim 10 \mathrm{pc}$ and $\left(\overline{u^{2}}\right)^{1 / 2} \sim 20 \mathrm{~km} \mathrm{~s}^{-1}$. Assuming $B_{o} \sim 4 \mu \mathrm{G}$, $\left(\delta B_{\perp}^{2}\right)^{1 / 2} / B_{o} \sim 1$, and $T_{\mathrm{WI}} \sim 8000 \mathrm{~K}$, we find from Equation (6) that $\lambda_{\|} \sim 0.2 \mathrm{pc}$. Thus in the slowing down time of $1.6 \times 10^{5} \mathrm{yr}$ we would expect the positrons to be distributed both directions along a flux tube over a distance $l_{\mathrm{sd}} \sim\left(2 \lambda_{\|} \bar{\beta} c t_{\mathrm{sd}} / 3\right)^{1 / 2} \sim 65 \mathrm{pc}$ in both directions along the flux tubes.

Again assuming that the mean length $l_{B}^{\prime}$ of nominally parallel flux tubes through these thick turbulent phases is equal to the typical thickness of the region, $\Delta l_{\mathrm{WI}} \sim 40 \mathrm{pc}$, we expect that with the turbulent scale of $\sim 10 \mathrm{pc}$, the mean meandering tube length $l_{B} \sim l_{B}^{\prime 2} / l_{o} \sim 40^{2} / 10 \sim 160 \mathrm{pc}$. The probability that those positrons initially born in the WI slow down and annihilate there before they escape into the neighboring $\mathrm{HI}$ and $\mathrm{HT}$ is $\sim l_{B} /\left(l_{B}+l_{\mathrm{sd}}\right) \sim 160 / 225 \sim 71 \%$. We expect that the escaping positrons enter into the $\mathrm{HI}_{\mathrm{I}}$ and $\mathrm{HT}$ with equal probability and end up annihilating in the WI, HII, or halo in the same relative proportions as those born in the H I and HT. Thus the effective propagation fractions for those born in the $\mathrm{WI}$ are $P_{\mathrm{WI}: \mathrm{WI}} \sim 0.76 \pm 0.38, P_{\mathrm{WI}: \mathrm{H} \text { II }} \sim 0.05 \pm 0.02$, and $P_{\mathrm{WI}: \mathrm{H}} \sim 0.19 \pm 0.09$.

On the other hand, the positrons, born in the H I and HT, that diffuse into the WI from are effectively reborn in the WI at their first scatter just inside the boundary, and since $l_{\text {sd }}<l_{B}$, we expect that $\sim 0.5$ of them slow down an annihilate in the WI, while the other half escape equally into the adjacent H I and HT. 
We also expect some of the escaping positrons to subsequently enter other WI phases and annihilate there, or in the $\mathrm{H}$ II or halo in the same relative proportions as those born in the $\mathrm{HI}$ and HT. Thus we expect that for the positrons that diffuse into the WI, their effective propagation fractions are $P_{\mathrm{WI}: \mathrm{WI}} \sim 0.58 \pm 0.29$, $P_{\mathrm{WI}: \mathrm{H} \mathrm{II}} \sim 0.08 \pm 0.04$, and $P_{\mathrm{WI}: \mathrm{H}} \sim 0.34 \pm 0.17$.

\subsubsection{H II Regions}

Although the direct positron production by $\mathrm{SNe}$ in the $\mathrm{H}$ II regions is modest because of their small filling factor, a large fraction of positrons made in the neighboring $\mathrm{HT}$ and $\mathrm{HI}$ escape into it. Thus, it becomes the major site of positron annihilation in the disk because of its high density $(\sim 10$ times that in the $\mathrm{HI}$ ), which reduces their slowing down time, so that most of the positrons slow down and annihilate before they can escape.

McKee \& Williams (1997) derived the Galactic volume filling factor of the $\mathrm{H}$ II regions as a function of galactocentric radius, $R$, assuming that these $\mathrm{H}$ II regions reproduced the observed Galactic plane dispersion measures. Further, they assumed the electron density is constant across the Galactic disk, and all the $R$-dependence resides in the filling factor, $f_{\mathrm{HII}}(R)$. They ascertained that $f_{\mathrm{HII}}(R)=f_{\mathrm{HII}}(0) e^{-R / H_{R}}$, where $H_{R}=3.5 \mathrm{kpc}$, and in such $\mathrm{H}_{\mathrm{II}}$ regions $n_{e}=2.6 \mathrm{~cm}^{-3}$, and $f_{\mathrm{HII}}(0)=0.13$. In these $\mathrm{H}$ II regions, the positrons slow down in $t_{\mathrm{sd}} \sim 1.7 \times 10^{4} \mathrm{yr}$ from Equation (5).

The typical thickness an $\mathrm{H}$ II envelope can be determined from the average dispersion measure contributed by the walls of a single large superbubble $\mathrm{H}$ II envelope, $\Delta \mathrm{DM} \sim 70 \mathrm{~cm}^{-3} \mathrm{pc}$ (McKee $\&$ Williams 1997). For a mean density $n_{e} \sim 2.6 \mathrm{~cm}^{-3}$, this corresponds to a typical wall thickness, $\Delta l_{\mathrm{H} \text { II }} \sim 0.5 \Delta \mathrm{DM} / n_{e} \sim 13$ pc. So, again assuming nominally parallel flux tubes, the mean length through one of these highly turbulent walls $l_{B}^{\prime}$ is also $\sim 13 \mathrm{pc}$. Although the nature of turbulent processes in the $\mathrm{H}$ II is poorly known, Haverkorn et al. (2004) have determined from the analysis of rotation measures that the outer scale of turbulent flows in disk $\mathrm{H}$ II regions, $l_{o}$, is relatively small, $\sim 2$ pc. From this, we then estimate the mean meandering tube length $l_{B} \sim l_{B}^{\prime 2} / l_{o} \sim 13^{2} / 2 \sim 85 \mathrm{pc}$.

To compare that with the expected slowing down length $l_{\text {sd }}$, we assume $B_{o} \sim 4 \mu \mathrm{G}$ and $T_{\mathrm{HII}} \sim 8000 \mathrm{~K}$, and estimate the diffusion mean free path in the $\mathrm{H}$ II using the cooling constraint, as we have done in other ionized phases. Following Tielens (2005), we find that the cooling time is short, $t_{\text {cool }} \sim 1.1 \times 10^{4} \mathrm{yr}$. So taking $l_{o} \sim 2 \mathrm{pc}$ and the constraint that the dissipation rate of the turbulence must be less than the cooling rate of the plasma, we find that the root-mean-square turbulent velocity, $\left(\overline{u^{2}}\right)^{1 / 2}<$ $23 \mathrm{~km} \mathrm{~s}^{-1}$, which is greater than the Alfvén speed in this plasma, $V_{a} \sim 5 \mathrm{~km} \mathrm{~s}^{-1}$, so $\left(\delta B^{2}\right)^{1 / 2} \sim B_{o}$, and from Equation (6) we thus find that $\lambda_{\|} \sim 0.1 \mathrm{pc}$.

In the slowing down time of $\sim 1.8 \times 10^{4} \mathrm{yr}$, we would thus expect the positrons to be distributed over a length $l_{\mathrm{sd}} \sim$ $\left(2 \lambda_{\|} \bar{\beta} c t_{\mathrm{sd}} / 3\right)^{1 / 2} \sim 15 \mathrm{pc}$ in both directions along a flux tube. Thus we expect from Equation (8) that $\sim l_{B} /\left(l_{B}+l_{\text {sd }}\right) \sim$ $85 /(85+15) \sim 85 \%$ of the positrons born in one of these $\mathrm{H}_{\text {II }}$ envelopes, would slow down and annihilate there before they escape into the neighboring H I and HT. We again assume that half of the escaping positrons go into each of these two phases, and that from there some diffuse into other $\mathrm{H}$ II and WI regions or into the halo and annihilate in the same proportion as those initially born in the H I and HT. Thus the effective propagation fractions for those born in the $\mathrm{H}_{\text {II }}$ are $P_{\mathrm{H} \text { ІІ Н ІІ }} \sim 0.87 \pm 0.43$, $P_{\mathrm{HII}: \mathrm{WI}} \sim 0.03 \pm 0.01$, and $P_{\mathrm{H} \text { II:H }} \sim 0.10 \pm 0.09$.
Again, however, most of the positrons in the $\mathrm{H}$ II were born in the $\mathrm{HI}$ and HT, and were reborn in the $\mathrm{H}$ II within a scattering mean free path of the boundary. As we have discussed above, with $l_{\text {sd }}<l_{B}$, we expect that $\sim 0.5$ of the positrons slow down an annihilate in the $\mathrm{H}$ II, and the other half escape equally into the adjacent Hi and HT. From there, we again expect them to eventually annihilate in the HII, WI, or halo in the same relative proportions as those born in the HI and HT. Thus, as with the WI discussed above, we expect that for the positrons that diffuse into the $\mathrm{H}_{\mathrm{II}}$, their effective propagation

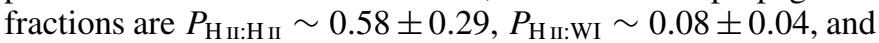
$P_{\mathrm{H} \text { II:H }} \sim 0.34 \pm 0.17$.

\subsection{Disk Annihilation Flux Asymmetry}

The asymmetry in the $511 \mathrm{keV}$ line flux (Weidenspointner et al. 2008b) from the inner disk provides further evidence of positron propagation and annihilation in the $\mathrm{H}$ II and WI. We show that this flux asymmetry can be fully accounted for by the apparent asymmetry of positron annihilation in the inner spiral arms, as viewed from our solar perspective, without any asymmetry in the positron production.

Modeling the inner Galactic disk in two components, Weidenspointner et al. (2008b) find a best-fit SPI/INTEGRAL 511 $\mathrm{keV}$ line flux of $(4.3 \pm 0.5) \times 10^{-4}$ photons $\mathrm{cm}^{-2} \mathrm{~s}^{-1}$ from the component between $-50^{\circ}<l<0^{\circ}$, compared to a flux of $(2.4 \pm 0.5) \times 10^{-4}$ photons $\mathrm{cm}^{-2} \mathrm{~s}^{-1}$ from that between $+50^{\circ}>l>0^{\circ}$. This amounts to an excess of $80 \% \pm 40 \%$ in the $511 \mathrm{keV}$ flux from negative longitudes compared to that from positive longitudes, and it amounts to an asymmetry of about $20 \% \pm 8 \%$ in the total observed disk flux (Weidenspointner et al. 2008a).

The asymmetry in the Galactic spiral arms, as viewed from our solar perspective, can be seen in Figure 4, showing the current mapping of the four spiral arms determined from the study by Vallée (2005a, 2005b). As summarized in Table 4, we expect that the positron annihilation in the disk occurs almost entirely in the warm ionized HII and WI phases, which are concentrated along the spiral arms (e.g., Drimmel \& Spergel 2001). Thus, we assume that equal fractions of the positrons produced at any particular radius propagate to and annihilate in the ionized gas in their nearest spiral.

We estimate the positron annihilation rate per unit length along each of the four arms using the radial distributions of the positron production surface density in the disk as a function of Galactocentric radius, $R$, given in Equation (3), multiplied by $\pi R d R / 2$ and by the disk annihilation/production fraction (Table 4). We further weight the annihilation distribution along the flux tubes by a unit normalized surface density distribution of interstellar gas, since positrons propagating along the flux tubes are more likely to encounter the dense ionized gas, where they annihilate, in denser regions. We also include the positrons escaping into the arms from the outer bulge. In accordance with observations (e.g., Drimmel \& Spergel 2001) that the Sagittarius arm is only half as dense and active as the others, we also reduce it accordingly.

At every point on each arm, we then multiply the differential annihilation rate by the $\gamma_{511} / e^{+}$ratio to determine the differential $511 \mathrm{keV}$ line luminosity and then divide by $4 \pi d_{\odot}^{2}$, where $d$ is the distance from the sun, to calculate the expected differential $511 \mathrm{keV}$ line flux at the earth. Finally, integrating along each arm out to $\pm 50^{\circ}$ Galactic longitude within $\pm 10^{\circ}$ latitude to compare with the SPI/INTEGRAL model, we find asymmetric $511 \mathrm{keV}$ line disk fluxes of $(3.8 \pm 1.0) \times 10^{-4}$ photons $\mathrm{cm}^{-2} \mathrm{~s}^{-1}$ from 


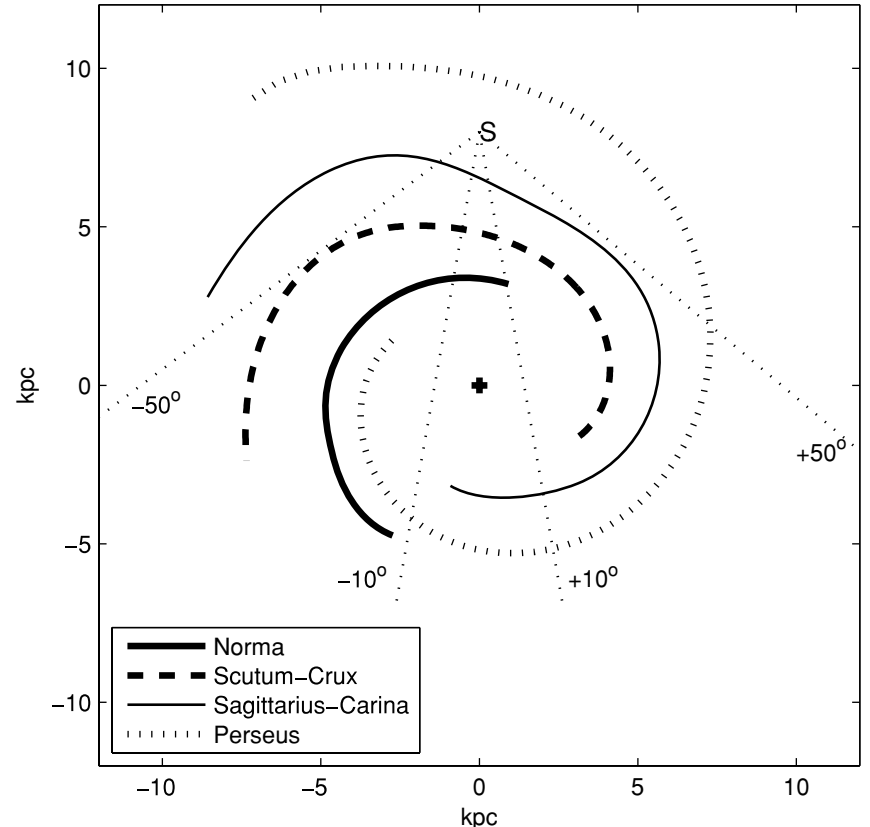

Figure 4. Galactic spiral arms, determined from the study by Vallee (2005a, 2005 b), showing the asymmetry, as viewed from our solar perspective, of the inner arms within $50^{\circ}$ to either side of the positron bulge $\left( \pm 10^{\circ}\right)$, measured by SPI/INTEGRAL. The asymmetry in the annihilation radiation from the Galactic disk is a product of the Norma arm dominating the negative longitudes due to its proximity to the inner region of the Galaxy, and the weakness of the SagittariusCarina arm due to its greater distance from the Galactic center from which the annihilation rate falls off exponentially from the Galactic center and its lower density/activity (Drimmel \& Spergel 2001) compared to the other 3 arms. We find $511 \mathrm{keV}$ line disk fluxes of $(3.8 \pm 1.0) \times 10^{-4}$ photons $\mathrm{cm}^{-2} \mathrm{~s}^{-1}$ from the negative longitudes, and $(2.3 \pm 0.6) \times 10^{-4}$ photons $\mathrm{cm}^{-2} \mathrm{~s}^{-1}$ from the positive longitudes, and a negative-to-positive longitude flux ratio of $1.6 \pm 0.4$. These values are quite consistent with the corresponding best fit (Weidenspointner et al. 2008b) SPI/INTEGRAL line fluxes.

the negative longitudes, and $(2.3 \pm 0.6) \times 10^{-4}$ photons $\mathrm{cm}^{-2}$ $\mathrm{s}^{-1}$ from the positive, and a negative-to-positive longitude flux ratio of $1.6 \pm 0.4$. These values are all quite consistent (Table 7) with the corresponding best-fit (Weidenspointner et al. 2008b) SPI/INTEGRAL line fluxes of $(4.3 \pm 0.5) \times 10^{-4}$ photons $\mathrm{cm}^{-2}$ $\mathrm{s}^{-1}$ and $(2.4 \pm 0.5) \times 10^{-4}$ photons $\mathrm{cm}^{-2} \mathrm{~s}^{-1}$, and a negativeto-positive flux ratio of $1.8 \pm 0.4$.

Thus we show that the observed (Weidenspointner et al. 2008b) SPI/INTEGRAL asymmetry in the $511 \mathrm{keV}$ line flux from the inner disk can be fully explained by the apparent asymmetry in the inner spiral arms as viewed from our solar perspective, which leads to different line-of-sight intensities, without any difference in the positron annihilation or production rates. This asymmetry in the annihilation radiation from the Galactic disk is a product of the Norma arm dominating the negative longitudes due to its proximity to the inner region of the Galaxy, and the weakness of the Sagittarius-Carina arm due to its greater distance from the Galactic center from which the annihilation rate falls off exponentially from the Galactic center and its lower density/activity (Drimmel \& Spergel 2001) compared to the other three arms.

\subsection{Disk Positron Annihilation $>3.5 \mathrm{kpc}$}

We now estimate the total outer disk annihilation rate from the contributions of the various phases and the propagation fraction within and between them. These are summarized in Table 4.

As we have discussed above, we see that more than half of the positrons, produced in the dominant phases of the interstellar medium in the disk, the $\mathrm{H}$ I and HT, escape into adjacent phases before they can slow down and annihilate. Only those produced in the HII and WI phases have a large chance of annihilating before they escape, and the rest either end up in the H II, the WI, or the halo. This result is quite robust, even allowing the large uncertainties in the relative likelihood of positrons following magnetic flux tubes that lead into these three annihilation traps.

Thus, we expect that the total annihilation rate in the disk beyond $1.5 \mathrm{kpc}$ is $A_{D} \sim(0.59 \pm 0.11) \times 10^{43} \mathrm{e}^{+} \mathrm{s}^{-1}$. As we discuss in detail in the summary (Section 7), this is quite consistent with the best-fit disk annihilation rate of inferred from the SPI/INTEGRAL analyses (Weidenspointner et al. 2007, 2008a).

\section{POSITRON PROPAGATION AND ANNIHILATION IN THE GALACTIC HALO}

Lastly, we consider the halo, which for comparison with the SPI/INTEGRAL analyses we take to lie beyond $1.5 \mathrm{kpc}$ from the Galactic center and more than $0.4 \mathrm{kpc}$ above the disk. Although it has no direct positron production by $\mathrm{SNe}$, a large fraction of positrons made in all the underlying phases of the bulge and disk can escape into it. Thus, it too becomes a major site of positron annihilation, because its pervasive hot tenuous plasma, coming primarily out of the outer bulge and disk superbubbles (e.g., Norman \& Ikeuchi 1989), may be turbulent enough to produce a scattering mean free path much smaller than its size, so that most of the positrons that enter it slow down and annihilate before they can escape on out of the Galaxy.

From the positron production in all of the regions and phases and their propagation fractions, discussed above, we estimate the positron escape into the halo and their annihilation there to be:

$$
\begin{aligned}
& A_{H} \sim P_{H: H}\left(Q_{B i} P_{B i: H}+Q_{B m} P_{B m: H}+Q_{B o} P_{B o: H}\right. \\
+ & \left.Q_{\mathrm{HT}} P_{\mathrm{HT}: \mathrm{H}}+Q_{\mathrm{HI}} P_{\mathrm{HI}: H}+Q_{\mathrm{WI}} P_{\mathrm{WI}: \mathrm{H}}+Q_{\mathrm{H} \mathrm{II}} P_{\mathrm{H} \mathrm{II}: H}\right) .
\end{aligned}
$$

Extensive observations of X-ray emission from the Galactic halo (e.g., Snowden et al. 1998; Pietz et al. 1998) have shown that hot tenuous plasma filling it is essentially indistinguishable from that in the outer bulge and superbubbles from which it is derived. Thus we assume a similar $T$ of $1 \times 10^{6} \mathrm{~K}$, a density of $2 \times 10^{-3} \mathrm{~cm}^{-3}$, and magnetic field, $B_{o} \sim 4 \mu \mathrm{G}$. This plasma has a scale height, $z_{X R}, \sim 4.4 \mathrm{kpc}$, and a radial scale length of $15 \mathrm{kpc}$. As discussed above for comparison with SPI/ INTEGRAL analyses, we define the halo as that region beyond a radius $1.5 \mathrm{kpc}$ and a $z>0.4 \mathrm{kpc}$, including within it the outer bulge.

The nature of turbulence in the halo is very uncertain, but we assume that a significant level of MHD turbulence can be maintained by erupting superbubbles. Using the parameters for that hot plasma, an outer turbulence scale, $l_{o} \sim 75 \mathrm{pc}$ and $\left(\overline{B_{\perp}^{2}}\right)^{1 / 2} / B_{o} \leqslant 0.5$, we also find the expected scattering mean free path parallel to the mean magnetic field, $\lambda_{\|} \sim 14 \mathrm{pc}$ from Equation (6).

Assuming a minimum flux tube escape length $l_{B} \sim 10 \mathrm{kpc}$, roughly 2 times the scale height, then the expected positron escape time $t_{\mathrm{esc}} \sim 200 \mathrm{Myr}$. By comparison the positron slowing down time, $t_{\mathrm{sd}} \sim 23 \mathrm{Myr}$ from Equation (5), and in that time positrons travel $l_{\mathrm{sd}} \sim\left(2 \bar{\beta} c t_{\mathrm{sd}} \lambda_{\|} / 3\right)^{1 / 2} \sim 7 \mathrm{kpc}$. Since all of the positrons enter the halo from below, rather than being born throughout, we expect that they will all slow down and annihilate well before they can escape, since $l_{\mathrm{sd}}<l_{B}$. Thus, for all of the positrons that enter the halo, we expect $P_{H: H} \sim 1$. Because the 
Table 5

Summary of Positron Production and Annihilation in Positron Bulge, Galactic Disk, and Halo

\begin{tabular}{|c|c|c|c|c|}
\hline Regions & $\begin{array}{c}\text { Production Rate } \\
\qquad 10^{43} \mathrm{e}^{+} / \mathrm{s} \\
\end{array}$ & $\begin{array}{c}\text { Annihilation Rate } \\
\qquad A 10^{43} \mathrm{e}^{+} / \mathrm{s}\end{array}$ & $\begin{array}{l}\text { Production } \\
Q / Q_{\text {Tot }} \% \\
\end{array}$ & $\begin{array}{c}\text { Annihilation } \\
A / A_{\text {Tot }} \% \\
\end{array}$ \\
\hline Bulge $<1.5 \mathrm{kpc}$ & $0.68 \pm 0.11$ & $1.00 \pm 0.19$ & $28 \pm 6$ & $41 \pm 8$ \\
\hline Bulge $1.5-3.5 \mathrm{kpc}$ & $0.56 \pm 0.14$ & {$[0.09]^{*}$} & $23 \pm 6$ & {$[4]^{*}$} \\
\hline Disk $>3.5 \mathrm{kpc}$ & $1.16 \pm 0.22$ & $0.59 \pm 0.11$ & $48 \pm 15$ & $25 \pm 5$ \\
\hline Halo $>1.5 \mathrm{kpc}$ & 0 & $0.82 \pm 0.21$ & 0 & $34 \pm 9$ \\
\hline Hot Plasma $\mathrm{H}^{+}$ & $1.66 \pm 0.24$ & $0.88 \pm 0.21$ & $69 \pm 13$ & $37 \pm 9$ \\
\hline Warm H II \& WI & $0.27 \pm 0.06$ & $1.04 \pm 0.14$ & $11 \pm 3$ & $43 \pm 6$ \\
\hline Warm Neutral H I & $0.45 \pm 0.15$ & $\sim 0.01$ & $19 \pm 7$ & $\sim 0$ \\
\hline Cold Neutral H I & $\sim 0$ & $0.48 \pm 0.17$ & $\sim 0$ & $20 \pm 7$ \\
\hline Molecular $\mathrm{H}_{2}$ & $\sim 0$ & $\sim 0$ & $\sim 0$ & $\sim 0$ \\
\hline \multicolumn{5}{|l|}{ Hot Plasma } \\
\hline Bulge $<1.5 \mathrm{kpc}$ & $0.65 \pm 0.11$ & $0.06 \pm 0.02$ & $27 \pm 6$ & $3 \pm 1$ \\
\hline Bulge $1.5-3.5 \mathrm{kpc}$ & $0.56 \pm 0.14$ & {$[0.09]^{*}$} & $23 \pm 6$ & {$[4]^{*}$} \\
\hline Disk $>3.5 \mathrm{kpc}$ & $0.45 \pm 0.12$ & {$[0.04]^{*}$} & $19 \pm 5$ & {$[2]^{*}$} \\
\hline Halo $>1.5 \mathrm{kpc}$ & 0 & $0.82 \pm 0.21$ & 0 & $34 \pm 9$ \\
\hline \multicolumn{5}{|l|}{ Warm H II \& WI } \\
\hline Bulge $<1.5 \mathrm{kpc}$ & $\sim 0.01$ & $0.46 \pm 0.08$ & $\sim 0$ & $19 \pm 4$ \\
\hline Disk $>3.5 \mathrm{kpc}$ & $0.26 \pm 0.06$ & $0.58 \pm 0.11$ & $11 \pm 3$ & $24 \pm 5$ \\
\hline \multicolumn{5}{|l|}{ Warm Neutral H I } \\
\hline \multicolumn{5}{|l|}{ Cold Neutral H I } \\
\hline Bulge $<1.5 \mathrm{kpc}$ & $\sim 0$ & $0.48 \pm 0.17$ & $\sim 0$ & $20 \pm 7$ \\
\hline
\end{tabular}

* Included in Halo

properties of the halo are assumed to be the same and those of the outer bulge and superbubble HT, which are the major sources of positrons that diffuse into it, there is no effective boundary between them and no "reflection" of those positrons from the halo. Moreover, as we discussed above, all positron annihilation in the outer bulge and superbubble HT is also included in the halo because of their large scale sizes.

From the estimates of the positrons entering the halo from the various underlying bulge and disk phases, summarized in Tables 4 and 5, we expect a total positron annihilation rate in the halo beyond $1.5 \mathrm{kpc}$ of $\sim(0.82 \pm 0.23) \times 10^{43} \mathrm{e}^{+} \mathrm{s}^{-1}$. As we show in Table 6 and discuss further in the summary, this annihilation rate gives an average $511 \mathrm{keV}$ line flux from the halo of $\sim(1.58 \pm 0.37) \times 10^{-3} \mathrm{~cm}^{-2} \mathrm{~s}^{-1}$, depending on how much refractory dust survives in the halo. This flux together with the bulge flux of $\sim(0.74 \pm 0.14) \times 10^{-3} \mathrm{~cm}^{-2} \mathrm{~s}^{-1}$ gives a combined bulge and halo flux of $\sim(2.32 \pm 0.40) \times 10^{-3}$ $\mathrm{cm}^{-2} \mathrm{~s}^{-1}$ (see Table 7), which is quite consistent with that of $\sim(2.14 \pm 0.12) \times 10^{-3} \mathrm{~cm}^{-2} \mathrm{~s}^{-1}$, determined from the SPI/ INTEGRAL best-fit flux model (Weidenspointner et al. 2008a).

As we discuss further in the summary, not only the $511 \mathrm{keV}$ line intensity but also the Galactic averages of both its broad/ narrow line flux ratio and the positronium fraction are very sensitive to the mean dust content of the hot halo plasma. For, as Jean et al. (2006) have shown and we list in Table 6, if the refractory elements are all in dust in the hot plasma, only $\sim 18 \%$ of the resulting positron annihilation occurs via positronium formation, and fully $\sim 90 \%$ of the $511 \mathrm{keV}$ line emission is narrow (FWHM $\sim 2 \mathrm{keV}$ ) while the remaining $10 \%$ is very broad (FWHM $\sim 11 \mathrm{keV}$ ). This gives a halo flux of $\sim(1.60 \pm 0.37) \times 10^{-3} \mathrm{~cm}^{-2} \mathrm{~s}^{-1}$ in the narrow line alone which in turn gives a combined bulge and halo flux of $\sim(2.34 \pm 0.40) \times 10^{-3} \mathrm{~cm}^{-2} \mathrm{~s}^{-1}$, which is likewise quite consistent with that from the SPI/INTEGRAL best fit above. Combined with disk flux, that would give a total Galactic bulge, disk, and halo flux of $\sim(3.25 \pm 0.42) \times 10^{-3} \mathrm{~cm}^{-2}$ $\mathrm{s}^{-1}$ which is also quite consistent with the combined bestfit flux of $\sim(2.87 \pm 0.25) \times 10^{-3} \mathrm{~cm}^{-2} \mathrm{~s}^{-1}$ from the SPI/ INTEGRAL analyses (Weidenspointner et al. 2008a). This also gives Galactic average values of the positronium fraction of only $\sim 0.66 \pm 0.12$ and the broad/narrow $511 \mathrm{keV}$ line flux ratio of only $\sim 0.17 \pm 0.04$, for which the best-fit SPI/INTEGRAL values have not yet been published.

If, on the other hand, the grains have all disintegrated, the halo flux would still be also as large, $\sim(1.41 \pm 0.31) \times 10^{-3} \mathrm{~cm}^{-2}$ $\mathrm{s}^{-1}$, but it would all be in the very wide $(\sim 11 \mathrm{keV}) 511 \mathrm{keV}$ line, so the Galactic average value of the broad/narrow $511 \mathrm{keV}$ line flux ratio would jump to $\sim 1.30 \pm 0.29$, and the positronium fraction would increase only slightly to $\sim 0.74 \pm 0.13$. The actual dust content of the halo undoubtedly lies in between and the SPI/INTEGRAL observations can provide a unique measure.

This is also consistent with that implied by the SPI/ INTEGRAL best-fit flux model of $A_{H} \sim(0.65 \pm 0.11) \times 10^{43}$ $\mathrm{e}^{+} \mathrm{s}^{-1}$ (Weidenspointner et al. 2008a), scaled to a galactocentric distance of $8 \mathrm{kpc}$ and $\left(e^{+} / \gamma_{511}\right) \sim 0.65 \pm 0.07$ expected (Jean et al. 2006) in the halo plasma, as discussed above.

\section{SUMMARY}

The recent analyses by Weidenspointner et al. (2007, 2008a) of the SPI/INTEGRAL measurements of the Galactic $511 \mathrm{keV}$ positron annihilation radiation luminosity suggest that the best fit to the observations consists of three basic components: (1) a spherical bulge of $\sim 1.5 \mathrm{kpc}$ radius, $(2)$ a thick $( \pm 400 \mathrm{pc})$ Galactic disk, and (3) a spherical halo extending beyond $1.5 \mathrm{kpc}$. Their most recent best-fit model (Weidenspointner et al. 2008a), scaled to a galactocentric distance of $8 \mathrm{kpc}$, gives $511 \mathrm{keV}$ line luminosities of $L_{B} \sim(0.57 \pm 0.08) \times 10^{43}$ photons s$^{-1}$ from the bulge, $L_{D} \sim(0.40 \pm 0.06) \times 10^{43}$ photons $\mathrm{s}^{-1}$ from the disk, and a net $L_{H} \sim(1.0 \pm 0.1) \times 10^{43}$ photons s$^{-1}$ from the 
Table 6

Galactic Positronium (Ps) Fraction and $511 \mathrm{keV}$ Line Luminosities and Fluxes

\begin{tabular}{|c|c|c|c|c|c|c|}
\hline \multirow[t]{2}{*}{ Properties } & \multicolumn{2}{|c|}{ Bulge } & \multicolumn{2}{|c|}{ Disk } & \multicolumn{2}{|c|}{ Halo } \\
\hline & $\begin{array}{c}\text { H II } \\
<0.5 \mathrm{kpc} \\
\end{array}$ & $\begin{array}{c}\text { H I } \\
0.5-1.5 \mathrm{kpc} \\
\end{array}$ & $\begin{array}{l}\text { H II \& WI } \\
>3.5 \mathrm{kpc}\end{array}$ & $\begin{array}{c}\mathrm{H} \mathrm{I} \\
>3.5 \mathrm{kpc} \\
\end{array}$ & $\begin{array}{c}\text { Grains } \\
>1.5 \mathrm{kpc} \\
\end{array}$ & $\begin{array}{c}\text { No Grains } \\
>1.5 \mathrm{kpc} \\
\end{array}$ \\
\hline Ps Fraction & 0.89 & 0.95 & 0.89 & 0.99 & 0.18 & 0.42 \\
\hline In-Flight Ps Fraction & 0.01 & 0.95 & 0.01 & 0.70 & 0 & 0 \\
\hline Thermal Ps Fraction & 0.88 & $\sim 0$ & 0.88 & 0.29 & 0.18 & 0.42 \\
\hline Direct Processes Fraction & 0.11 & 0.05 & 0.11 & 0.01 & 0.82 & 0.58 \\
\hline Broad $\gamma_{511} \%$ & 0 & 83 & 0 & 68 & 10 & 100 \\
\hline Narrow $\gamma_{511} \%$ & 100 & 17 & 100 & 32 & 90 & 0 \\
\hline \multicolumn{7}{|c|}{ Annihilation Rate $10^{43} \mathrm{e}^{+} \mathrm{s}^{-1}$} \\
\hline Total Positron & $0.46 \pm 0.08$ & $0.48 \pm 0.17$ & $0.58 \pm 0.11$ & $\sim 0.01$ & $0.82 \pm 0.21$ & $0.82 \pm 0.21$ \\
\hline Total Positronium & $0.41 \pm 0.08$ & $0.46 \pm 0.16$ & $0.52 \pm 0.10$ & $\sim 0.01$ & $0.15 \pm 0.04$ & $0.34 \pm 0.09$ \\
\hline In-Flight Positronium & 0 & $0.46 \pm 0.16$ & 0 & $\sim 0.01$ & 0 & 0 \\
\hline Thermal Positronium & $0.41 \pm 0.08$ & $\sim 0$ & $0.52 \pm 0.10$ & $\sim 0$ & $0.15 \pm 0.04$ & $0.34 \pm 0.09$ \\
\hline Mean $\mathrm{P}_{s}$ Fraction & \multicolumn{2}{|c|}{$0.93 \pm 0.08$} & \multicolumn{2}{|c|}{$0.90 \pm 0.11$} & \multicolumn{2}{|c|}{$\langle 0.3 \pm 0.12\rangle$} \\
\hline \multicolumn{7}{|l|}{ Luminosity $10^{43} \gamma \mathrm{s}^{-1}$} \\
\hline Ps $511 \mathrm{keV}$ Line & $0.20 \pm 0.04$ & $0.23 \pm 0.08$ & $0.26 \pm 0.05$ & $\sim 0.01$ & $0.07 \pm 0.02$ & $0.17 \pm 0.05$ \\
\hline Direct $511 \mathrm{keV}$ Line & $0.10 \pm 0.03$ & $0.04 \pm 0.02$ & $0.12 \pm 0.04$ & $\sim 0$ & $1.35 \pm 0.34$ & $0.96 \pm 0.24$ \\
\hline Total $511 \mathrm{keV}$ Line & $0.30 \pm 0.05$ & $0.27 \pm 0.08$ & $0.38 \pm 0.06$ & $\sim 0.01$ & $1.42 \pm 0.34$ & $1.13 \pm 0.25$ \\
\hline $3 \gamma$ Continuum & $0.88 \pm 0.18$ & $1.04 \pm 0.38$ & $1.17 \pm 0.23$ & $0.02 \pm 0.01$ & $0.33 \pm 0.08$ & $0.77 \pm 0.19$ \\
\hline Broad 511 keV Line & $\sim 0$ & $0.23 \pm 0.08$ & $\sim 0$ & $\sim 0.01$ & $0.14 \pm 0.04$ & $1.13 \pm 0.25$ \\
\hline Narrow $511 \mathrm{keV}$ Line & $0.30 \pm 0.05$ & $0.04 \pm 0.02$ & $0.38 \pm 0.06$ & $\sim 0$ & $1.28 \pm 0.30$ & 0 \\
\hline Broad 511 keV Line & \multicolumn{2}{|c|}{$0.23 \pm 0.08$} & \multicolumn{2}{|c|}{$\sim 0.01$} & \multicolumn{2}{|c|}{$\langle 0.64 \pm 0.15\rangle$} \\
\hline Narrow $511 \mathrm{keV}$ Line & \multicolumn{2}{|c|}{$0.34 \pm 0.05$} & \multicolumn{2}{|c|}{$0.38 \pm 0.06$} & \multicolumn{2}{|c|}{$\langle 0.64 \pm 0.15\rangle$} \\
\hline Broad/Narrow Line Ratio & \multicolumn{2}{|c|}{$0.68 \pm 0.26$} & \multicolumn{2}{|c|}{$\sim 0.03$} & \multicolumn{2}{|c|}{$\langle 1.0 \pm 0.2\rangle$} \\
\hline Broad/Narrow Line Ratio & \multirow{2}{*}{\multicolumn{4}{|c|}{$0.33 \pm 0.12$}} & & \\
\hline \multicolumn{4}{|l|}{ Flux $10^{-3} \gamma \mathrm{cm}^{2} \mathrm{~s}^{-1}$} & & & \\
\hline Total $511 \mathrm{keV}$ Line & $0.39 \pm 0.06$ & $0.35 \pm 0.12$ & $0.89 \pm 0.14$ & $\sim 0.02$ & $1.77 \pm 0.43$ & $1.41 \pm 0.31$ \\
\hline $3 \gamma$ Continuum & $1.15 \pm 0.24$ & $1.36 \pm 0.50$ & $2.73 \pm 0.65$ & $0.05 \pm 0.02$ & $0.41 \pm 0.11$ & $0.45 \pm 0.24$ \\
\hline Broad/Narrow Line Ratio & $\sim 0$ & $6 \pm 3$ & & & 0.11 & $\infty$ \\
\hline Total $511 \mathrm{keV}$ Line & \multicolumn{2}{|c|}{$0.74 \pm 0.14$} & \multicolumn{2}{|c|}{$0.91 \pm 0.14$} & \multicolumn{2}{|c|}{$\langle 1.59 \pm 0.37\rangle$} \\
\hline Broad 511 keV Line & \multicolumn{2}{|c|}{$0.30 \pm 0.10$} & \multicolumn{2}{|c|}{$\sim 0.02$} & \multicolumn{2}{|c|}{$\langle 0.79 \pm 0.19\rangle$} \\
\hline Narrow $511 \mathrm{keV}$ Line & 0.4 & .08 & 0.8 & & $\langle 0 . \tau$ & $19\rangle$ \\
\hline Broad/Narrow Line Ratio & 0.6 & .26 & & & & \\
\hline Broad $511 \mathrm{keV}$ Line & & 0.3 & 10 & & & \\
\hline Narrow $511 \mathrm{keV}$ Line & & 1.3 & 15 & & & \\
\hline Broad/Narrow Line Ratio & & 0.2 & 08 & & & \\
\hline
\end{tabular}

halo beyond the bulge, for a total Galactic $511 \mathrm{keV}$ luminosity $L \sim(2.0 \pm 0.2) \times 10^{43}$ photons s $^{-1}$.

This gives a positron annihilation luminosity bulge/disk ratio of $\sim 1.4 \pm 0.3$. But since this is a factor of 4 higher than the expected positron production ratio, inferred from the stellar bulge/disk ratio of the assumed Galactic SN sources, various exotic new sources have been invoked to explain the large discrepancy.

Here we have shown, however, that the measured $511 \mathrm{keV}$ luminosity ratio can be fully explained by positrons from the decay of radionuclei made by explosive nucleosynthesis in $\mathrm{SNe}$, if the propagation of these relativistic positrons in the various phases of the interstellar medium is taken into account, since these positrons must first slow down to energies $\leqslant 10 \mathrm{eV}$ before they can annihilate, and if the geometry of the sources is also considered. Moreover, as Jean et al. (2006) have shown, without propagation none of the proposed positron sources, new or old, can explain the two fundamental properties of the Galactic annihilation radiation: the fraction of the annihilation that occurs through positronium formation and the ratio of the broad/narrow components of the $511 \mathrm{keV}$ line.

\subsection{Positron Production}

First, we have shown, using recent estimates (Cappellaro et al. 1999) of the SNIa and SNIp rate in our Galaxy of $\sim 0.40 \pm$ $0.16 \mathrm{SN}$ per 100 years, that the positrons resulting from the $\beta^{+}$-decay chains of the radioactive nuclei, ${ }^{56} \mathrm{Ni},{ }^{44} \mathrm{Ti}$, and ${ }^{26} \mathrm{Al}$, produced in these $\mathrm{SNe}$ can fully account for the total Galactic positron production rate of $\sim(2.4 \pm 0.6) \times 10^{43} \mathrm{e}^{+} \mathrm{s}^{-1}$, implied by the best-fit SPI/INTEGRAL analyses (Weidenspointner et al. 2008a).

The total Galactic production rate of positrons from long lived (1.04 Myr meanlife) decay of ${ }^{26} \mathrm{Al}$ of $\sim(0.3 \pm 0.1) \times 10^{43}$ $\mathrm{e}^{+} \mathrm{s}^{-1}$, produced by massive stars, is determined directly from the SPI/INTEGRAL measurements (Diehl et al. 2006) of the Galactic luminosity of the $1809 \mathrm{keV}$ line emission which accompanies the decay. The Galactic positron production rate the ${ }^{44} \mathrm{Ti}$ decay chain $\left(89 \mathrm{yr}\right.$ meanlife) of $\sim(0.5 \pm 0.2) \times 10^{43}$ $\mathrm{e}^{+} \mathrm{s}^{-1}$ can be determined from the relative Galactic abundances (Lodders 2003) of ${ }^{44} \mathrm{Ca}$ and ${ }^{56} \mathrm{Fe}$, which are primarily produced by ${ }^{44} \mathrm{Ti}$ and ${ }^{56} \mathrm{Ni}$ decays, using the calculated SNIa rate and ${ }^{56} \mathrm{Ni}$ yield, assuming that these $\mathrm{SNe}$ presently produce about 
Table 7

Expected and Observed Galactic Positron Annihilation Radiation ${ }^{\mathrm{a}}$

\begin{tabular}{|c|c|c|c|}
\hline Properties & Expected & Observed & Reference \\
\hline Disk Component & $0.91 \pm 0.14$ & $0.94 \pm 0.16$ & $\quad, \quad, \quad \% \quad$, \\
\hline Halo \& Bulge Component & $2.32 \pm 0.40$ & $2.14 \pm 0.11$ & $" \quad, \quad " \quad$, \\
\hline Bulge Core $<0.5 \mathrm{kpc}$ & $0.39 \pm 0.10$ & $0.38 \pm 0.03$ & Weidenspointner et al. 2007 \\
\hline Bulge $<1.5 \mathrm{kpc}$ & $0.72 \pm 0.13$ & $0.79 \pm 0.07$ & $\quad, \quad, \quad, \quad$, \\
\hline Disk $-50^{\circ}<l<0^{\circ}$ & $\sim 0.38$ & $0.43 \pm 0.05$ & Weidenspointner et al. $2008 \mathrm{~b}$ \\
\hline Disk $+50^{\circ}>l>0^{\circ}$ & $\sim 0.23$ & $0.24 \pm 0.05$ & $" \quad, \quad$, \\
\hline Disk Ratio $\left(-50^{\circ}-0^{\circ}\right) /\left(+50^{\circ}-0^{\circ}\right)$ & $\sim 1.6$ & $1.8 \pm 0.4$ & $" \quad, \quad "$ \\
\hline \multicolumn{3}{|l|}{ Annihilation Rate } & \\
\hline Halo \& Bulge Combined & $1.95 \pm 0.28$ & $3.13\left[1.86^{*}\right] \pm 0.20$ & $"\left[*\right.$ adjusted $\left.\mathrm{e}^{+} / \gamma_{511}\right] "$ \\
\hline Bulge/Disk Component Ratio & $1.4 \pm 0.4$ & $1.4 \pm 0.3$ & $" \quad, \quad " \quad$, \\
\hline \multicolumn{4}{|l|}{ Positronium Fraction } \\
\hline Inner Galaxy & $0.92 \pm 0.08$ & $\begin{array}{l}0.94 \pm 0.06 \\
0.95 \pm 0.03 \\
0.92 \pm 0.09\end{array}$ & $\begin{array}{l}\text { Churazov et al. } 2005 \\
\text { Jean et al. } 2006 \\
\text { Weidenspointner et al. } 2006\end{array}$ \\
\hline Disk $>3.5 \mathrm{kpc}$ & $0.88 \pm 0.08$ & $-?-$ & $\cdots$ \\
\hline Halo $>1.5 \mathrm{kpc}$ & $0.65 \pm 0.07$ & $-?-$ & $\cdots$ \\
\hline \multicolumn{4}{|l|}{ Broad \& Narrow 511 keV Line } \\
\hline Bulge Warm Ionized Gas \% & $49 \pm 12$ & $51 \pm 3$ & Jean et al. 2006 \\
\hline Disk $>3.5 \mathrm{kpc}$ & $\sim 0.02$ & $-?-$ & $\cdots$ \\
\hline
\end{tabular}

Note. ${ }^{\text {a }}$ For a Solar Galactocentric distance of $8.5 \mathrm{kpc}$, assumed in the INTEGRAL analyses, instead of $8 \mathrm{kpc}$, assumed in this study.

half of the ${ }^{56} \mathrm{Fe}$ and core-collapse SNe produce the other half. The Galactic positron production from the much shorter lived (111.4 day meanlife) decay chain ${ }^{56} \mathrm{Ni}$, however, depends on an additional factor, since most of these decay positrons slow down and annihilate in the dense ejecta and only a fraction, $f_{56}$ survive into the interstellar medium. We showed that all of the additional positron production, amounting to $\sim 1.6 \times 10^{43}$ $\mathrm{e}^{+} \mathrm{s}^{-1}$, can be accounted for by the ${ }^{56} \mathrm{Ni}$ decay chain with a positron survival fraction in SNIa ejecta of $f_{56} \sim 5 \% \pm 2 \%$. This is the time-integrated mean of $\sim 5 \%$ calculated (Chan \& Lingenfelter 1993, Figure 3) from the standard deflagration models of SNIa (Nomoto et al. 1984, W7), assuming combed out magnetic fields, and it is also consistent with the mean fraction of $\sim 3.5 \% \pm 2 \%$ inferred (Milne et al. 1999) from SNIa light curves at late times.

Although the uncertainties in both the SNIa rate and the normalized survival fraction are large, $\sim 40 \%-50 \%$, they are necessarily anticorrelated. Therefore their product yields an uncertainty of only $\sim 25 \%$ in the production rates.

Using these positron production rates and the Galactic bulge and disk radial distributions of the SNIa, SNIp, and massive stars gave an expected positron production of $(0.31 \pm 0.07) \times 10^{43} \mathrm{e}^{+}$ $\mathrm{s}^{-1}$ in the inner bulge $<0.5 \mathrm{kpc},(0.37 \pm 0.09) \times 10^{43} \mathrm{e}^{+} \mathrm{s}^{-1}$ in the middle bulge from 0.5 to $1.5 \mathrm{kpc},(0.56 \pm 0.14) \times 10^{43} \mathrm{e}^{+} \mathrm{s}^{-1}$ in the outer bulge from 1.5 to $3.5 \mathrm{kpc}$, and $(1.16 \pm 0.22) \times 10^{43} \mathrm{e}^{+}$ $\mathrm{s}^{-1}$ in the disk beyond $3.5 \mathrm{kpc}$. For a positron annihilation bulge within $1.5 \mathrm{kpc}$ used in SPI/INTEGRAL observation analyses, that gave a positron production bulge/disk ratio of $0.4 \pm 0.1$ within $1.5 \mathrm{kpc}$. But, as we have shown, the expected annihilation ratio is very different, because the interstellar medium and the resulting positron propagation in these regions are also very different.

\subsection{Positron Propagation}

We have modeled the properties of the various regions and phases of the interstellar medium to determine the propagation of the positrons as they slowed down and annihilated. In ionized plasmas we assumed that diffusive propagation was controlled by resonant scattering of the relativistic positrons by MHD waves at their cyclotron radius. These waves are generated by the cascade of the larger scale magnetic turbulence down to sufficiently small scales which scatter the positrons and they diffuse along magnetic flux tubes. Such propagation has been observed in interplanetary plasmas with $\mathrm{MeV}$ electrons and we use a phenomenological model based on these studies. In neutral, or at least nearly neutral, phases of the interstellar medium, however, this cascade is damped by ion-neutral collisions, and the positrons are expected to stream along the magnetic flux tubes with an isotropic pitch angle distribution.

From these propagation processes, we have calculated the propagation, slowing down and annihilation of positrons formed in the various regions and phases of the interstellar medium described in Tables 1 and 3. From the properties of the medium in each phase, we determined the propagation mode, and calculated 
the diffusion mean free path in the undamped cascade mode, or the streaming velocity in the damped mode. From an estimate of the mean length of the magnetic flux tubes within a phase, compared to the tube length over which the positrons would propagate before they slowed down, we estimated the fraction of positrons expected to slow down and annihilate in that phase before they escape. We then estimated the relative fractions of the escaping positrons that go into each of the adjacent phases by simple geometric arguments. We have assumed large uncertainties of $\pm 50 \%$ in the estimated propagation fractions between phases, which produce comparably large uncertainties in the annihilation rates in each phase, although their sums within the bulge and disk are more tightly constrained. These propagation fractions between various components and the resulting distribution of their annihilation rates are presented in Tables 2 and 4.

\subsection{Positron Annihilation Rate and Flux}

The expected positron production and annihilation rates in the different regions and phases of the interstellar medium are summarized in Tables 5 and 6. Finally, the expected positron annihilation rates and $511 \mathrm{keV}$ line properties are compared with SPI/INTEGRAL analyses in Table 7.

We show that $\sim 52 \% \pm 7 \%$ of the all the Galactic positrons are expected to be produced within the bulge $<3.5 \mathrm{kpc}$, of which $\sim 28 \% \pm 6 \%$ are born within the positron bulge $<1.5 \mathrm{kpc}$. From the relative filling factors of the various gas and plasma phases in the bulge, we expect that essentially all of these positrons are born in the hot fully ionized plasma. From their propagation fractions we also expect that $\sim 80 \%$ of them diffuse into the warm $\mathrm{HII}$ and cold $\mathrm{Hi}$ envelopes of molecular clouds that lie within $1.5 \mathrm{kpc}$, where they slow down and annihilate, while the remaining $\sim 20 \%$ escape into the halo and disk beyond. This propagation thus results in a positron bulge annihilation rate $<1.5 \mathrm{kpc}$ of $A_{B} \sim(1.00 \pm 0.20) \times 10^{43} \mathrm{e}^{+} \mathrm{s}^{-1}$.

Of the positrons that are produced in the Galactic disk beyond $3.5 \mathrm{kpc}$, we expect from the relative filling factors that that about $70 \%$ of the disk positrons are born either in the ubiquitous warm neutral $\mathrm{H}$ I medium of the disk, or in the hot tenuous plasmas of superbubbles, in both of which the conditions are such that positrons rapidly stream or diffuse along magnetic flux tubes. Some segments of these flux tubes thread through the warm ionized gas and H II shells that separate the warm neutral gas from the hot tenuous plasmas, while other segments have been blown up into the halo by the hot expanding superbubbles (e.g., Parker 1979), and, since there is no preferred direction, the positrons diffusing or streaming along them should have roughly equal likelihood of either going into the warm denser ionized shells, where they mostly slow down and annihilate, or out into the halo. From the estimated propagation fractions, assuming large uncertainties, we find that nearly half of the positrons enter the ionized shells and annihilate in the disk, and that other half escape into the overlying halo, giving a total annihilation rate in the disk beyond $3.5 \mathrm{kpc}$ is $A_{D} \sim(0.59 \pm 0.11) \times 10^{43} \mathrm{e}^{+} \mathrm{s}^{-1}$.

So even though roughly equal numbers of positrons are born in the bulge within $3.5 \mathrm{kpc}$ and in the disk beyond, their annihilation rate is higher in the bulge than in the disk, because $\sim 80 \%$ of those born in the interstellar bulge slow down and annihilate in the cloud envelopes within $1.5 \mathrm{kpc}$, while only $\sim 51 \%$ of those born in the disk slow down and annihilate before they escape into the halo.

We now compare the spatial distribution of the Galactic positron annihilation radiation, expected from the $\beta^{+}$-decay chains of the radioactive nuclei, ${ }^{56} \mathrm{Ni},{ }^{44} \mathrm{Ti}$, and ${ }^{26} \mathrm{Al}$, produced in $\mathrm{SNe}$, with that observed by SPI/INTEGRAL.

The most direct comparison is through the $511 \mathrm{keV}$ line fluxes. From more than four years of SPI/INTEGRAL observations Weidenspointner et al. (2008a, model BD) fit the $511 \mathrm{keV}$ fluxes and luminosities to a combined bulge component within a spheroidal Gaussian (FWHM of $1.5 \mathrm{kpc}$ ) distribution and thick disk component. They found best-fit fluxes of $\sim(0.75 \pm 0.09) \times 10^{-3}$ photons $\mathrm{cm}^{-2} \mathrm{~s}^{-1}$ from the bulge and $\sim(0.94 \pm 0.16) \times 10^{-3}$ photons $\mathrm{cm}^{-2} \mathrm{~s}^{-1}$ from the disk. They also found a best-fit combined halo and bulge flux of $\sim(2.14 \pm 0.11) \times 10^{-3}$ photons $\mathrm{cm}^{-2} \mathrm{~s}^{-1}$.

For comparison we calculate in Table 5 the expected fluxes from the annihilation rates in the different regions and phases listed in Tables 2 and 4 using the $511 \mathrm{keV}$ photons yields per positron expected in those phases (Guessoum et al. 2005; Jean et al. 2006). Thus, we expect $511 \mathrm{keV}$ line fluxes of $\sim$ $(0.72 \pm 0.13) \times 10^{-3}$ photons $\mathrm{cm}^{-2} \mathrm{~s}^{-1}$ from the bulge, $\sim(1.08 \pm 0.16) \times 10^{-3}$ photons $\mathrm{cm}^{-2} \mathrm{~s}^{-1}$ from the disk, and $\sim(2.37 \pm 0.41) \times 10^{-3}$ photons $\mathrm{cm}^{-2} \mathrm{~s}^{-1}$ from the combined halo and bulge. These expected fluxes, as summarized in Table 7, are all in excellent agreement with the best-fit fluxes observed by SPI/INTEGRAL (Weidenspointner et al. 2008a).

We also calculated the expected $511 \mathrm{keV}$ fluxes from the inner bulge within $0.5 \mathrm{kpc}$ and the middle bulge between 0.5 and $1.5 \mathrm{kpc}, \sim(0.39 \pm 0.10) \times 10^{-3}$ and $\sim(0.33 \pm 0.07) \times 10^{-3}$ photons $\mathrm{cm}^{-2} \mathrm{~s}^{-1}$, respectively. As mentioned above, these boundaries were originally chosen for our calculations because these were the two regions modeled in the previous analysis by Weidenspointner et al. (2007) of the first two years of SPI/ INTEGRAL, where they found best-fit fluxes of $\sim(0.38 \pm$ $0.03) \times 10^{-3}$ and $\sim(0.41 \pm 0.06) \times 10^{-3}$ photons $\mathrm{cm}^{-2} \mathrm{~s}^{-1}$ from the same regions. These expected and observed values are also consistent within the $1 \sigma$ uncertainties.

We have compared with the earlier analysis, because the fluxes in the later Gaussian component analysis by (Weidenspointner et al. 2008a) overlapped in the inner volume which was also smaller than $0.5 \mathrm{kpc}$. The best-fit total fluxes from the analyses of (Weidenspointner et al. 2007) and (Weidenspointner et al. 2008a) within $1.5 \mathrm{kpc}$ are essentially the same, $\sim(0.79 \pm 0.07) \times 10^{-3}$ and $\sim(0.75 \pm 0.09) \times 10^{-3}$ photons $\mathrm{cm}^{-2} \mathrm{~s}^{-1}$, respectively.

We also compare the expected positron annihilation rates in the bulge, disk, and halo, and the corresponding bulge/disk ratio, with those determined by Weidenspointner et al. (2008a) from their best-fit SPI/INTEGRAL observations. Since they used a galactocentric distance of $8.5 \mathrm{kpc}$ rather than the $8 \mathrm{kpc}$, which we assumed, we scale our annihilation rates by $(8.5 / 8)^{2}$ for comparison. In addition, since $\sim 12 \%$ of their disk annihilation component lies within $1.5 \mathrm{kpc}$, we further modify our expected annihilation rates to compare with their model components. Thus, to compare with the Weidenspointner et al. (2008a) values, we calculate an equivalent SPI disk annihilation component ${ }_{S P I} A_{D} \sim A_{D}(8.5 / 8)^{2} /(1-0.12) \sim(0.76 \pm 0.13) \times 10^{43} \mathrm{e}^{+}$ $\mathrm{s}^{-1}$. This is in very good agreement with the SPI/INTEGRAL value of $(0.81 \pm 0.14) \times 10^{43} \mathrm{e}^{+} \mathrm{s}^{-1}$. Similarly, we calculate an equivalent SPI bulge annihilation component ${ }_{S P I} A_{B} \sim$ $\left(A_{B}-A_{D}[0.12 /(1-0.12)]\right)(8.5 / 8)^{2} \sim(1.04 \pm 0.21) \times 10^{43}$ $\mathrm{e}^{+} \mathrm{s}^{-1}$, which is also in very good agreement with the SPI/ INTEGRAL value of $(1.15 \pm 0.16) \times 10^{43} \mathrm{e}^{+} \mathrm{s}^{-1}$.

Thus, we expect an equivalent bulge/disk ratio of the annihilation rate ${ }_{S P I} A_{B} / S_{S P I} A_{D} \sim(1.04 \pm 0.21) /(0.76 \pm 0.13) \sim$ $1.37 \pm 0.37$, which is also in excellent agreement with the mea- 
sured value of $(1.15 \pm 0.16) /(0.81 \pm 0.14) \sim 1.42 \pm 0.32$ from the SPI/INTEGRAL data analyses of the best-fit bulge and disk components (Weidenspointner et al. 2008a). By comparison we also note, however, that the ratio of the total bulge annihilation with $1.5 \mathrm{kpc}$ compared to that in the disk beyond is $A_{B} / A_{D} \sim(1.00 \pm 0.19) /(0.59 \pm 0.11) \sim 1.69 \pm 0.45$. This is nonetheless consistent with the SPI/INTEGRAL best-fit model when the $12 \%$ disk component within $1.5 \mathrm{kpc}$ is subtracted from their disk component and added to that of the bulge, $\sim(0.94+0.08) /(0.66-0.08) \sim 1.79 \pm 0.38$.

We also showed that positron propagation and annihilation in the HII and WI gas along the spiral arms account for the asymmetry in the SPI/INTEGRAL $511 \mathrm{keV}$ line flux (Weidenspointner et al. 2008b) from modeled inner disk components within Galactic longitudes $\pm 50^{\circ}$ that show a best-fit excess of $80 \% \pm 40 \%$ in the $511 \mathrm{keV}$ line flux from the negative longitudes compared to that from the positive longitudes. As we have shown (Sections 5.4 and Table 7), this flux asymmetry can be fully explained by the apparent asymmetry in the inner spiral arms as viewed from our solar perspective, which leads to different line-of-sight intensities, without any actual asymmetry in the positron annihilation or production rates.

From the estimated fractions of the positrons entering the halo from the underlying bulge and disk, summarized in Table 4, we also compare the total expected positron annihilation rate in the halo with the SPI/INTEGRAL best-fit model of a combined bulge and halo component by Weidenspointner et al. (2008a). To do so we combine the expected bulge $(<1.5 \mathrm{kpc})$ and halo $(>1.5 \mathrm{kpc})$ annihilation rates from Table 5 , scaled to a galactocentric distance of $8.5 \mathrm{kpc}$, to give an equivalent SPI annihilation component, ${ }_{S P I} A_{H+B} \sim\left(A_{B}+\right.$ $\left.A_{H}\right)(8.5 / 8)^{2} \sim(1.95 \pm 0.28) \times 10^{43} \mathrm{e}^{+} \mathrm{s}^{-1}$. From their combined halo and bulge flux, discussed above, Weidenspointner et al. (2008a) estimate a significantly larger combined annihilation rate of $\sim(3.13 \pm 0.2) \times 10^{43} \mathrm{e}^{+} \mathrm{s}^{-1}$.

But they use a uniform $e^{+} / \gamma_{511} \sim 1.82$ to convert from observed the $511 \mathrm{keV}$ photons luminosity to an assumed positron annihilation rate. This conversion factor, however, was based on a positronium fraction of $\sim 97 \%$, determined by Jean et al. (2006) for warm gas in the inner Galaxy. Although this would be applicable to the bulge contribution, it is not applicable to the hot plasma of the halo contribution, for which Jean et al. (2006) estimate a positronium fraction of only about $18 \%$ to $42 \%$, depending on the refractory grain abundance, which corresponds to a $e^{+} / \gamma_{511} \sim 0.65 \pm 0.07$, as discussed above. Thus we use this much more appropriate conversion factor for flux contribution from the halo beyond the bulge, which from the best-fit fluxes amounts to roughly $65 \%$ of the combined halo and bulge flux. We estimate an annihilation rate of ${ }_{S P I} A_{H} \sim$ $(0.65 / 1.82)(3.13-1.15) \times 10^{43} \sim(0.71 \pm 0.26) \times 10^{43} \mathrm{e}^{+}$ $\mathrm{s}^{-1}$ just for the halo beyond the bulge, which, adding back the bulge contribution, gives an adjusted best-fit SPI/INTEGRAL model of $A_{H+B} \sim(1.86 \pm 0.26) \times 10^{43} \mathrm{e}^{+} \mathrm{s}^{-1}$. Those values would be in good agreement with the expected equivalent SPI annihilation components, ${ }_{S P I} A_{H} \sim(0.91 \pm 0.28) \times 10^{43}$ and ${ }_{S P I} A_{H+B} \sim(1.95 \pm 0.28) \times 10^{43} \mathrm{e}^{+} \mathrm{s}^{-1}$.

\subsection{Positronium Formation}

Significant propagation of the positrons also accounts for the other fundamental features of the Galactic annihilation radiation: (1) the high, observed fraction of positrons annihilating via positronium formation of $94 \% \pm 7 \%$, a weighted average of $94 \% \pm 6 \%$ (Churazov et al. 2005), $95 \% \pm 3 \%$ (Jean et al.
2006) and $92 \% \pm 9 \%$ (Weidenspointner et al. 2006), and (2) the observed ratio of broad $(\sim 5.8 \mathrm{keV})$ to narrow $(\sim 1.2 \mathrm{keV})$ $511 \mathrm{keV}$ line emission from the bulge of $\sim 0.5 \pm 0.2$ (Churazov et al. 2005; Jean et al. 2006), which gives a measure of the fraction of positronium that is formed by charge exchange with neutral $\mathrm{H}_{\mathrm{I}}$ in flight and results in broad line emission. For even though roughly $90 \%$ of the positrons in the bulge $(<1.5 \mathrm{kpc})$ are either born in, or diffuse into, the hot plasma that fills most of the bulge, hot plasma cannot be the site of most of the annihilation because neither the observed positronium fraction nor the broad to narrow line ratio is at all consistent with annihilation in that phase (Guessoum et al. 1991, 2005).

We show that the expected positron propagation, slowing down and annihilation in the bulge and disk quite naturally accounts for both of these observations. In particular, we expect (Table 5) that $(0.58 \pm 0.11) \times 10^{43} \mathrm{e}^{+} \mathrm{s}^{-1}$ annihilate in the warm ionized and $\mathrm{H}$ II phases of the disk, $(0.46 \pm 0.08) \times 10^{43}$ $\mathrm{e}^{+} \mathrm{s}^{-1}$ annihilate in the warm Hil phase of the bulge, and $(0.48 \pm 0.17) \times 10^{43} \mathrm{e}^{+} \mathrm{s}^{-1}$ annihilate in the cold neutral phase of the bulge. In these phases positronium is formed $\sim 89 \%$ and $\sim 95 \%$ of the time, respectively (Guessoum et al. 2005; Churazov et al. 2005; Jean et al. 2006), so we would expect (as outlined in Table 6) a combined positronium annihilation rate of $(1.40 \pm 0.74) \times 10^{43} \mathrm{e}^{+} \mathrm{s}^{-1}$ out of a total positron annihilation rate of $(1.53 \pm 0.81) \times 10^{43} \mathrm{e}^{+} \mathrm{s}^{-1}$ for a total Galactic positronium fraction $f_{P S} \sim 92 \% \pm 2 \%$.

We also expect (Table 6) that of the positronium formed in the warm ionized and $\mathrm{H}$ II phases $\sim 56 \%$ is in the disk and $\sim 44 \%$ is in the bulge, while only $\sim 2 \%$ of that formed in the neutral phases is in the disk and $\sim 98 \%$ is in the bulge. This gives an expected positronium fraction of $\sim 93 \% \pm 2 \%$ in the bulge and $\sim 90 \% \pm 2 \%$ in the disk (Table 6). The mean positronium fraction of $f_{P S} \sim 93 \% \pm 2 \%$ of the Galactic positron bulge within $1.5 \mathrm{kpc}$ is in very good agreement with the mean of $94 \% \pm$ $6 \%$ measured by SPI/INTEGRAL (Churazov et al. 2005; Jean et al. 2006; Weidenspointner et al. 2006) from the inner $\pm 20^{\circ}$ of the Galaxy.

\subsection{Broad/Narrow 511 keV Line Ratio}

We also consider the ratio of the broad and narrow components of the $511 \mathrm{kev}$ line fluxes, since the bulge and disk values are expected to be quite different (Table 6), and compare the expected values with those measured by SPI/INTEGRAL (Table 7).

In the warm ionized and $\mathrm{H}$ II phases, essentially all ( $99 \%)$ of the positronium is formed by radiative combination after the positrons are thermalized and its annihilation produces narrow ( $\sim 1.2 \mathrm{keV}$ FWHM), $2 \gamma 511 \mathrm{keV}$ emission $25 \%$ of the time and $3 \gamma$ continuum emission the rest of the time. In the cold neutral phases essentially all of the positronium is formed by charge exchange in flight producing the broad $(\sim 5.8 \mathrm{keV})$ Doppler shifted $511 \mathrm{keV}$ line, while in the warm neutral phase, which accounts for a negligible $\sim 2 \%$ of the neutral phase positronium formation, the fraction of in-flight formation is $\sim 70 \%$ for the typical weakly ionized $\left(x_{e} \sim 2 \%\right)$ gas considered here.

Thus, we would expect a total Galactic positron annihilation rate of $(0.47 \pm 0.12) \times 10^{43} \mathrm{e}^{+} \mathrm{s}^{-1}$ from positronium formed in-flight and an annihilation rate of $(0.93 \pm 0.11) \times 10^{43} \mathrm{e}^{+}$ $\mathrm{s}^{-1}$ from that formed thermally by radiative combination. With positronium producing two $511 \mathrm{keV}$ photons in $25 \%$ of its annihilations, this gives a broad $511 \mathrm{keV}$ line luminosity of $(0.24 \pm 0.06) \times 10^{43} \gamma \mathrm{s}^{-1}$ from in-flight annihilation and a narrow line luminosity of $(0.46 \pm 0.06) \times 10^{43} \gamma \mathrm{s}^{-1}$ from thermal 
annihilation. However, the residual direct thermal annihilation of $(0.12 \pm 0.06) \times 10^{43} \mathrm{e}^{+} \mathrm{s}^{-1}$ from nonpositronium processes also produces 2 narrow $511 \mathrm{keV}$ photons from every annihilation with a luminosity of $(0.26 \pm 0.12) \times 10^{43} \gamma \mathrm{s}^{-1}$, which gives a total narrow $511 \mathrm{keV}$ line luminosity of luminosity of $(0.72 \pm 0.14) \times 10^{43} \gamma \mathrm{s}^{-1}$.

We expect (Tables 6 and 7) that of the bulge and disk annihilation that $68 \%$ occurs in the warm ionized gas versus $32 \%$ in the neutral gas. Nearly all ( $98 \%)$ of the annihilation in the disk is in the warm ionized gas, while in the bulge we expect that $49 \% \pm 12 \%$ is in the ionized gas versus $51 \% \pm 14 \%$ in the neutral. The latter values are quite consistent with the SPI/INTEGRAL measurements from the bulge region, where Jean et al. (2006) find $51 \% \pm 3 \%$ in the ionized gas versus $49 \%$ $\pm 3 \%$ in the neutral.

We further expect (Table 6) that of the narrow line positronium formed in the warm ionized and $\mathrm{H}$ II phases $\sim 56 \%$ is in the disk and $\sim 44 \%$ is in the bulge, while again only $\sim 2 \%$ of that formed in the neutral phases is in the disk and $\sim 98 \%$ is in the bulge. In the bulge this yields a broad $511 \mathrm{keV}$ line luminosity of $(0.23 \pm 0.08) \times 10^{43} \gamma \mathrm{s}^{-1}$ from in-flight annihilation and a narrow line luminosity of $(0.34 \pm 0.06) \times 10^{43} \gamma \mathrm{s}^{-1}$ from thermal annihilation, for a broad/narrow line luminosity ratio of $0.68 \pm 0.26$. In the disk the broad line luminosity is only $\sim 0.01 \times 10^{43} \gamma \mathrm{s}^{-1}$ and the narrow line luminosity is $(0.38 \pm 0.06) \times 10^{43} \gamma \mathrm{s}^{-1}$, yielding a broad/narrow line luminosity ratio of only $\sim 0.03$, and the Galactic mean broad/ narrow line luminosity ratio from the combined bulge and disk is $0.33 \pm 12$.

However, the apparent ratio that would be seen from the Earth if the full disk were viewed is quite different, because of different relative weighting, since the observed fluxes from the bulge are equal to the bulge luminosities divided by $4 \pi R_{B}^{2} \sim 7.65 \times 10^{45} \mathrm{~cm}^{2}$, where $R_{B}$ is the distance to the Galactic center $\sim 8 \mathrm{kpc}$, and the observed fluxes from the disk are equal to the disk luminosities divided by only about $0.56 \times 4 \pi R_{B}^{2}$ (Weidenspointner et al. 2008a). Thus the broad line flux is $(0.30 \pm 0.08) \times 10^{-3} \gamma \mathrm{cm}^{-2} \mathrm{~s}^{-1}$ from the bulge and $(0.02 \pm 0.01) \times 10^{-3} \gamma \mathrm{cm}^{-2} \mathrm{~s}^{-1}$ from the disk, while the narrow line flux is $(0.44 \pm 0.08) \times 10^{-3} \gamma \mathrm{cm}^{-2} \mathrm{~s}^{-1}$ from the bulge and $(0.89 \pm 0.14) \times 10^{-3} \gamma \mathrm{cm}^{-2} \mathrm{~s}^{-1}$ from the disk. This gives a total observed broad $511 \mathrm{keV}$ line flux of $(0.32 \pm 0.11) \times 10^{-3}$ $\gamma \mathrm{cm}^{-2} \mathrm{~s}^{-1}$ and a narrow line flux of $(1.33 \pm 0.16) \times 10^{-3}$ $\gamma \mathrm{cm}^{-2} \mathrm{~s}^{-1}$, which gives an apparent broad/narrow line ratio of $0.24 \pm 0.08$. This ratio would be observed, however, only if the full disk were observed, and for smaller solid angles the apparent ratio would be expected to increase approaching the bulge value of $\sim 0.85$, when only the bulge is viewed. Thus, we expect the apparent ratio to be quite sensitive to the effective sky coverage, and we suggest that this angular dependence can test that prediction and further probe the nature and structure of the interstellar medium.

Indeed, analyses of SPI/INTEGRAL observations of the Galactic bulge by Jean et al. (2006) give a broad line flux of $(0.35 \pm 0.11) \times 10^{-3} \gamma \mathrm{cm}^{-2} \mathrm{~s}^{-1}$ and a narrow line flux of $(0.72 \pm 0.12) \times 10^{-3} \gamma \mathrm{cm}^{-2} \mathrm{~s}^{-1}$, which gives a broad/narrow line flux ratio of $0.49 \pm 0.17$. A separate analysis by Churazov et al. (2005) gives a broad line flux of $(0.24 \pm 0.10) \times 10^{-3} \gamma$ $\mathrm{cm}^{-2} \mathrm{~s}^{-1}$ and a narrow line flux of $(0.51 \pm 0.07) \times 10^{-3} \gamma \mathrm{cm}^{-2}$ $\mathrm{s}^{-1}$, which gives a broad/narrow line flux ratio of $0.47 \pm 0.20$. Considering the large uncertainties in both the measurements and the model value, the expected bulge ratio of $\sim 0.68 \pm 0.26$ is well within $1 \sigma$.
Moreover, within the positron bulge $(<1.5 \mathrm{kpc})$, we expect that the broad and narrow components of the $511 \mathrm{keV}$ line each essentially come from separate and potentially resolvable regions, and provide a further test. The narrow line emission is expected to come effectively all from the CMZ and inner tilted disk within $0.5 \mathrm{kpc}$, arising from thermal positronium and direct annihilation in the warm $\mathrm{H}$ II shells surrounding molecular clouds, ionized by FUV from central O stars. Essentially all of the broad line emission on the other hand is expected to come from the surrounding outer tilted disk between 0.5 and $1.5 \mathrm{kpc}$, arising from annihilation of positronium formed in flight in the cold neutral $\mathrm{H}_{\mathrm{I}}$ envelope of molecular clouds, where there is insufficient FUV to generate surrounding H II shells.

In particular, from the positron annihilation rates in Tables 2 and 6 , we expect from the $\mathrm{H}$ II shells within $0.5 \mathrm{kpc}$ of the Galactic center a narrow line flux of $(0.39 \pm 0.06) \times 10^{-3} \gamma$ $\mathrm{cm}^{-2} \mathrm{~s}^{-1}$ and no significant broad line flux, giving an expected broad/narrow line ratio of just $\sim 0$ within $0.5 \mathrm{kpc}$. Whereas from the $\mathrm{H}$ I gas in the surrounding tilted disk, we expect a broad line flux of $(0.30 \pm 0.10) \times 10^{-3} \gamma \mathrm{cm}^{-2} \mathrm{~s}^{-1}$, and a narrow line flux of $(0.05 \pm 0.03) \times 10^{-3} \gamma \mathrm{cm}^{-2} \mathrm{~s}^{-1}$ from direct thermal annihilation, giving an expected broad/narrow line ratio of $\sim 6 \pm 3$ between $0.5 \mathrm{kpc}$ and $1.5 \mathrm{kpc}$, which is nearly 10 times the combined full bulge average. Spectral analysis of the emission from these two regions should be able to test this prediction.

\subsection{Halo Contributions}

The $511 \mathrm{keV}$ line flux from the halo makes up nearly half of the total Galactic annihilation line flux measured by SPI/INTEGRAL (Weidenspointner et al. 2008a) and from our estimates of positron propagation we also expect that it accounts for a very similar $(\sim 49 \%)$ of the total line flux. Thus, as we discussed above, since this line intensity depends rather strongly on the mean dust content of the hot halo plasma, we expect that the Galactic averages of both the positronium fraction and the broad/narrow $511 \mathrm{keV}$ line flux ratio are also very sensitive to the amount of dust in the halo. For as Jean et al. (2006) have shown and we list in Table 6, if the refractory elements in the hot plasma are all in dust grains, only $\sim 18 \%$ of the positron annihilation occurs via positronium formation, and fully $\sim 90 \%$ of the $511 \mathrm{keV}$ line emission is narrow with a FWHM $\sim 2 \mathrm{keV}$, while the remaining $10 \%$ is very broad (FWHM $\sim 11 \mathrm{keV}$ ). This gives a halo flux of $\sim(1.60 \pm 0.37) \times 10^{-3} \mathrm{~cm}^{-2} \mathrm{~s}^{-1}$ in the narrow line alone. That in turn gives a combined bulge and halo flux of $\sim(2.34 \pm 0.40) \times 10^{-3} \mathrm{~cm}^{-2} \mathrm{~s}^{-1}$, which is quite consistent with that from the SPI/INTEGRAL best fit flux of $\sim(2.14 \pm 0.11) \times 10^{-3} \mathrm{~cm}^{-2} \mathrm{~s}^{-1}$ (Weidenspointner et al. 2008a), see Table 7. Combined with disk flux, that would give a total Galactic bulge, disk and halo flux of $\sim(3.25 \pm 0.42) \times 10^{-3}$ $\mathrm{cm}^{-2} \mathrm{~s}^{-1}$ which is also quite consistent with the combined bestfit flux of $\sim(2.87 \pm 0.25) \times 10^{-3} \mathrm{~cm}^{-2} \mathrm{~s}^{-1}$, from the SPI/ INTEGRAL analyses. This also gives Galactic average values of the positronium fraction of only $\sim 0.66 \pm 0.12$ and the broad/narrow $511 \mathrm{keV}$ line flux ratio of only $\sim 0.17 \pm 0.04$, for which the best-fit SPI/INTEGRAL values have not yet been published.

If, on the other hand, the grains have all disintegrated, the halo flux would still be almost as large, $\sim(1.41 \pm 0.31) \times 10^{-3} \mathrm{~cm}^{-2}$ $\mathrm{s}^{-1}$, but it would all be in the very wide $(\sim 11 \mathrm{keV}) 511 \mathrm{keV}$ line, so the Galactic average value of the broad/narrow $511 \mathrm{keV}$ line flux ratio would jump to $\sim 1.30 \pm 0.29$, and the positronium 
fraction would increase only slightly to $\sim 0.74 \pm 0.13$. The actual dust content of the halo undoubtedly lies in between and since we expect the broad/narrow $511 \mathrm{keV}$ line ratio from the halo beyond $1.5 \mathrm{kpc}$ to be extremely sensitive, ranging from $\sim 0.1$ to $\infty, \mathrm{SPI} / I N T E G R A L$ measurements of that ratio should provide a unique measure of the halo dust.

\section{CONCLUSION}

In conclusion, we find that roughly half of the Galactic positrons from $\mathrm{SN}$-generated radionuclei are produced in he bulge and inner disk within the stellar bulge of $R<3 \mathrm{kpc}$, and half in the stellar disk beyond that. Essentially all of the bulge positrons are produced in the very hot plasma, through which they diffuse with a calculated mean free path of only $1 \%$ of the size of the region. Therefore, before they can either slow down and annihilate in the plasma or escape beyond, most of them are stopped and annihilate in the very dense $(\sim 100$ $1000 \mathrm{H} \mathrm{cm}^{-1}$ ) warm outer shells of the molecular clouds that lie within $R<1.5 \mathrm{kpc}$. The disk positrons, on the other hand, are produced about equally in the warm Hi gas and the hot superbubbles, which blow out into the halo. We calculate that roughly half of the disk positrons, which either stream through the low density $\left(\sim 0.1 \mathrm{H} \mathrm{cm}^{-1}\right)$ neutral $\mathrm{H} \mathrm{I}$ at close to $c$, or diffuse through the hot superbubble plasma, escape into the overlying halo before they can stop and annihilate in the low density (0.3$3 \mathrm{H} \mathrm{cm}^{-1}$ ) photoionized outer shells of clouds and superbubbles in the disk. Thus, positron propagation easily explains the larger annihilation flux from the bulge. Moreover, it explains the high observed postronium fraction and narrow observed $511 \mathrm{keV}$ line width that both require annihilation predominantly in the warm ionized gas and not in the hot plasma in which nearly all of the positrons are produced, either in this or in other suggested sources.

We expect these conclusions to be quite robust, since they are based on (1) extensive observations of galactic SN rates and distributions, (2) observationally confirmed models of SN yields of radionuclei and their decay-positron survival fractions, (3) wide ranging observations of the properties and distributions of the various phases of the interstellar medium and magnetic fields, and (4) the widely used PDR models of molecular cloud shells. From these we have calculated the expected propagation of positrons in the various phases, assuming streaming in the neutral phases and diffusion in the ionized gas and plasma phases, using diffusion mean free paths in the latter phases calculated from a two-component, phenomenological model of anisotropic turbulence, which is consistent with observations of particle propagation in our best natural laboratory, the interplanetary medium.

Thus, as we show in Table 7, when positron propagation is considered, the positrons from the $\beta^{+}$-decay chains of the radioactive nuclei, ${ }^{56} \mathrm{Ni},{ }^{44} \mathrm{Ti}$, and ${ }^{26} \mathrm{Al}$, produced in Galactic $\mathrm{SNe}$, can fully account for all of the features of the diffuse Galactic $511 \mathrm{keV}$ and 3- $\gamma$ continuum annihilation radiation observed by SPI/INTEGRAL. We have also predicted additional measurable features that cannot only further test such an origin of the positrons but provide new information on the nature of the interstellar medium.

This work was supported by NASA's International Gamma-Ray Astrophysics Laboratory Science Program, grant NNG05GE70G.

\section{REFERENCES}

Almy, R. C., McCammon, D., Digel, S. W., Bronfman, L., \& May, J. 2000, ApJ, 545, 290

Anantharamaiah, K. R. 1985, JA\&A, 6, 203

Bale, S. D., Kellogg, P. J., Moser, F. S., Horbury, T. S., \& Reme, H. 2005, Phys. Rev. Lett., 94, 215002-1

Beck, R. 2001, Space Sci. Rev., 99, 243

Belcher, J. W., \& Davis, L. 1971, J. Geophys. Res., 76, 3534

Beresnyak, A., \& Lazarian, A. 2006, ApJ, 640, 185

Beresnyak, A., \& Lazarian, A. 2008, ApJ, 682, 1070

Berger, M. J., \& Seltzer, S. M. 1964, Tables of Energy Loses and Ranges of Electrons and Positrons (NASA SP-3012; Washington, DC: NASA)

Bieber, J. W., Wanner, W., \& Matthaeus, W. H. 1996, J. Geophys. Res., 101, 2511

Binney, J., \& Merrifield, M. 1998, Galactic Astronomy (Princeton NJ: Princeton Univ. Press)

Biskamp, D. 2003, Magnetohydrodynamic Turbulence (Cambridge: Cambridge Univ. Press), 99

Blinnikov, S., \& Sorokina, E. 2004, Ap\&SS, 290, 13

Blitz, L., et al. 1993, Nature, 361, 417

Bregman, J. 1980, ApJ, 237, 280

Bregman, J. N., \& Harrington, J. P. 1986, ApJ, 309, 833

Burger, J. J., Stephens, S. A., \& Swanenburg, B. N. 1970, Ap\&SS, 8, 20

Cappellaro, E., Evans, R., \& Turatto, M. 1999, A\&A, 351, 459

Carral, P., et al. 1994, ApJ, 423, 223

Chan, K.-W., \& Lingenfelter, R. E. 1993, ApJ, 405, 614

Cho, J., Lazarian, L., \& Vishniac, E. T. 2002, ApJ, 564, 291

Cho, J., \& Vishniac, E. T. 2000, ApJ, 539, 273

Churazov, E., Sunyaev, R., Sazonov, S., Revnivtsev, M., \& Varshalovich, D. 2005, MNRAS, 357, 377

Clayton, D. D. 1973, Nat Phys. Sci., 244, 137

Colgate, S. A. 1970, Ap\&SS, 8, 457

Colgate, S. A., Petschek, A. G., \& Kriese, J. T. 1980, ApJ, 237, L81

Cordes, J. M., Weisberg, J. M., Frail, D. A., Spangler, S. R., \& Ryan, M. 1991, Nature, 354, 121

Crutcher, R. M. 1999, ApJ, 520, 706

de Avillez, M. A., \& Breitschwerdt, D. 2004, in Proc. of the Magnetized Plasma in Galaxy Evolution, ed. K. T. Chyźy et al. (Kraków: Jagiellonian University), 66

Dehnen, W., \& Binney, J. 1998, MNRAS, 294, 429

Dermer, C. D., \& Murphy, R. J. 2001, Proc. 4th INTEGRAL Workshop (ESA SP-459; Noordwijk: ESA Publications Division), 115

Diehl, R., et al. 2006, Nature, 439, 45

Dove, J. B., \& Shull, J. M. 1994, ApJ, 423, 196

Dove, J. B., Shull, J. M., \& Ferrara, A. 2000, ApJ, 531, 846

Drimmel, R., \& Spergel, D. N. 2001, ApJ, 556, 181

Esquivel, A., Benjamin, R. A., Lazarian, A., Cho, J., \& Leitner, S. N. 2006, ApJ, 648, 1043

Faber, S. M., \& Gallagher, J. S. 1976, ApJ, 204, 365

Falceta-Goncalves, D., Lazarian, A., \& Kowal, G. 2008, ApJ, 679, 537

Felice, G. M., \& Kulsrud, R. M. 2001, ApJ, 553, 198

Ferreira, S. E. S., Potgieter, M. S., Burger, R. A., Heber, B., \& Fichtner, H. 2001a, J. Geophys. Res., 106, 24979

Ferreira, S. E. S., Potgieter, M. S., Burger, R. A., Heber, B., Fichtner, H., \& Lopate, C. 2001b, J. Geophys. Res., 106, 29313

Ferreira, S. E. S., Potgieter, M. S., Moeketsi, D. M., Heber, B., \& Fichtner, H. 2003, ApJ, 594, 552

Ferrière, K. 1998, ApJ, 497, 759

Ferrière, K. 2001, Rev. Mod. Phys., 73, 1031

Ferrière, K., Gillard, W., \& Jean, P. 2007, A\&A, 467, 611

Filippenko, A. V., et al. 1992, AJ, 104, 1543

Fredricks, R. W., \& Coroniti, F. V. 1976, J. Geophys. Res., 81, 5591

Freeman, K. C. 1985, in Proc. IAU Colloquium 88, Stellar Radial Velocities, ed. A. G. D. Philip, \& D. W. Latham (Schenectady: Davis Press), 223

Gillard, W., Jean, P., Marcowith, A., \& Ferrière, K. 2007, Proc. 6th INTEGRAL Workshop (ESA SP-622; Noordwijk: ESA Publications Division), 65

Goldreich, P., \& Sridhar, S. 1995, ApJ, 438, 763

Goldreich, P., \& Sridhar, S. 2006, ApJ, 640, L159

Greggio, L. 2005, A\&A, 441, 1055

Guessoum, N., Jean, P., \& Gillard, W. 2005, A\&A, 436, 171

Guessoum, N., Jean, P., \& Prantzos, N. 2006, A\&A, 457, 753

Guessoum, N., Ramaty, R., \& Lingenfelter, R. E. 1991, ApJ, 378, 170

Güsten, R. 1989, in IAU Symp. 136, The Center of the Galaxy, ed. M. Morris (Kluwer: Dordrecht), 89

Haverkorn, M., Gaensler, B. M., McClure-Griffiths, N. M., Dickey, J. M., \& Green, A. J. 2004, ApJ, 609, 776 
Heiles, C. 1987, ApJ, 354, 483

Heiles, C. 1990, ApJ, 315, 555

Heiles, C., Reach, W. T., \& Koo, B.-C. 1996, ApJ, 466, 191

Higdon, J. C. 1984, ApJ, 285, 109

Higdon, J. C., \& Lingenfelter, R. E. 2005, ApJ, 628, 738

Horbury, T. S., Balog, A., Forsyth, R. J., \& Smith, E. J. 1996, A\&A, 316, 333

Horbury, T. S., Forman, M., \& Oughton, S. 2005, Plasma Phys. Control. Fusion, 47, B703

Iroshnikov, P. S. 1964, SAJ, 7, 566

Jean, P., et al. 2006, A\&A, 445, 579

Johnson, W. N., Harnden, F. R., \& Haymes, R. C. 1972, ApJ, 172, 1

Jokipii, J. R. 1969, ApJ, 156, 1107

Kallenrode, M.-B. 1993, J. Geophys. Res., 98, 190327

Kennicutt, R. C. 2001, in ASP Conf. Ser. 231, Tetons 4: Galactic Structure, Stars, \& Interstellar Medium, ed. C. E. Woodward, M. D. Bicay, \& J. M. Shull (San Francisco, CA: ASP), 2

Knödlseder, J. 1999, ApJ, 510, 915

Knödlseder, J., et al. 2005, A\&A, 441, 513

Kolmogorov, A. 1941, Dokl. Akad. Nauk SSSR, 30, 301

Koyama, K. 1989, PASJ, 41, 664

Koyama, K., Maeda, Y., Sonobe, T., Takeshima, T., Tanaka, Y., \& Yamauchi, S. 1996, PASJ, 48, 249

Kraichnan, R. H. 1965, Phys. Fluids, 8, 1385

Kulkarni, S. R., \& Heiles, C. 1987, in Interstellar Processes, ed. D. J. Hollenbach, \& H. A. Thronson (Dordrecht: Reidel), 87

Kulsrud, R. M. 2005, Plasma Physics for Astrophysics (Princeton, NJ: Princeton Univ. Press), 346, 352

Kulsrud, R., \& Pearce, W. P. 1969, ApJ, 156, 445

Lazio, T. J., \& Cordes, J. M. 1998, ApJ, 505, 715

Leamon, R. J., Smith, C. W., Ness, N. F., Matthaeus, W. H., \& Wong, H. K. 1998, J. Geophys. Res., 103, 4775

Leventhal, M., MacCallum, C. J., \& Stang, P. D. 1979, ApJ, 225, 11

Li, H., et al. 2006, ApJ, 648, 340

Lingenfelter, R. E., Ramaty, R., \& Fisk, L. A. 1971, Astrophys. Lett., 8, 93

Liszt, H. S., \& Burton, W. B. 1980, ApJ, 236, 779

Lithwick, Y., \& Goldreich, P. 2001, ApJ, 562, 279

Lockman, F. J. 1984, ApJ, 23, 90

Lodders, K. 2003, ApJ, 591, 1220

Mac Low, M.-M., \& McCray, R. 1988, ApJ, 324, 776

Maron, J., \& Goldreich, P. 2001, ApJ, 554, 1175

Mason, J., Cattaneo, F., \& Boldyrev, S. 2006, Phys. Rev. Lett., 97, 255002

Mathis, J. S. 1986, ApJ, 301, 423

Mathis, J. S. 2000, ApJ, 544, 347

Matthaeus, W. H., Goldstein, M. L., \& Roberts, D. A. 1990, J. Geophys. Res., 95, 20673

McKee, C. F., \& Ostriker, J. P. 1977, ApJ, 218, 148

McKee, C. F., \& Williams, J. P. 1997, ApJ, 476, 144

Mezger, P. G., \& Pauls, T. 1979, in IAU Symp. 84, The Large Scale Characteristics of Galaxy, ed. W. B. Burton (Dordrecht: Reidel), 357

Mihalas, D., \& Binney, J. 1981, Galactic Astronomy (2nd ed.; San Francisco, CA: Freeman), 252

Milne, P. A., The, L.-S., \& Leising, M. D. 1999, ApJS, 124, 503

Mininni, P. D., \& Pouquet, A. 2007, Phys. Rev. Lett., 99, 254502

Miyazaki, A., \& Tsuboi, M. 2000, ApJ, 536, 357

Muno, M. P., et al. 2004, ApJ, 613, 326

Nomoto, K., Thielemann, F.-K., \& Yoko, K. 1984, ApJ, 286, 644

Norman, C. A., \& Ikeuchi, S. 1989, ApJ, 345, 372

Oka, T., Hasegawa, T., Hayashi, M., Handa, T., \& Sakamoto, S. 1998, ApJ, 493, 730

Osman, K. T., \& Horbury, T. S. 2007, ApJ, 654, L103

Oughton, S., Dmitruk, P., \& Matthaeus, W. H. 2004, Phys. Plasmas, 11, 2214

Oughton, S., Dmitruk, P., \& Matthaeus, W. H. 2006, Phys. Plasmas, 13, 042306

Oughton, S., \& Matthaeus, W. H. 2005, Nonlinear Proc. Geophys., 12, 299

Parker, E. N. 1979, in Cosmical Magnetic Fields (Oxford: Clarendon)
Perez, J. C., \& Boldyrev, S. 2008, ApJ, 672, L61

Pietz, J., Kerp, J., Kalberla, P. M. W., Burton, W. B., Hartmann, D., \& Mebold, U. 1998, A\&A, 332, 55

Pope, S. B. 2000, Turbulent Flows (Cambridge: Cambridge Univ. Press), 288

Prantzos, N. 2006, A\&A, 449, 869

Ramaty, R., \& Lingenfelter, R. E. 1979, Nature, 278, 127

Reynolds, R. J. 1984, ApJ, 282, 191

Reynolds, R. J. 1991, in IAU Symp. 144, The Interstellar Disk-Halo Connection in Galaxies, ed. H. Bloemen (Dordrecht: Kluwer), 67

Robinson, B. J., Manchester, R. N., Whiteoak, J. B., Otrupek, R. E., \& McCutcheon, W. H. 1988, A\&A, 193, 60

Rodriguez-Fernandez, N. J., \& Martin-Pintado, J. 2005, A\&A, 429, 938

Rodríguez-Fernández, N. J., et al. 2004, A\&A, 427, 217

Sanders, D. B., Solomon, P. M., \& Scoville, N. Z. 1984, ApJ, 276, 182

Schlickeiser, R. 2002, Cosmic Ray Astrophysics (Berlin: Springer)

Schekochihin, A. A., \& Cowley, S. C. 2007, in Magnetohydrodynamics: Historical Evolution and Trends, ed. S. Molokov, et al. (Berlin: Springer), 85 (arXiv:astro-ph/0507686v3)

Shebalin, J. V., Matthaeus, W. H., \& Montgomery, D. 1983, J. Plasma Phys., 29,525

Simien, F., \& de Vaucouleurs, G. 1986, ApJ, 302, 564

Sjouwerman, L. O., Habing, H. J., Lindqvist, M., van Langevelde, H. J., \& Winnberg, A. 1998, in The Central Parsecs of the Galaxy, ed. H. Falcke, V. Cotera, W. J. Duschl, F. Melia, \& M. J. Rieke (San Francisco, CA: ASP), 379

Slavin, J. D., McKee, C. F., \& Hollenbach, D. J. 2000, ApJ, 541, 218

Smith, L. F., Biermann, P., \& Mezger., P. G. 1978, A\&A, 66, 65

Snowden, S. L., Egger, R., Finkbeiner, D. P., Freyberg, M. J., \& Plucinsky, P. P. 1998, ApJ, 493, 715

Spergel, D. N., \& Blitz, L. 1992, Nature, 357, 665

Stark, A. A., \& Lee, Y. 2006, ApJ, 641, L113

Taylor, J. H., \& Cordes, J. M. 1993, ApJ, 411, 674

Tenorio-Tagle, G. 1979, A\&A, 71, 59

Teufel, A., \& Schlickeiser, R. 2002, A\&A, 393, 703

The, L.-S., et al. 2006, A\&A, 450, 1037

Tielens, A. G. G. M. 2005, The Physics and Chemistry of Interstellar Medium (Cambridge: Cambridge Univ. Press), 7

Tielens, A. G. G. M., \& Hollenbach, D. 1985, ApJ, 291, 722

Timmes, F. X., Woosley, S. E., \& Weaver, T. A. 1995, ApJS, 98, 617

Tomisaka, K., \& Ikeuchi, S. 1986, PASJ, 38, 697

Townsend, A. A. 1976, The Structure of Turblent Shear Flows (Cambridge: Cambridge Univ. Press), 188

Turatto, M., et al. 1996, MNRAS, 283, 1

Vallée, J. P. 2005a, ApJ, 619, 297

Vallée, J. P. 2005b, AJ, 130, 569

van den Bergh, S., \& Tammann, G. A. 1991, ARA\&A, 29, 363

van Loon, J. Th., et al. 2003, MNRAS, 338, 857

Weidenspointner, G., et al. 2006, A\&A, 450, 1013

Weidenspointner, G., et al. 2007, in Sixth INTEGRAL Workshop, ed. S. Grebenev, R. Sunyaev, \& C. Winkler (ESA SP-622; Noordwijk: ESA Publications Division), 25 (arXiv:astrop-ph/0702621)

Weidenspointner, G., et al. 2008a, New Astron. Rev., 52, 454

Weidenspointner, G., et al. 2008b, Nature, 451, 159

Whitworth, A. 1979, MNRAS, 186, 59

Wolfire, M. G., Hollenbach, D., \& Tielens, A. M. 1993, ApJ, 402, 195

Wolfire, M. G., Tielens, A. M., \& Hollenbach, D. 1990, ApJ, 358, 116

Wolfire, M. G., McKee, C. F., Hollenbach, D., \& Tielens, A. M. 2003, ApJ, 587, 278

Woosley, S. E., \& Pinto, P. A. 1988, in Nuclear Spectroscopy of Astrophysical Sources, ed. N. Gehrels, G. Share (New York: AIP), 98

Woosley, S. E., Taam, R. E., \& Weaver, T. A. 1986, ApJ, 301, 601

Woosley, S. E., \& Weaver, T. A. 1994, ApJ, 423, 371

Yamauchi, S., Kawada, M., Kyama, K., Kunieda, H., Tawara, Y., \& Hatsukade, I. 1990, ApJ, 365, 532

Yan, H., \& Lazarian, A. 2004, ApJ, 614, 757 